\title{
Structural Diversity of Perylenequinones is Driven by their Redox Behavior
}

Zeinab Y. Al Subeh, ${ }^{a}$ Amy L. Waldbusser, ${ }^{a}$ Huzefa A. Raja, ${ }^{a}$ Cedric J. Pearce, ${ }^{b}$ Kin Lok Ho, ${ }^{c}$

Michael J. Hall, ${ }^{\mathrm{c}}$ Michael R. Probert, ${ }^{\mathrm{c}}$ Nicholas H. Oberlies, ${ }^{* a}$ and Shabnam Hematian ${ }^{*}$ a

a Department of Chemistry and Biochemistry, University of North Carolina at Greensboro, Greensboro, North Carolina 27402, United States.

${ }^{\mathrm{b}}$ Mycosynthetix, Inc., Hillsborough, North Carolina 27278, United States.

${ }^{c}$ Chemistry, School of Natural and Environmental Sciences, Newcastle University, Newcastle upon Tyne, NE1 7RU, United Kingdom.

"To whom correspondence should be addressed: Dr. Nicholas Oberlies (email: nicholas_oberlies@uncg.edu) and Dr. Shabnam Hematian (email: $\underline{\text { s_hemati@uncg.edu). }}$ 
Figure S1. Structural similarity between hypocrellin (2) and cercosporin.

Figure S2. UPLC-(+)-HRESIMS spectrum of 7.

Figure S3. UV-vis spectrum of 7 in methanol at concentration of $0.0125 \mathrm{mg} / \mathrm{mL}$.

Table S1. NMR spectroscopic data for 7 in $\mathrm{CDCl}_{3}\left(700 \mathrm{MHz}\right.$ for ${ }^{1} \mathrm{H}$ and $175 \mathrm{MHz}$ for ${ }^{13} \mathrm{C}\left\{{ }^{1} \mathrm{H}\right\}, \delta$ in ppm).

Figure S4. ${ }^{1} \mathrm{H}$ - and ${ }^{13} \mathrm{C}\left\{{ }^{1} \mathrm{H}\right\}$-NMR spectra of $7\left[700 \mathrm{MHz}\right.$ for ${ }^{1} \mathrm{H}$ and $175 \mathrm{MHz}$ for $\left.{ }^{13} \mathrm{C}\left\{{ }^{1} \mathrm{H}\right\}, \mathrm{CDCl}_{3}\right]$.

Figure S5. Edited-HSQC NMR spectrum of 7 [700 MHz, $\left.\mathrm{CDCl}_{3}\right]$.

Figure S6. COSY NMR spectrum of $7\left[700 \mathrm{MHz}, \mathrm{CDCl}_{3}\right]$.

Figure S7. $\mathrm{HMBC}$ NMR spectrum of $7\left[700 \mathrm{MHz}, \mathrm{CDCl}_{3}\right]$.

Figure S8. Key HMBC correlations of 7.

Table S2. $E_{1 / 2}$ values for the cyclic voltammograms of 1-6 in MeCN with 100 $\mathrm{mM}$ of $\left[(n \mathrm{Bu})_{4} \mathrm{~N}\right]\left[\mathrm{PF}_{6}\right]$ as the supporting electrolyte.

Table S3. $\Delta E_{1 / 2}$ and $i_{p a} / i_{p c}$ values for the cyclic voltammograms of 1-6 at $100 \mathrm{mVs}^{-1}$ in $\mathrm{MeCN}$ with $100 \mathrm{mM}$ of $\left[(n \mathrm{Bu})_{4} \mathrm{~N}\right]\left[\mathrm{PF}_{6}\right]$ as the supporting electrolyte.

Figure S9. Normalized cyclic voltammograms of hypocrellin B (3) in $\mathrm{CH}_{3} \mathrm{CN}$ with 100 $\mathrm{mM}$ of $\left[(n \mathrm{Bu})_{4} \mathrm{~N}\right]\left[\mathrm{PF}_{6}\right]$ as the supporting electrolyte and in $50 \mathrm{mM}$ sodium acetate buffer at $\mathrm{pH}=5$.

Figure S10. Small scale reduction reactions of ent-shiraiachrome A (1), hypocrellin (2), and hypocrellin $\mathrm{B}(\boldsymbol{3})$ with $\mathrm{Na}_{2} \mathrm{~S}_{2} \mathrm{O}_{4}$ under aerobic conditions.

Figure S11. UPLC-HRESIMS chromatogram of ent-shiraiachrome A (1) before and after treatment with $\mathrm{Na}_{2} \mathrm{~S}_{2} \mathrm{O}_{4}$ as a reducing agent overnight.

Figure S12. UPLC-HRESIMS chromatogram of hypocrellin (2) before and after treatment with $\mathrm{Na}_{2} \mathrm{~S}_{2} \mathrm{O}_{4}$ as a reducing agent overnight.

Figure S13. UPLC-HRESIMS chromatogram of hypocrellin B (3) before and after treatment with $\mathrm{Na}_{2} \mathrm{~S}_{2} \mathrm{O}_{4}$ as a reducing agent overnight.

Figure S14. UPLC-HRESIMS chromatograms for the four replicates aerobic reduction reactions of ent-shiraiachrome A (1) with $\mathrm{Na}_{2} \mathrm{~S}_{2} \mathrm{O}_{4}(10 \mathrm{mg}$ of $\mathbf{1}$ was used in each reaction).

Table S4. NMR spectroscopic data for 8-10 in $\mathrm{CDCl}_{3}(\delta$ in ppm). 
Figure S15. ${ }^{1} \mathrm{H}$ - and ${ }^{13} \mathrm{C}\left\{{ }^{1} \mathrm{H}\right\}$-NMR spectra of $8\left[400 \mathrm{MHz}\right.$ for ${ }^{1} \mathrm{H}$ and $100 \mathrm{MHz}$ for $\left.{ }^{13} \mathrm{C}\left\{{ }^{1} \mathrm{H}\right\}, \mathrm{CDCl}_{3}\right]$.

Figure S16. ${ }^{1} \mathrm{H}$ - and ${ }^{13} \mathrm{C}\left\{{ }^{1} \mathrm{H}\right\}$-NMR spectra of $9\left[500 \mathrm{MHz}\right.$ for ${ }^{1} \mathrm{H}$ and $125 \mathrm{MHz}$ for $\left.{ }^{13} \mathrm{C}\left\{{ }^{1} \mathrm{H}\right\}, \mathrm{CDCl}_{3}\right]$.

Figure S17. ${ }^{1} \mathrm{H}$ - and ${ }^{13} \mathrm{C}\left\{{ }^{1} \mathrm{H}\right\}$-NMR spectra of $\mathbf{1 0}\left[700 \mathrm{MHz}\right.$ for ${ }^{1} \mathrm{H}$ and $175 \mathrm{MHz}$ for $\left.{ }^{13} \mathrm{C}\left\{{ }^{1} \mathrm{H}\right\}, \mathrm{CDCl}_{3}\right]$.

Figure S18. HMBC correlations for compounds 8-10.

Figure S19. UV-vis spectra of compounds 1 and 8-10 in methanol at concentration of $0.0125 \mathrm{mg} / \mathrm{mL}$.

Figure S20. ECD spectra for compounds 1-3 and 7 in $\mathrm{CH}_{3} \mathrm{OH}$ at a concentration of 0.2 $\mathrm{mg} / \mathrm{mL}$.

Figure S21. ECD spectra for compounds 8-10 as compared to ent-shiraiachrome A (1) in $\mathrm{CH}_{3} \mathrm{OH}$ at a concentration of $0.2 \mathrm{mg} / \mathrm{mL}$.

Figure S22. UPLC-HRESIMS chromatogram of hypomycin A (4) before and after treatment with $\mathrm{Na}_{2} \mathrm{~S}_{2} \mathrm{O}_{4}$ as a reducing agent overnight.

Figure S23. UPLC-HRESIMS chromatogram of hypomycin C (5) before and after treatment with $\mathrm{Na}_{2} \mathrm{~S}_{2} \mathrm{O}_{4}$ as a reducing agent overnight.

Figure S24. UPLC-HRESIMS chromatogram of hypomycin E (6) before and after treatment with $\mathrm{Na}_{2} \mathrm{~S}_{2} \mathrm{O}_{4}$ as a reducing agent overnight.

Figure S25. Small scale reduction reactions of hypomycin A (4), C (5), and E (6) with $\mathrm{Na}_{2} \mathrm{~S}_{2} \mathrm{O}_{4}$ under aerobic conditions.

Figure S26. UV-vis spectra of compounds 1-6 in methanol at concentration of 0.0125 $\mathrm{mg} / \mathrm{mL}$.

Figure S27. UPLC-HRESIMS chromatograms for the triplicate anaerobic reduction reactions of ent-shiraiachrome A (1) with $\mathrm{Na}_{2} \mathrm{~S}_{2} \mathrm{O}_{4}$.

Figure S28. UPLC-HRESIMS chromatograms for the anaerobic reduction reactions of ent-shiraiachrome A (1) as compared to UPLC-HRESIMS chromatograms of pure standards of 2-6.

Figure S29. ECD spectra in $\mathrm{CH}_{3} \mathrm{OH}$ for compounds 4-6 and $\mathbf{1 1}$ which were isolated from the anaerobic reduction reaction of $\mathbf{1}$ and $\mathbf{2}$.

Figure S30. Proposed generation of hypomycin A (4) via reduction of hypocrellin (2) with $\mathrm{Na}_{2} \mathrm{~S}_{2} \mathrm{O}_{4}$ under anaerobic conditions. 
Figure S31. ${ }^{1} \mathrm{H}-\mathrm{NMR}$ of ent-shiraiachrome A (1) in DMSO- $d_{6}(\mathbf{A})$ and ${ }^{1} \mathrm{H}-\mathrm{NMR}$ of $\mathbf{1}$ in $\mathrm{CDCl}_{3}$ after exposure to DMSO- $d_{6}(\mathbf{B})$.

Figure S32. ${ }^{1} \mathrm{H}-\mathrm{NMR}$ of pure ent-shiraiachrome $\mathrm{A}(\mathbf{1})$ in $\mathrm{CDCl}_{3}$ before and after exposure to DMSO vs ${ }^{1} \mathrm{H}-\mathrm{NMR}$ of hypocrellin (2) in $\mathrm{CDCl}_{3}$.

Figure S33. Typical single crystals of hypocrellin B (3) suitable for X-ray diffraction analysis, prepared via $\mathrm{ENaCt}$ after 14 days.

Figure S34. Displacement ellipsoid plot (50\% probability level) of hypocrellin B (3), showing the atom-labelling scheme. Hydrogen atoms and solvent molecule have been omitted for clarity.

Table S5. Crystal data and structure refinement for hypocrellin B (3).

Figure S35. ${ }^{1} \mathrm{H}-\mathrm{NMR}$ for the anaerobic reduction reaction mixture of ent-shiraiachrome A (1) as compared to hypomycin E (6) in acetonitrile- $d_{3}$.

Figure S36. ${ }^{1} \mathrm{H}-\mathrm{NMR}$ for the anaerobic reduction reaction mixture of hypocrellin (2) as compared to hypomycin A (4) in acetonitrile- $d_{3}$.

Figure S37. Previously reported structures of hypomycin C (5) and E (6) as compared to ent-shiraiachrome A (1). ${ }^{6}$

Figure S38. Typical microcrystals of ent-shiraiachrome A (1), prepared via ENaCt.

Figure S39. Single crystals of ent-shiraiachrome A (1) suitable for X-ray diffraction analysis, prepared via $\mathrm{ENaCt}$ after 14 days.

Figure S40. Displacement ellipsoid plot (50\% probability level) of ent-shiraiachrome A (1), showing the atom-labelling scheme. Hydrogen atoms have been omitted for clarity.

Table S6. Crystal data and structure refinement for ent-shiraiachrome A (1).

Figure S41. Key NOESY correlations of hypomycin C (5) and hypomycin E (6).

Figure S42. The observed intramolecular hydrogen-bonding of the exchangeable protons in hypomycin A (4), C (5), and E (6).

Figure S43. ${ }^{1} \mathrm{H}-\mathrm{NMR}$ of hypomycin A (4) [400 MHz, $\left.\mathrm{CDCl}_{3}\right]$.

Figure S44. ${ }^{1} \mathrm{H}-\mathrm{NMR}$ of hypomycin C (5) $\left[400 \mathrm{MHz}, \mathrm{CDCl}_{3}\right]$.

Figure S45. ${ }^{1} \mathrm{H}-\mathrm{NMR}$ of hypomycin C (5) [400 MHz, DMSO- $\left.d_{6}\right] . \quad$ S- 56

Figure S46. ${ }^{1} \mathrm{H}-\mathrm{NMR}$ of hypomycin $\mathrm{E}(\mathbf{6})$ [400 $\left.\mathrm{MHz}, \mathrm{CDCl}_{3}\right]$. 
Figure S47. Experimental ECD spectrum of $\mathbf{5}$ compared with calculated ECD spectra of the newly-proposed configuration of $5[P(S), 1 R, 2 S, 14 R, 15 R, 17 S]$ on the left frame and the previously proposed configuration of $5[P(S), 1 S, 2 R, 14 S, 15 S, 17 R]$ on the right frame.

Table S7. NMR spectroscopic data for 11-12 in $\mathrm{CDCl}_{3}(\delta$ in ppm).

Figure S48. ${ }^{1} \mathrm{H}$ - and ${ }^{13} \mathrm{C}\left\{{ }^{1} \mathrm{H}\right\}-\mathrm{NMR}$ spectra of $11\left[400 \mathrm{MHz}\right.$ for ${ }^{1} \mathrm{H}$ and $100 \mathrm{MHz}$ for $\left.{ }^{13} \mathrm{C}\left\{{ }^{1} \mathrm{H}\right\}, \mathrm{CDCl}_{3}\right]$.

Figure S49. ${ }^{1} \mathrm{H}$ - and ${ }^{13} \mathrm{C}\left\{{ }^{1} \mathrm{H}\right\}$-NMR spectra of $12\left[700 \mathrm{MHz}\right.$ for ${ }^{1} \mathrm{H}$ and $175 \mathrm{MHz}$ for $\left.{ }^{13} \mathrm{C}\left\{{ }^{1} \mathrm{H}\right\}, \mathrm{CDCl}_{3}\right]$.

Figure S50. HMBC correlations for compounds 11 and 12. 


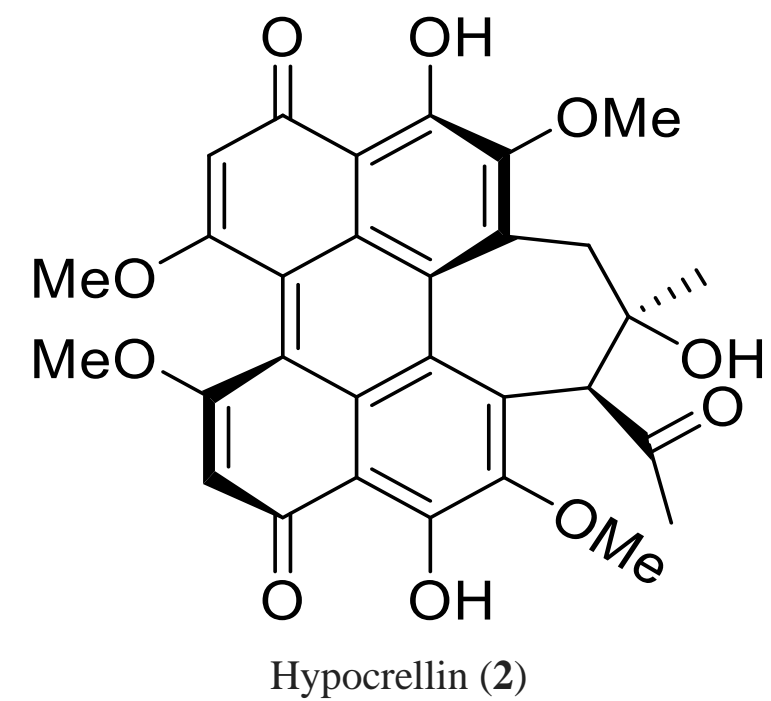

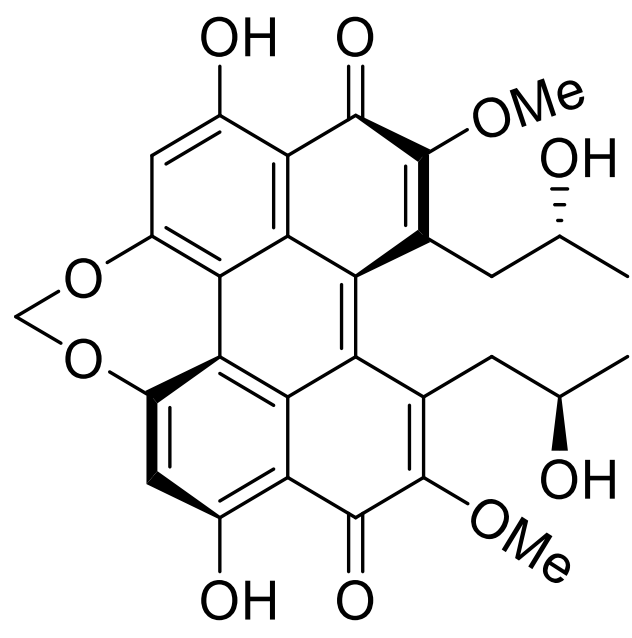

Cercosporin

Figure S1. Structural similarity between hypocrellin (2) and cercosporin. 

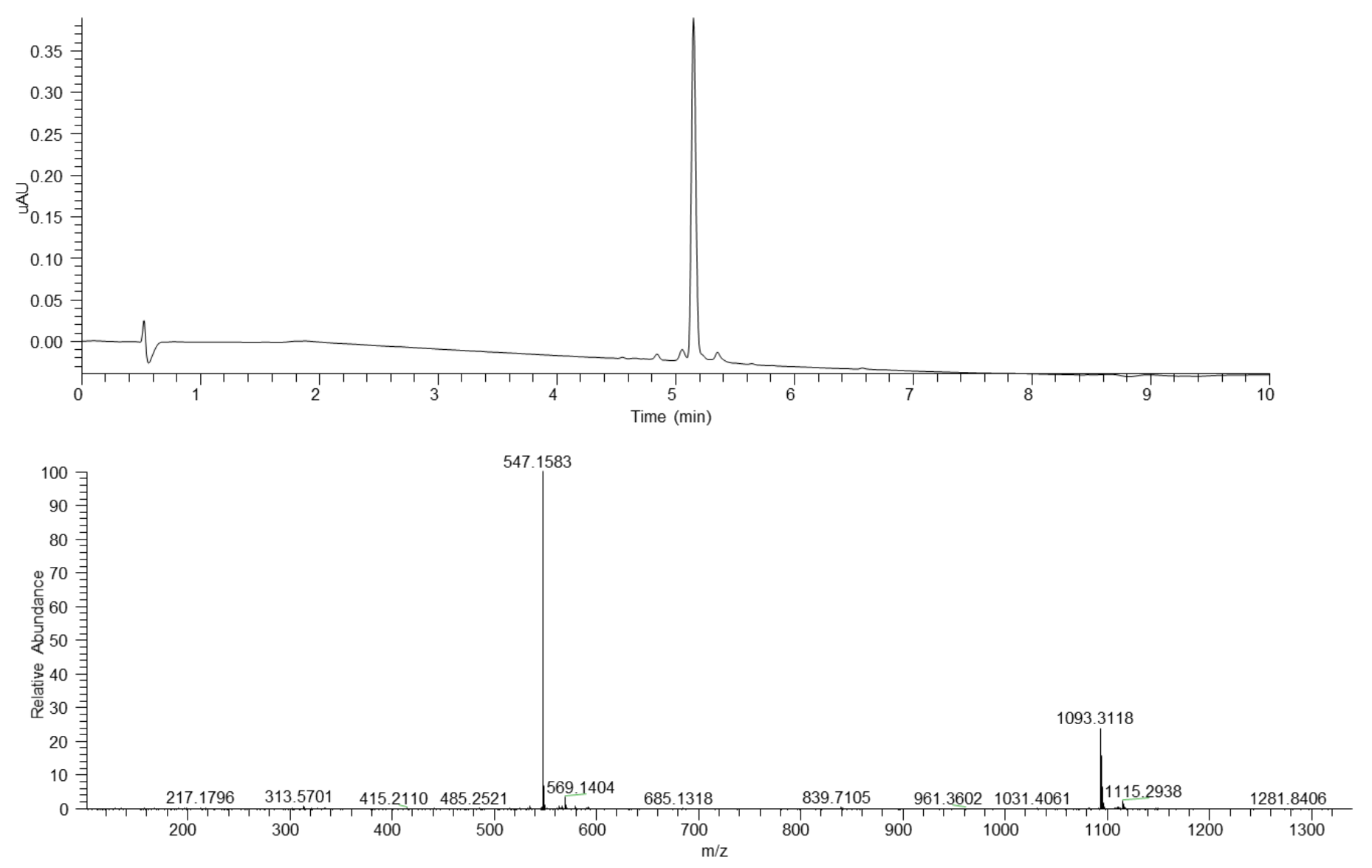

Figure S2. UPLC-(+)-HRESIMS chromatogram of 7. 


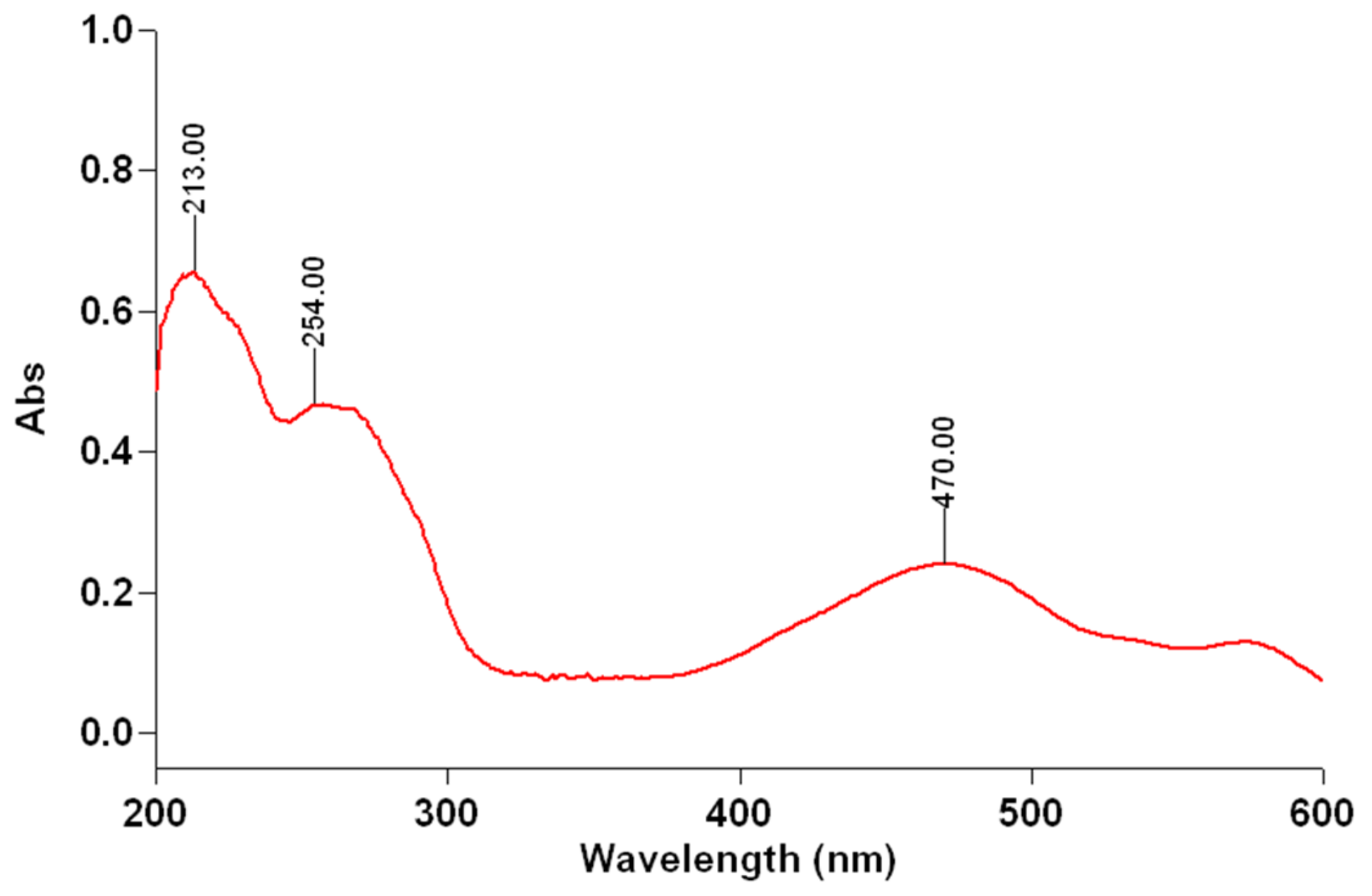

Figure S3. UV-vis spectrum of 7 in methanol at concentration of $0.0125 \mathrm{mg} / \mathrm{mL}$. 
Table S1. NMR Spectroscopic Data for $\mathbf{7}$ in $\mathrm{CDCl}_{3}\left(700 \mathrm{MHz}\right.$ for ${ }^{1} \mathrm{H}$ and $175 \mathrm{MHz}$ for ${ }^{13} \mathrm{C}\left\{{ }^{1} \mathrm{H}\right\}, \delta$ in ppm)

\begin{tabular}{lll} 
& \multicolumn{2}{c}{7} \\
\cline { 2 - 3 } Position & $\delta_{\mathrm{C}, \text { type }}$ & $\delta_{\mathrm{H}}(\mathrm{J}$ in Hz $)$ \\
$1 \mathrm{a}, 12 \mathrm{a}$ & $132.5, \mathrm{C}$ & \\
2,11 & $125.6, \mathrm{C}$ & \\
3,10 & $171.3, \mathrm{C}$ & \\
$3 \mathrm{a}, 9 \mathrm{a}$ & $107.1, \mathrm{C}$ & \\
$3 \mathrm{~b}, 9 \mathrm{~b}$ & $126.3, \mathrm{C}$ & \\
4,9 & $179.7, \mathrm{C}$ & \\
5,8 & $102.5, \mathrm{CH}$ & $6.53, \mathrm{~s}$ \\
6,7 & $165.8, \mathrm{C}$ & \\
$6 \mathrm{a}, 7 \mathrm{a}$ & $119.2, \mathrm{C}$ & \\
13,16 & $47.7, \mathrm{CH}_{2}$ & $3.61, \mathrm{~d}(17.4)$ \\
& & $4.06, \mathrm{~d}(17.4)$ \\
14,17 & $204.6, \mathrm{C}$ & \\
15,18 & $30.0, \mathrm{CH}_{3}$ & $2.26, \mathrm{~s}$ \\
$\mathrm{CH}_{3} \mathrm{O}-2$, & $60.6, \mathrm{CH}_{3}$ & $4.12, \mathrm{~s}$ \\
$\mathrm{CH}_{3} \mathrm{O}-11$ & & \\
$\mathrm{CH}_{3} \mathrm{O}-6$, & $56.7, \mathrm{CH}_{3}$ & $3.92, \mathrm{~s}$ \\
$\mathrm{CH}_{3} \mathrm{O}-7$ & & \\
$\mathrm{HO}_{3} 3$ & & $15.93, \mathrm{~s}$ \\
$\mathrm{HO}_{-10}$ & & \\
\hline
\end{tabular}




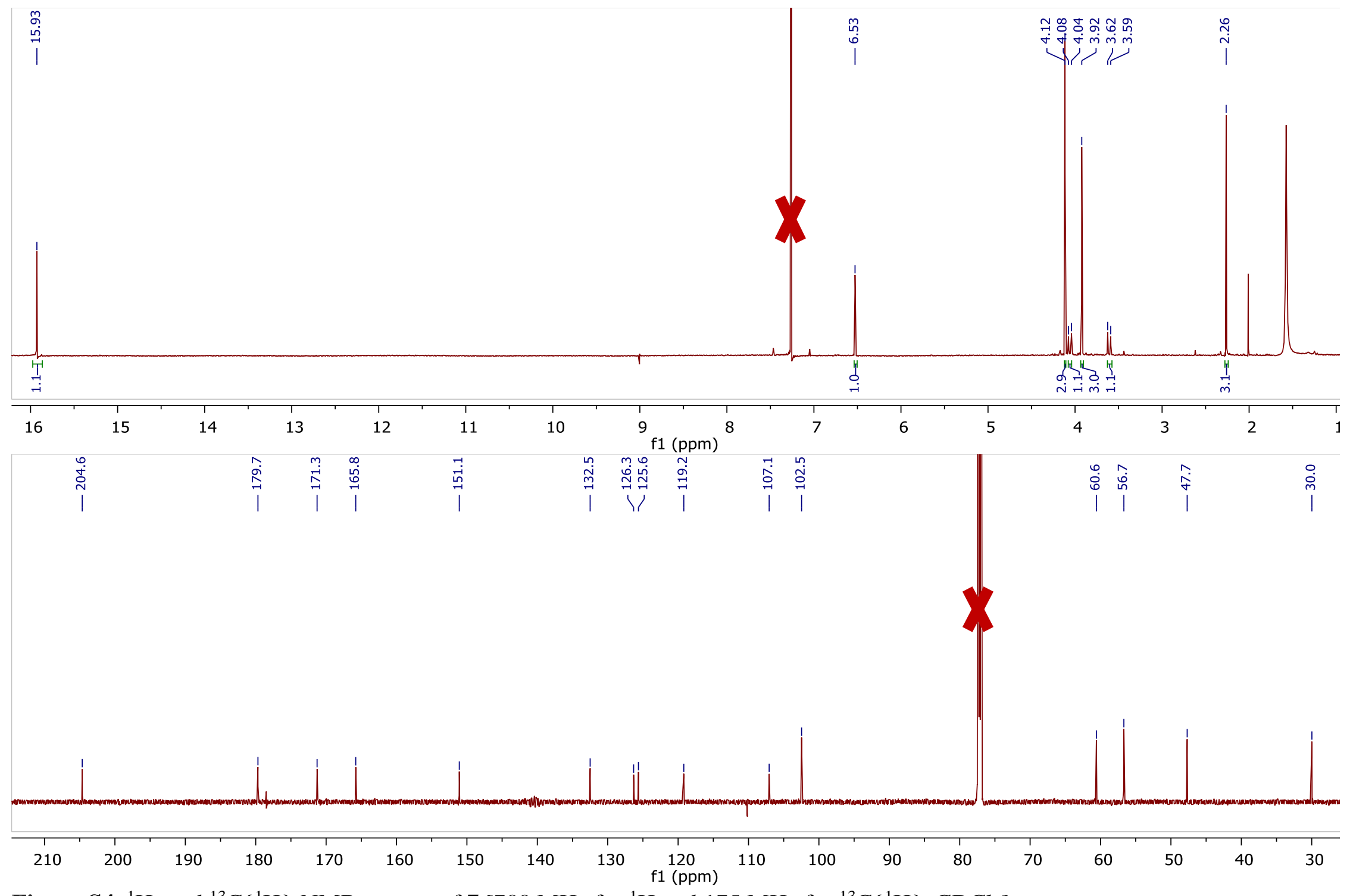

Figure S4. ${ }^{1} \mathrm{H}$ - and ${ }^{13} \mathrm{C}\left\{{ }^{1} \mathrm{H}\right\}$-NMR spectra of $7\left[700 \mathrm{MHz}\right.$ for ${ }^{1} \mathrm{H}$ and $175 \mathrm{MHz}$ for $\left.{ }^{13} \mathrm{C}\left\{{ }^{1} \mathrm{H}\right\}, \mathrm{CDCl}_{3}\right]$. 


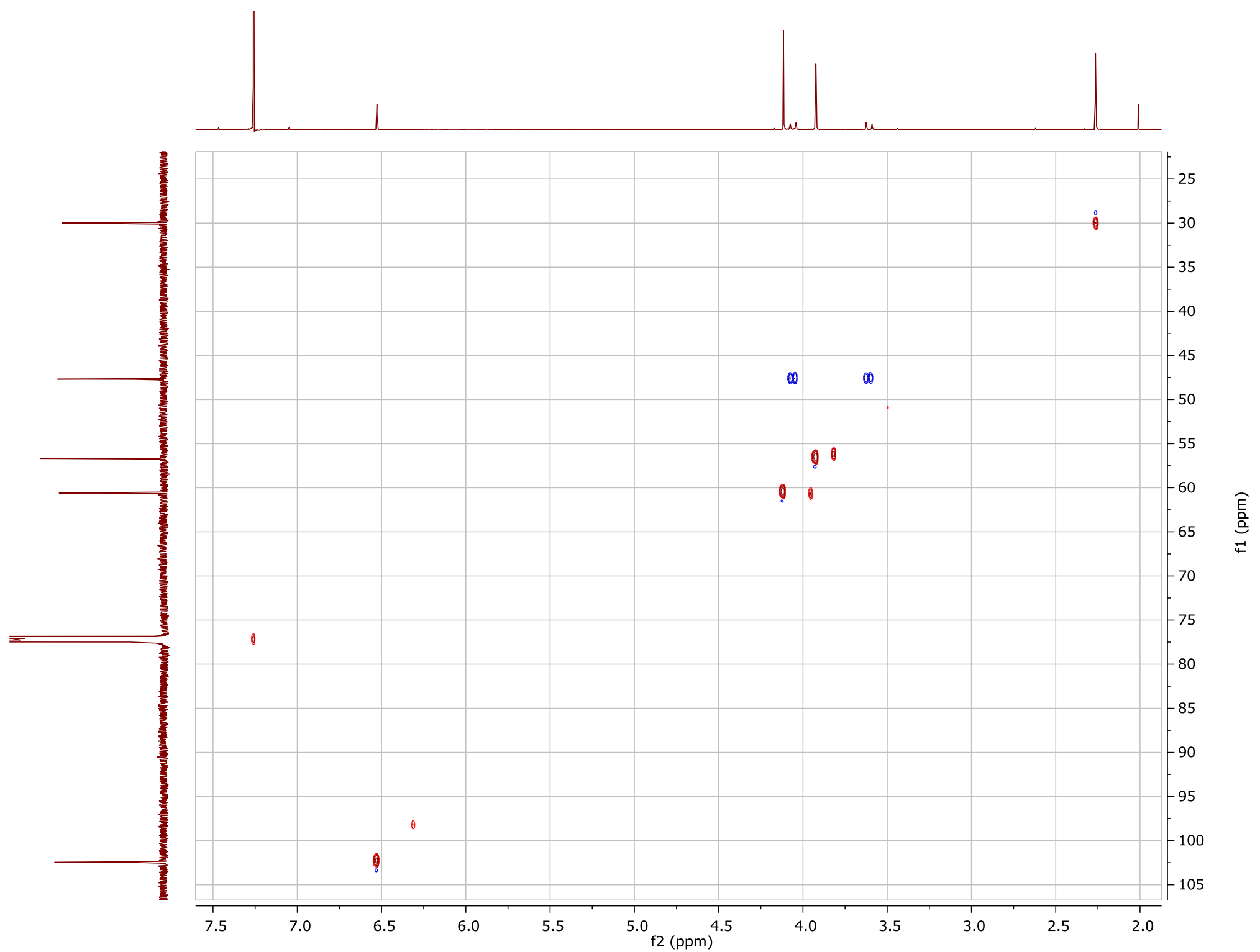

Figure S5. Edited-HSQC NMR spectrum of 7 [700 MHz, $\left.\mathrm{CDCl}_{3}\right]$. 


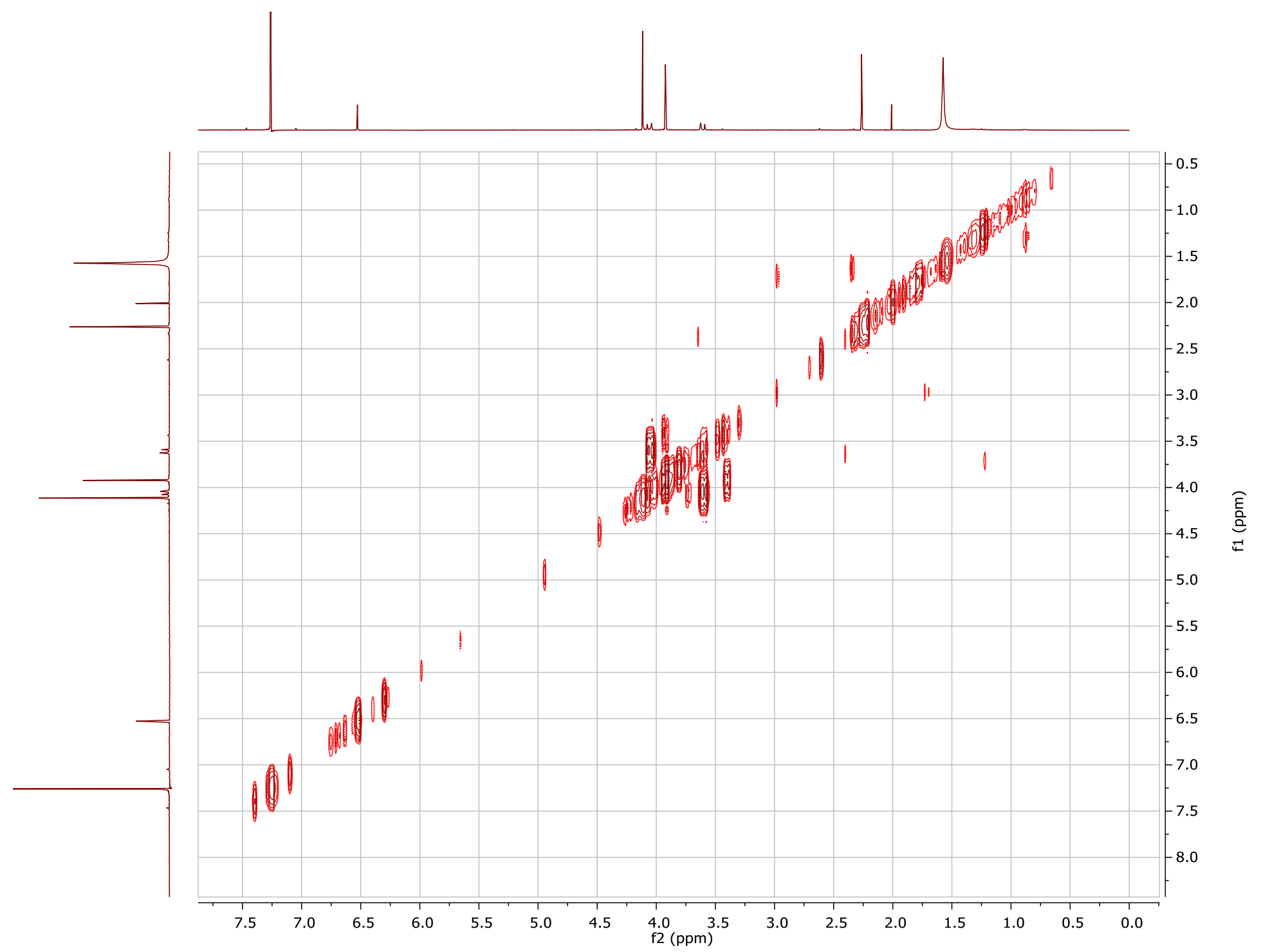

Figure S6. COSY NMR spectrum of 7 [700 MHz, $\left.\mathrm{CDCl}_{3}\right]$. 


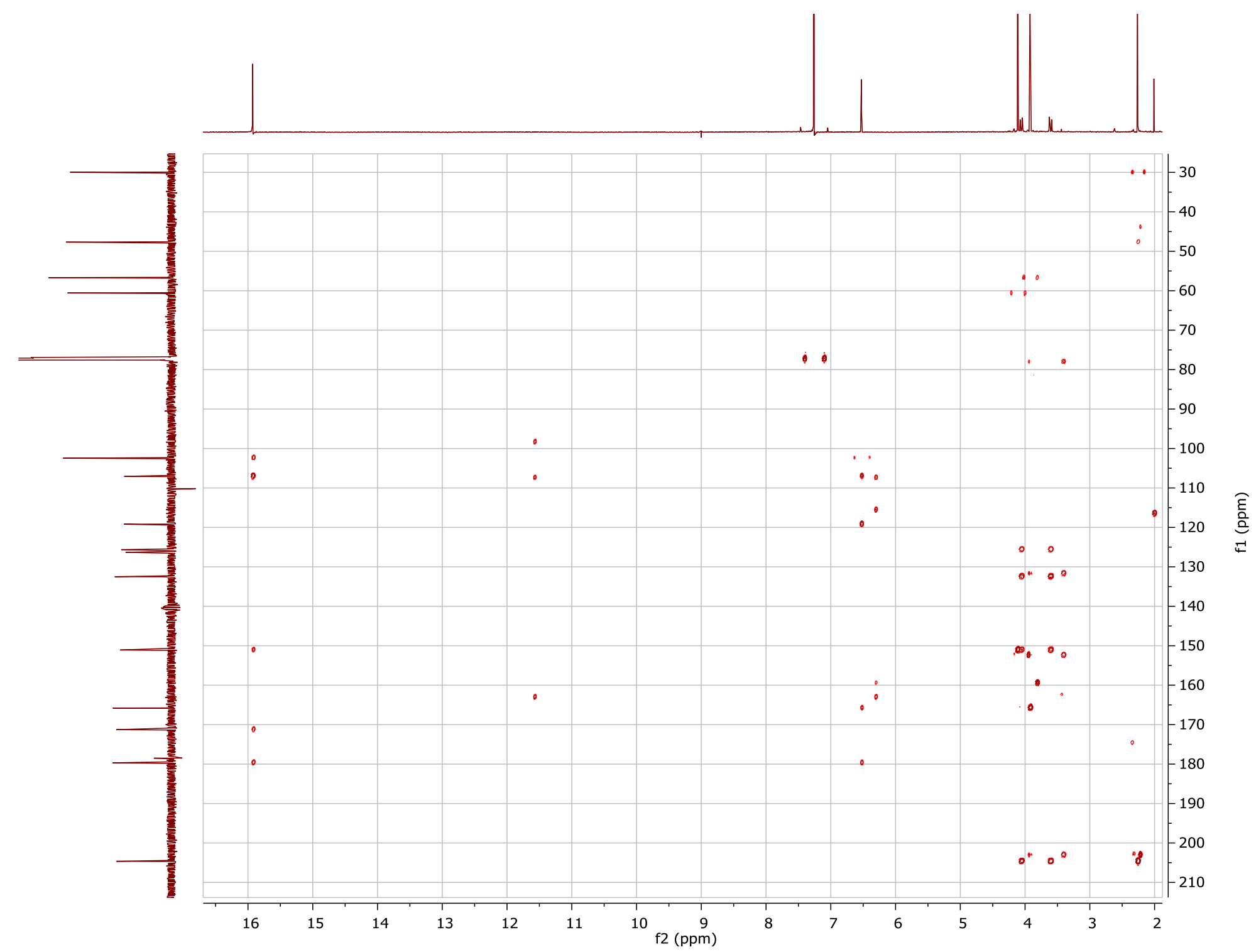

Figure S7. $\mathrm{HMBC}$ NMR spectrum of 7 [700 MHz, $\left.\mathrm{CDCl}_{3}\right]$. 


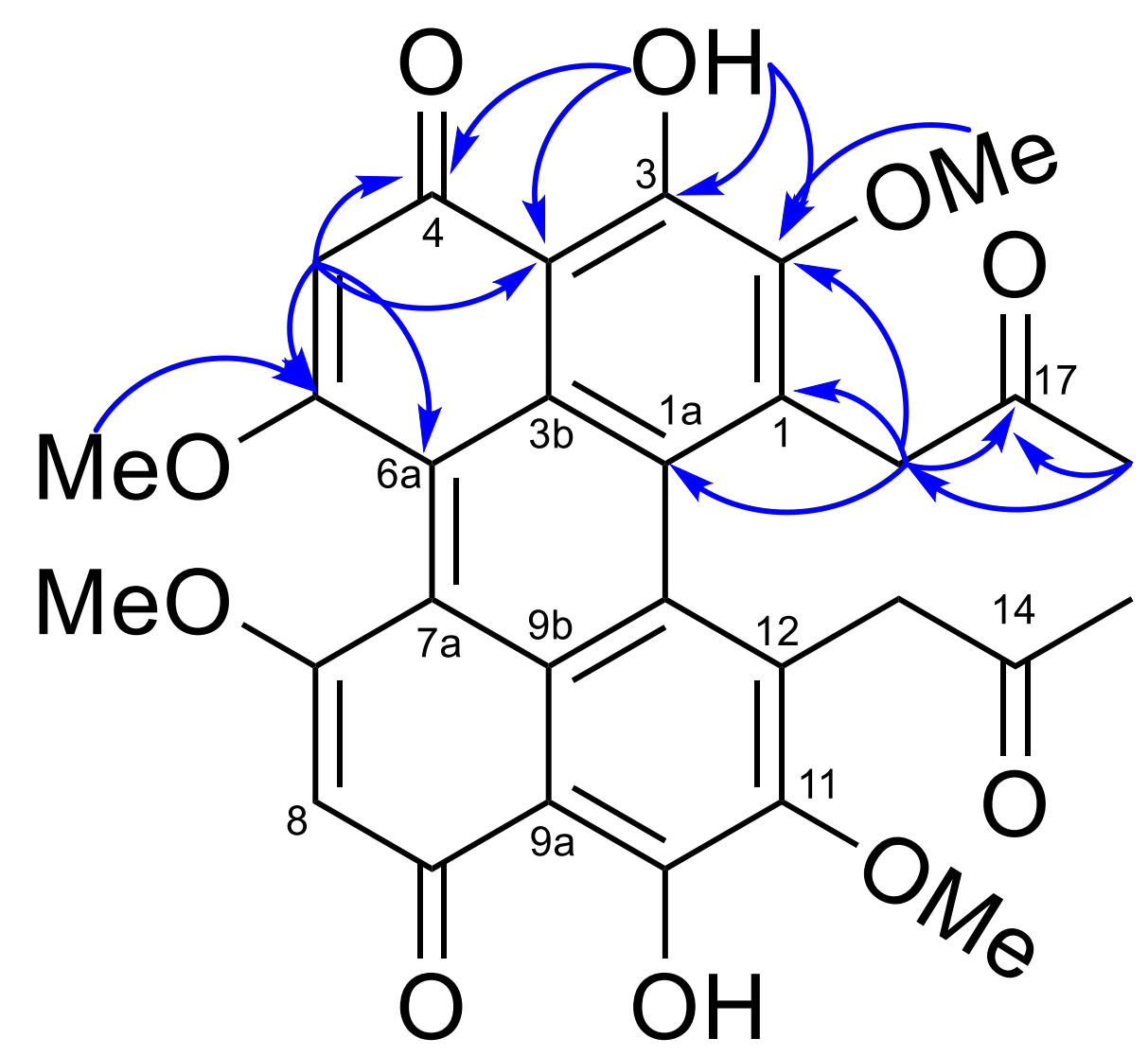

Figure S8. Key HMBC correlations of 7. 
Table S2. $E_{1 / 2}$ values for the cyclic voltammograms of 1-6 in $\mathrm{MeCN}$ with $100 \mathrm{mM}$ of $\left[(n \mathrm{Bu})_{4} \mathrm{~N}\right]\left[\mathrm{PF}_{6}\right]$ as the supporting electrolyte.

\begin{tabular}{ccc}
\hline & \multicolumn{2}{c}{$E_{1 / 2}(\mathrm{~V}$ vs Ag/AgCl $)$} \\
& $1^{\text {st }}$ Reduction & $2^{\text {nd }}$ Reduction \\
\hline ent-Shiraiachrome A (1) & -0.514 & -0.756 \\
Hypocrellin (2) & -0.503 & -0.756 \\
Hypocrellin B (3) & -0.464 & -0.709 \\
Hypomycin A (4) & -0.960 & -1.302 \\
Hypomycin C (5) & -1.001 & -1.290 \\
Hypomycin E (6) & -1.050 & -1.364 \\
\hline
\end{tabular}


Table S3. $\Delta E_{1 / 2}$ and $i_{p a} / i_{p c}$ values for the cyclic voltammograms of 1-6 at $100 \mathrm{mVs}^{-1}$ in $\mathrm{MeCN}$ with $100 \mathrm{mM}$ of $\left[(n \mathrm{Bu})_{4} \mathrm{~N}\right]\left[\mathrm{PF}_{6}\right]$ as the supporting electrolyte.

\begin{tabular}{ccccc}
\hline & \multicolumn{2}{c}{$\Delta E_{1 / 2}(\mathrm{~V}$ vs $\mathrm{Ag} / \mathrm{AgCl})$} & at $100 \mathrm{mVs}^{-1}$ & \multicolumn{2}{c}{$i_{p a} / i_{p c}$ at $100 \mathrm{mVs}^{-1}$} \\
& $1^{\text {st }}$ Reduction & $2^{\text {nd }}$ Reduction & $1^{\text {st }}$ Reduction & $2^{\text {nd }}$ Reduction \\
\hline ent-Shiraiachrome A (1) & 0.064 & 0.068 & 0.90 & 1.05 \\
Hypocrellin (2) & 0.071 & 0.079 & 0.93 & 1.09 \\
Hypocrellin B (3) & 0.065 & 0.070 & 0.93 & 1.03 \\
Hypomycin A (4) & 0.064 & 0.080 & 1.10 & 1.04 \\
Hypomycin C (5) & 0.060 & 0.060 & 1.12 & 1.04 \\
Hypomycin E (6) & 0.060 & 0.070 & 1.06 & 1.02 \\
\hline
\end{tabular}




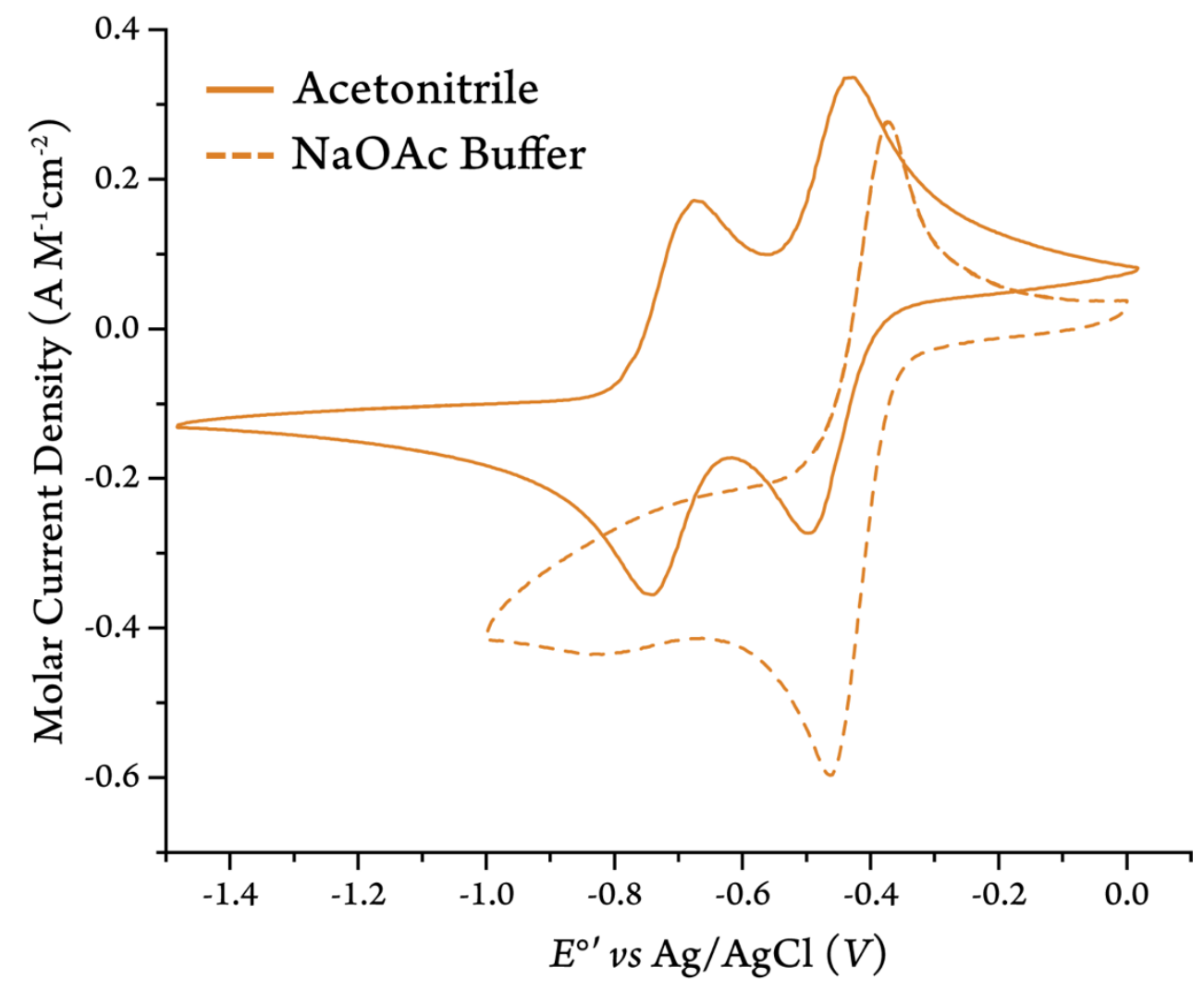

Figure S9. Normalized cyclic voltammograms of hypocrellin B (3) in $\mathrm{CH}_{3} \mathrm{CN}$ with $100 \mathrm{mM}$ of $\left[(n \mathrm{Bu})_{4} \mathrm{~N}\right]\left[\mathrm{PF}_{6}\right]$ as the supporting electrolyte and in $50 \mathrm{mM}$ sodium acetate buffer at $\mathrm{pH}=5$. 


\section{A: Deep red colored solutions}

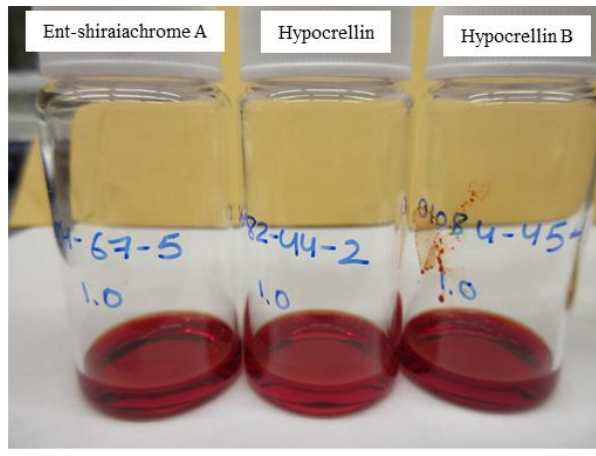

$1 \mathrm{mg} / \mathrm{mL}$ of each compound dissolved acetonitrile
B: Yellow colored solutions


C: Superficial red-colored layers

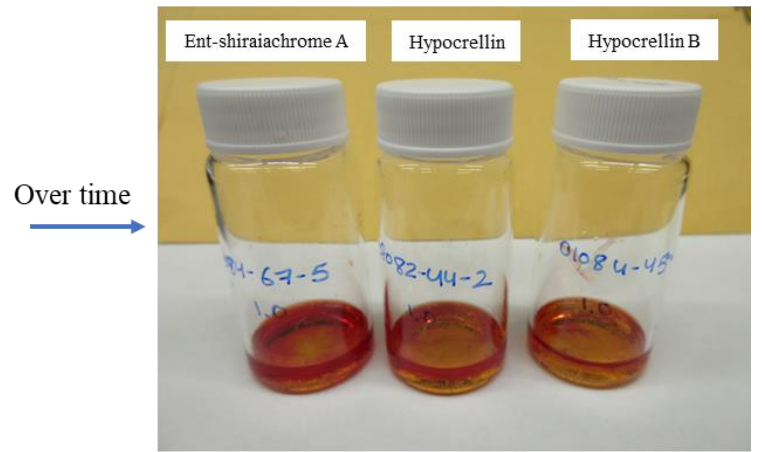

Figure S10. Small scale reduction reactions of ent-shiraiachrome A (1), hypocrellin (2), and hypocrellin B (3) with $\mathrm{Na}_{2} \mathrm{~S}_{2} \mathrm{O}_{4}$ under aerobic conditions. 


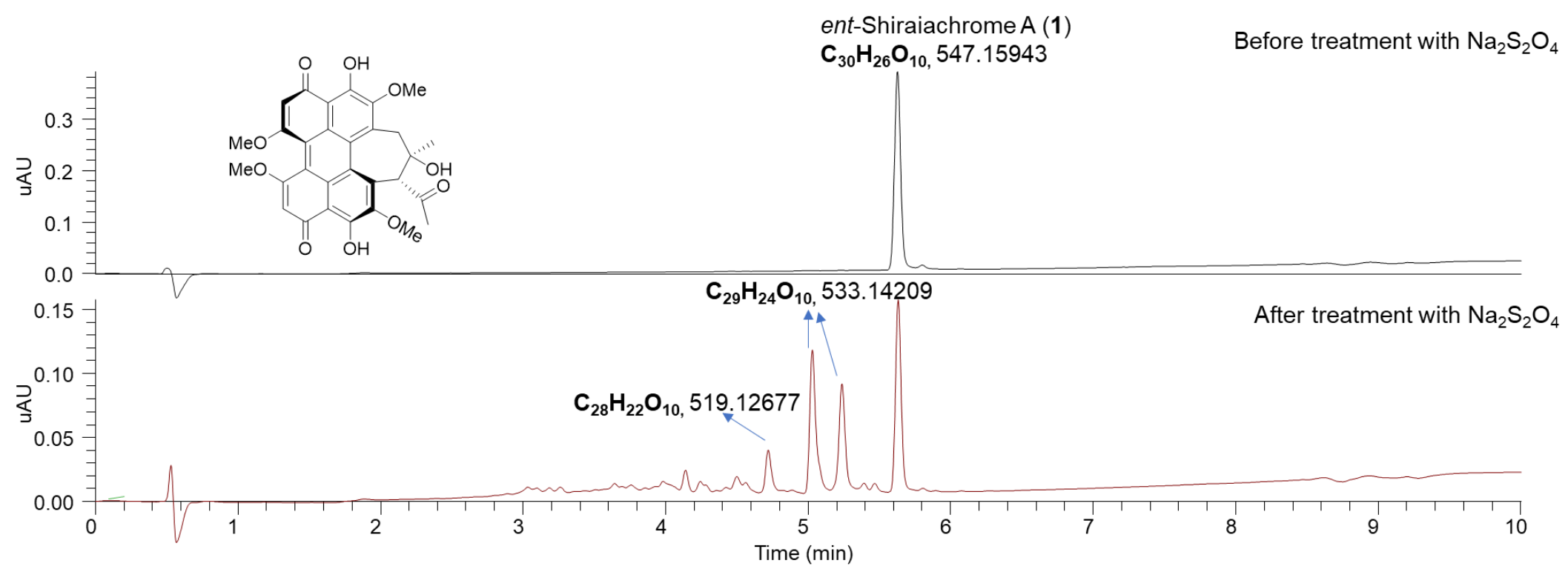

Figure S11. UPLC-HRESIMS chromatogram of ent-shiraiachrome A (1) before and after treatment with $\mathrm{Na}_{2} \mathrm{~S}_{2} \mathrm{O}_{4}$ as a reducing agent overnight. 


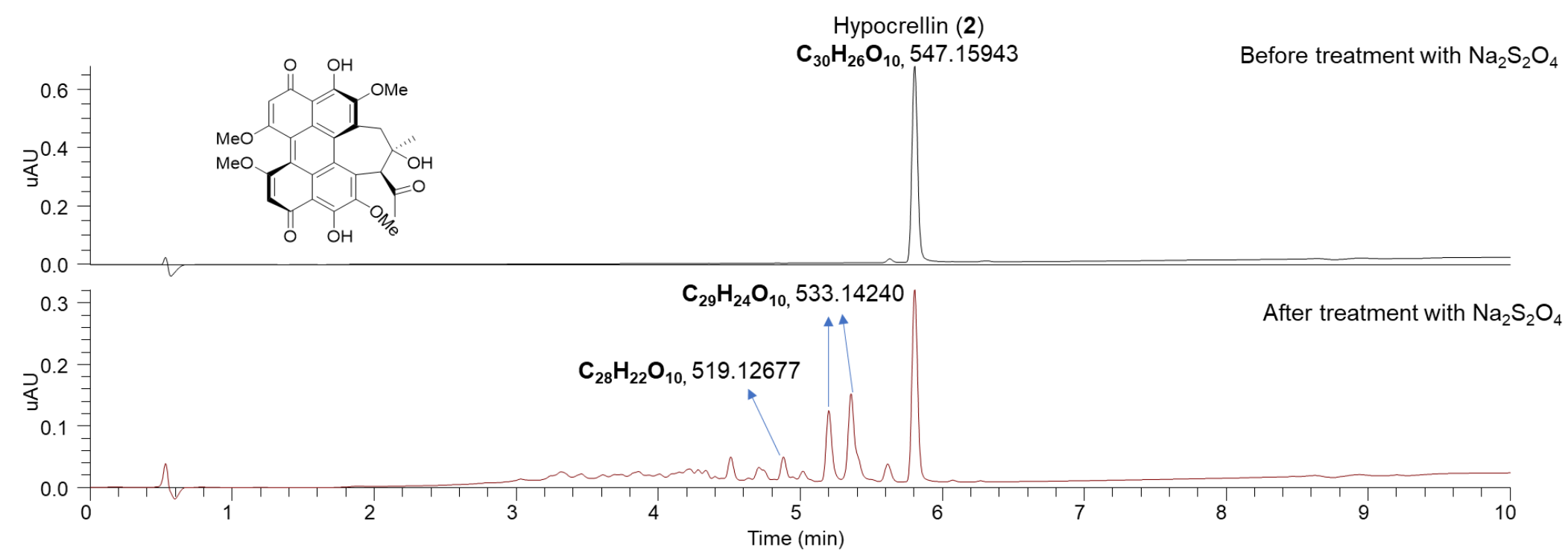

Figure S12. UPLC-HRESIMS chromatogram of hypocrellin (2) before and after treatment with $\mathrm{Na}_{2} \mathrm{~S}_{2} \mathrm{O}_{4}$ as a reducing agent overnight. 


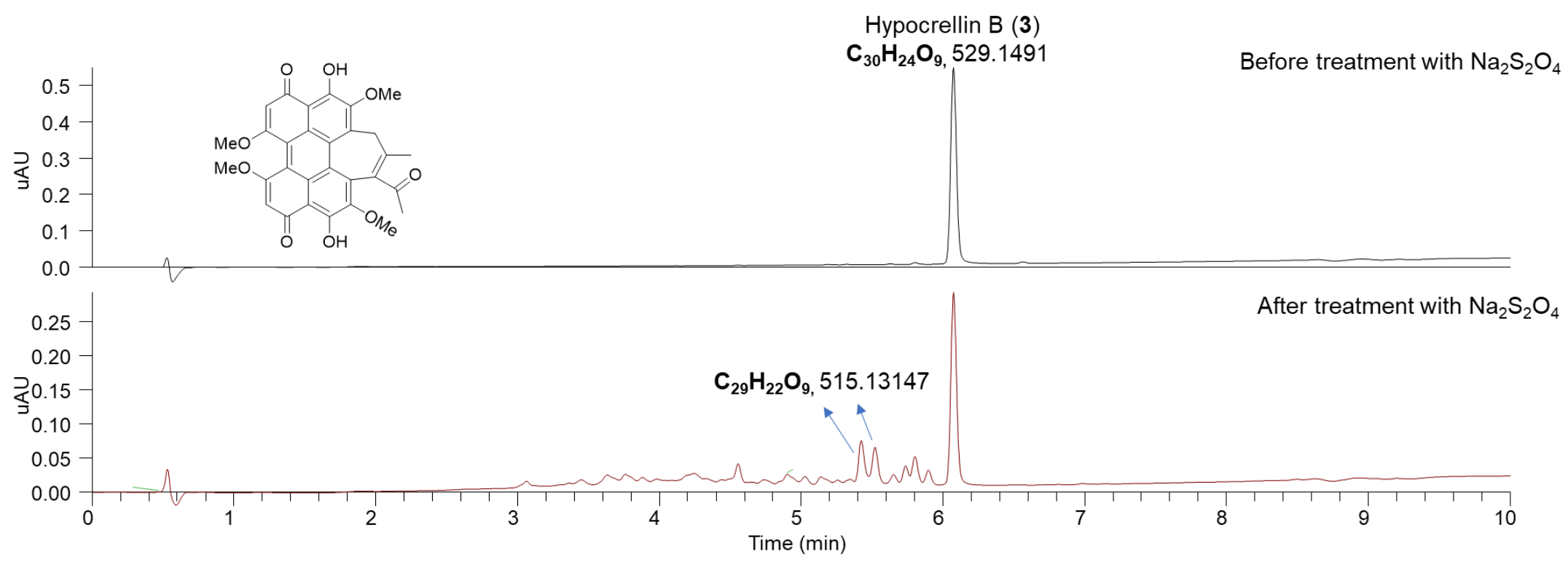

Figure S13. UPLC-HRESIMS chromatogram of hypocrellin B (3) before and after treatment with $\mathrm{Na}_{2} \mathrm{~S}_{2} \mathrm{O}_{4}$ as a reducing agent overnight. 


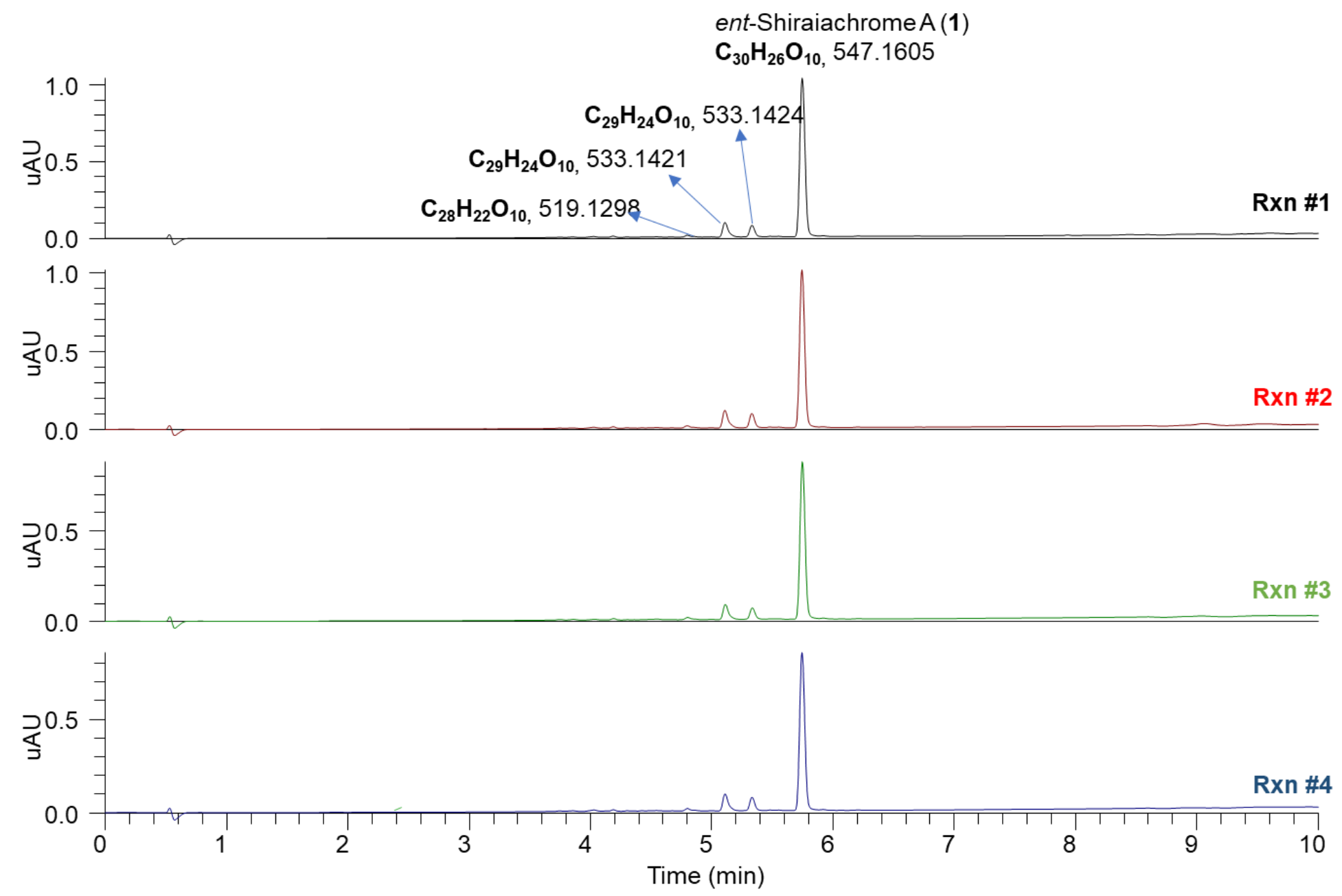

Figure S14. UPLC-HRESIMS chromatograms for the four replicates aerobic reduction reactions of ent-shiraiachrome A (1) with $\mathrm{Na}_{2} \mathrm{~S}_{2} \mathrm{O}_{4}$ (10 mg of 1 was used in each reaction). 
Table S4. NMR Spectroscopic Data for 8-10 in $\mathrm{CDCl}_{3}(\delta$ in ppm).

\begin{tabular}{|c|c|c|c|c|c|c|}
\hline \multirow[b]{2}{*}{ position } & \multicolumn{2}{|r|}{$8^{\mathrm{a}}$} & \multicolumn{2}{|r|}{$9^{b}$} & \multicolumn{2}{|r|}{$10^{c}$} \\
\hline & $\delta_{\mathrm{C}}$, type & $\delta_{\mathrm{H}}(J, \mathrm{~Hz})$ & $\delta_{\mathrm{C}}$, type & $\delta_{\mathrm{H}}(J, \mathrm{~Hz})$ & $\delta_{\mathrm{C}}$, type & $\delta_{\mathrm{H}}(J, \mathrm{~Hz})$ \\
\hline 1 & $135.0, \mathrm{C}$ & & $121.9, \mathrm{C}$ & & $121.9, \mathrm{C}$ & \\
\hline $1 \mathrm{a}$ & 129.7, C & & $130.8, \mathrm{C}$ & & 130.9, C & \\
\hline 2 & $153.1, \mathrm{C}$ & & $149.4, \mathrm{C}$ & & $149.5, \mathrm{C}$ & \\
\hline 3 & $178.5, \mathrm{C}$ & & $176.5, \mathrm{C}$ & & 177.6, C & \\
\hline $3 a$ & 106.1, C & & 103.9, C & & 103.9, C & \\
\hline $3 b$ & 125.5, C or & & 125.6, $\mathrm{C}$ or & & $125.8, \mathrm{C}$ or & \\
\hline 4 & $\begin{array}{l}126.6, \mathrm{C} \\
174.0, \mathrm{C}\end{array}$ & & $\begin{array}{l}125.9, \mathrm{C} \\
171.7, \mathrm{C}\end{array}$ & & $\begin{array}{l}126.2, \mathrm{C} \\
170.5, \mathrm{C}\end{array}$ & \\
\hline 5 & $100.7, \mathrm{CH}$ & $6.71, \mathrm{~s}$ & $99.8, \mathrm{CH}$ & $6.74, \mathrm{~s}$ & $99.5, \mathrm{CH}$ & $6.77, \mathrm{~s}$ \\
\hline 6 & 166.4, C & & 167.5, C & & 167.1, C & \\
\hline $6 a$ & $114.5, \mathrm{C}$ & & $115.0, \mathrm{C}$ & & $\begin{array}{l}113.8, \mathrm{C} \text { or } \\
114.1, \mathrm{C}\end{array}$ & \\
\hline 7 & 168.0, C & & 166.7, C & & 167.6, C & \\
\hline $7 \mathrm{a}$ & 115.1, C & & $113.9, \mathrm{C}$ & & $\begin{array}{l}113.8, \mathrm{C} \text { or } \\
114.1, \mathrm{C}\end{array}$ & \\
\hline 8 & $99.6, \mathrm{CH}$ & $6.75, \mathrm{~s}$ & $100.2, \mathrm{CH}$ & $6.72, \mathrm{~s}$ & $99.3, \mathrm{CH}$ & $6.77, \mathrm{~s}$ \\
\hline 9 & $171.8, \mathrm{C}$ & & 173.3, C & & $170.5, \mathrm{C}$ & \\
\hline $9 a$ & 104.0, C & & 106.4, C & & 104.0, C & \\
\hline $9 b$ & $\begin{array}{l}125.5, \mathrm{C} \text { or } \\
126.6, \mathrm{C}\end{array}$ & & $\begin{array}{l}125.6, \mathrm{C} \text { or } \\
125.9, \mathrm{C}\end{array}$ & & $\begin{array}{l}125.8, \mathrm{C} \text { or } \\
126.2, \mathrm{C}\end{array}$ & \\
\hline 10 & 176.0, C & & 178.8, C & & 177.1, C & \\
\hline 11 & $147.4, \mathrm{C}$ & & $150.2, \mathrm{C}$ & & $147.4, \mathrm{C}$ & \\
\hline 12 & 120.7, C & & $129.4, \mathrm{C}$ & & 120.7, C & \\
\hline $12 \mathrm{a}$ & $130.4, \mathrm{C}$ & & 131.3, C & & 130.0, C & \\
\hline 13 & $42.8, \mathrm{CH}_{2}$ & $2.40, \mathrm{~d}(13.6)$ & $42.5, \mathrm{CH}_{2}$ & $2.42, \mathrm{~d}(14.0)$ & $42.6, \mathrm{CH}_{2}$ & $2.47, \mathrm{~d}(14.2)$ \\
\hline & & $3.64, \mathrm{~d}(13.6)$ & & $3.54, \mathrm{~d}(14.0)$ & & $3.54, \mathrm{~d}(14.2)$ \\
\hline 14 & 78.0, C & & 78.3, C & & 78.7, C & \\
\hline 15 & $64.4, \mathrm{CH}$ & $3.76, \mathrm{~s}$ & $64.6, \mathrm{CH}$ & $3.78, \mathrm{~s}$ & $64.6, \mathrm{CH}$ & $3.84, \mathrm{~s}$ \\
\hline
\end{tabular}




\begin{tabular}{|c|c|c|c|c|c|c|}
\hline 16 & $25.0, \mathrm{CH}_{3}$ & $1.77, \mathrm{~s}$ & $24.8, \mathrm{CH}_{3}$ & $1.78, \mathrm{~s}$ & $25.0, \mathrm{CH}_{3}$ & $1.79, \mathrm{~s}$ \\
\hline 17 & $207.8, \mathrm{C}$ & & 206.7, C & & 207.8, C & \\
\hline 18 & $28.7, \mathrm{CH}_{3}$ & $1.92, \mathrm{~s}$ & $28.4, \mathrm{CH}_{3}$ & $1.85, \mathrm{~s}$ & 28.6, $\mathrm{CH}_{3}$ & $1.91, \mathrm{~s}$ \\
\hline $\mathrm{CH}_{3} \mathrm{O}-2$ & $61.9, \mathrm{CH}_{3}$ & $4.18, \mathrm{~s}$ & & & & \\
\hline $\mathrm{CH}_{3} \mathrm{O}-6$ & $56.6, \mathrm{CH}_{3}$ & $4.10, \mathrm{~s}$ & $56.7, \mathrm{CH}_{3}$ & $4.12, \mathrm{~s}$ & $\begin{array}{l}56.7, \mathrm{CH}_{3} \text { or } \\
56.8, \mathrm{CH}_{3}\end{array}$ & $4.13, \mathrm{~s}$ \\
\hline $\mathrm{CH}_{3} \mathrm{O}-7$ & $56.9, \mathrm{CH}_{3}$ & $4.13, \mathrm{~s}$ & $56.7, \mathrm{CH}_{3}$ & $4.10, \mathrm{~s}$ & $\begin{array}{l}56.7, \mathrm{CH}_{3} \text { or } \\
56.8, \mathrm{CH}_{3}\end{array}$ & $4.13, \mathrm{~s}$ \\
\hline $\mathrm{CH}_{3} \mathrm{O}-11$ & & & 61.0, $\mathrm{CH}_{3}$ & $4.27, \mathrm{~s}$ & & \\
\hline HO-2 & & & & & & $7.46, \mathrm{~s}$ \\
\hline $\mathrm{HO}-4$ & & $15.93, \mathrm{~s}$ & & $14.49, \mathrm{~s}$ & & $14.30, \mathrm{~s}$ \\
\hline HO-9 & & $14.40, \mathrm{~s}$ & & $15.77, \mathrm{~s}$ & & 14.16 \\
\hline HO-11 & & $7.50, \mathrm{~s}$ & & & & $7.50, \mathrm{~s}$ \\
\hline HO-14 & & 3.82 , brs & & & & \\
\hline
\end{tabular}

${ }^{\text {a Recorded at }} 400 \mathrm{MHz}$ for ${ }^{1} \mathrm{H}$ and $100 \mathrm{MHz}$ for ${ }^{13} \mathrm{C}\left\{{ }^{1} \mathrm{H}\right\}$.

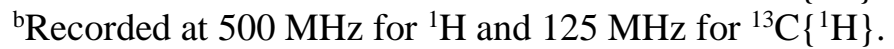

${ }^{\text {cRecorded at }} 700 \mathrm{MHz}$ for ${ }^{1} \mathrm{H}$ and $175 \mathrm{MHz}$ for ${ }^{13} \mathrm{C}\left\{{ }^{1} \mathrm{H}\right\}$. 

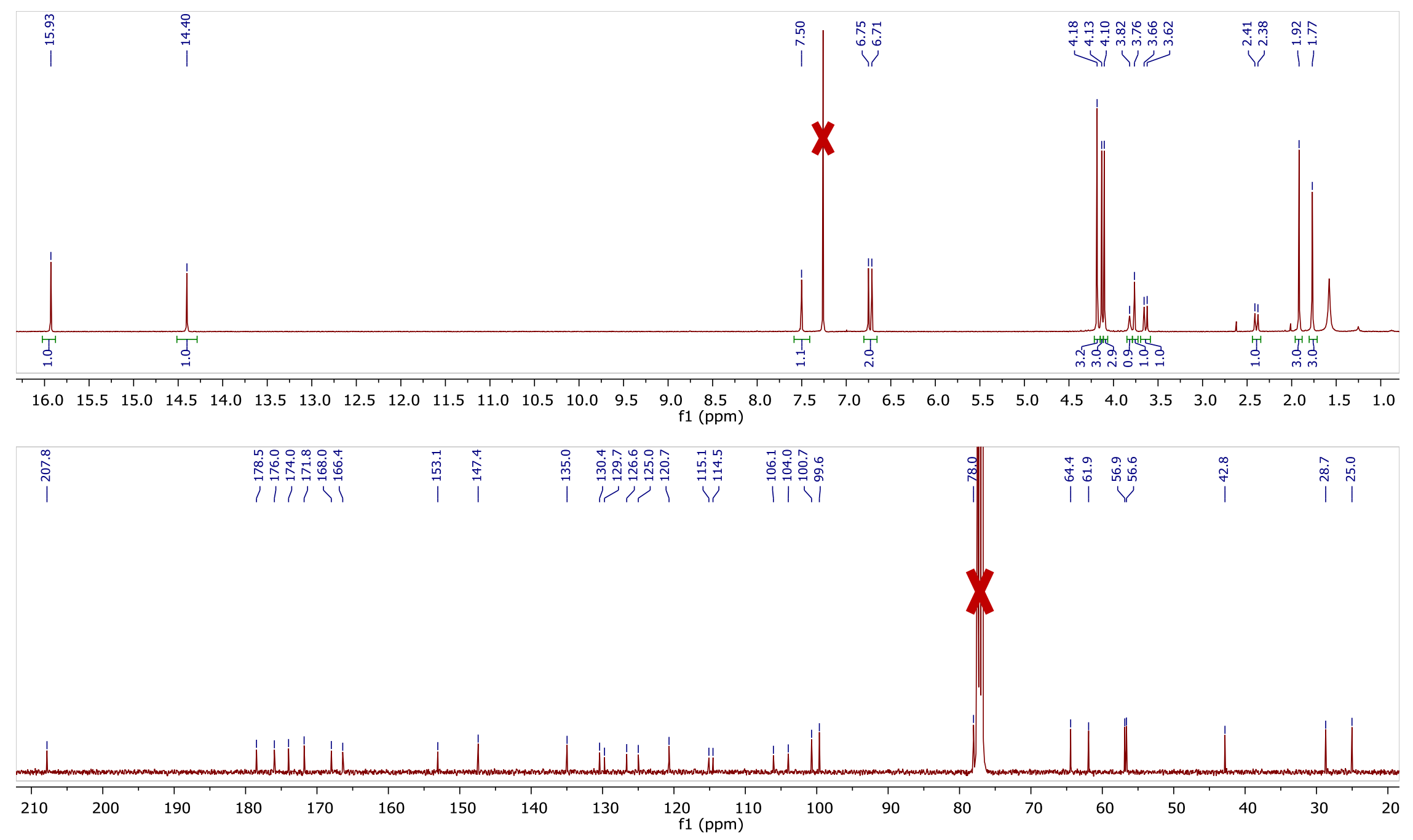

Figure S15. ${ }^{1} \mathrm{H}$ - and ${ }^{13} \mathrm{C}\left\{{ }^{1} \mathrm{H}\right\}$-NMR spectra of 8 [400 MHz for ${ }^{1} \mathrm{H}$ and $100 \mathrm{MHz}$ for $\left.{ }^{13} \mathrm{C}\left\{{ }^{1} \mathrm{H}\right\}, \mathrm{CDCl}_{3}\right]$. 

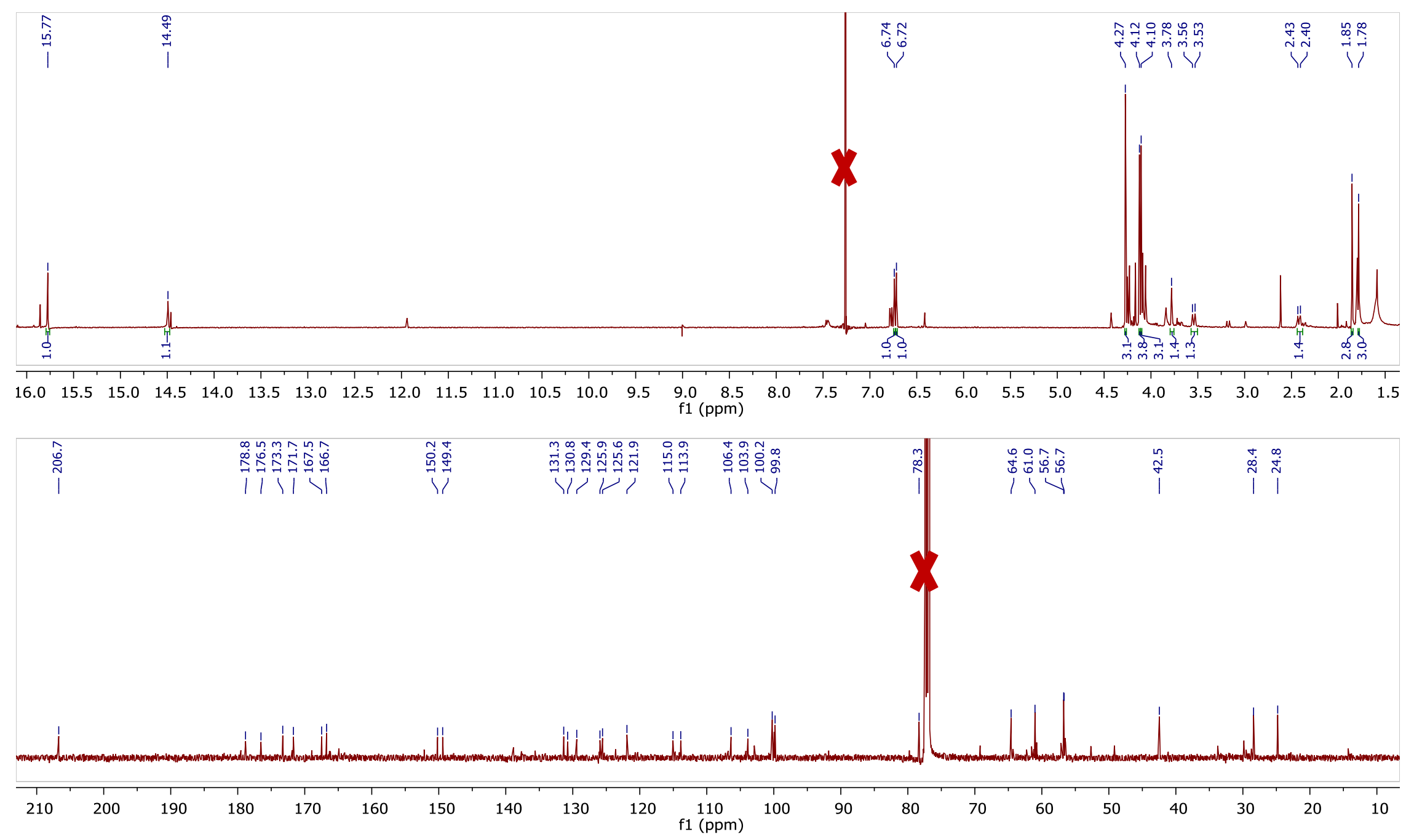

Figure S16. ${ }^{1} \mathrm{H}$ - and ${ }^{13} \mathrm{C}\left\{{ }^{1} \mathrm{H}\right\}$-NMR spectra of 9 [500 MHz for ${ }^{1} \mathrm{H}$ and $125 \mathrm{MHz}$ for $\left.{ }^{13} \mathrm{C}\left\{{ }^{1} \mathrm{H}\right\}, \mathrm{CDCl}_{3}\right]$. 

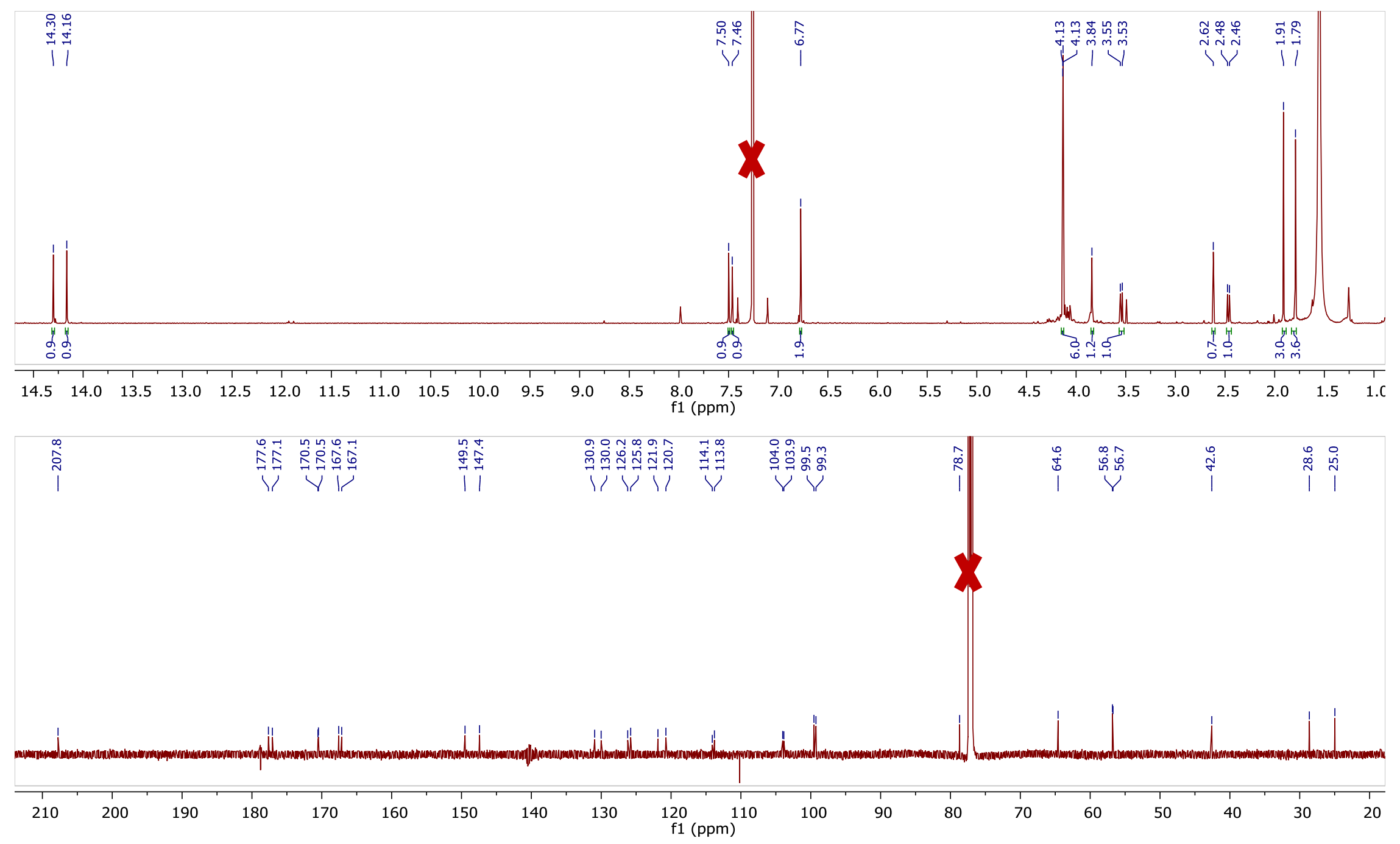

Figure S17. ${ }^{1} \mathrm{H}$ - and ${ }^{13} \mathrm{C}\left\{{ }^{1} \mathrm{H}\right\}$-NMR spectra of $\mathbf{1 0}\left[700 \mathrm{MHz}\right.$ for ${ }^{1} \mathrm{H}$ and $175 \mathrm{MHz}$ for $\left.{ }^{13} \mathrm{C}\left\{{ }^{1} \mathrm{H}\right\}, \mathrm{CDCl}_{3}\right]$. 

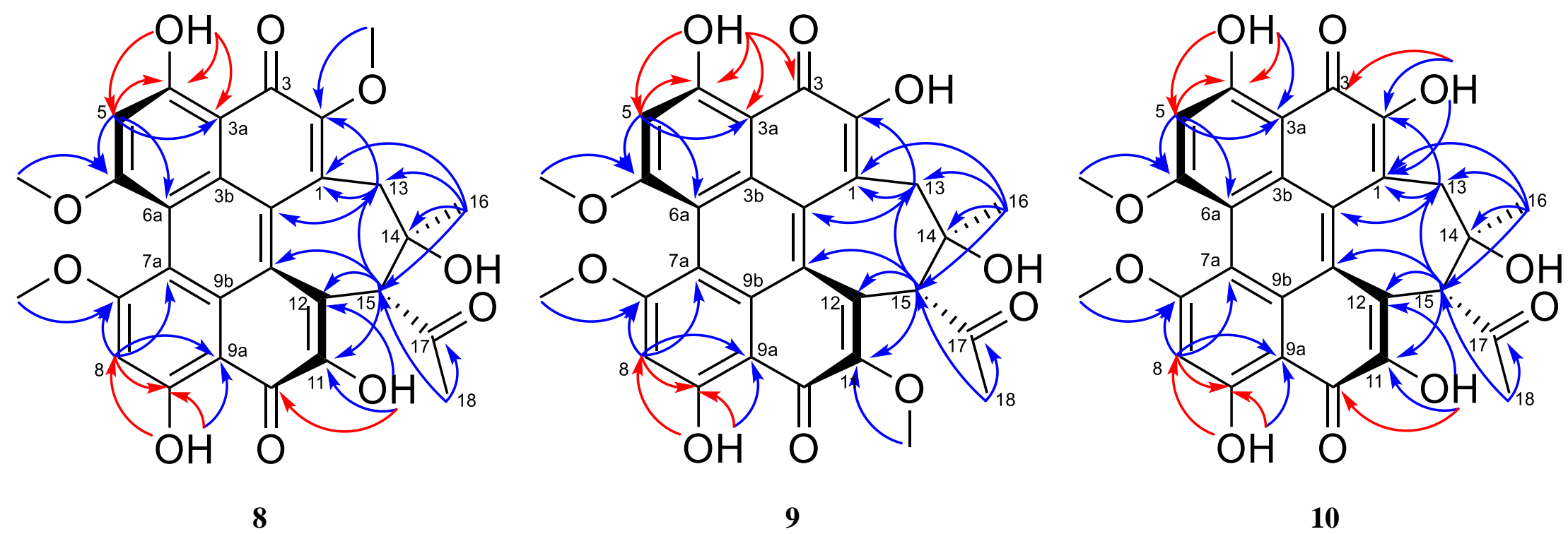

Figure S18. Key HMBC correlations for compounds 8-10. Arrows highlighted in red confirm the tautomeric structures of 8-10. 


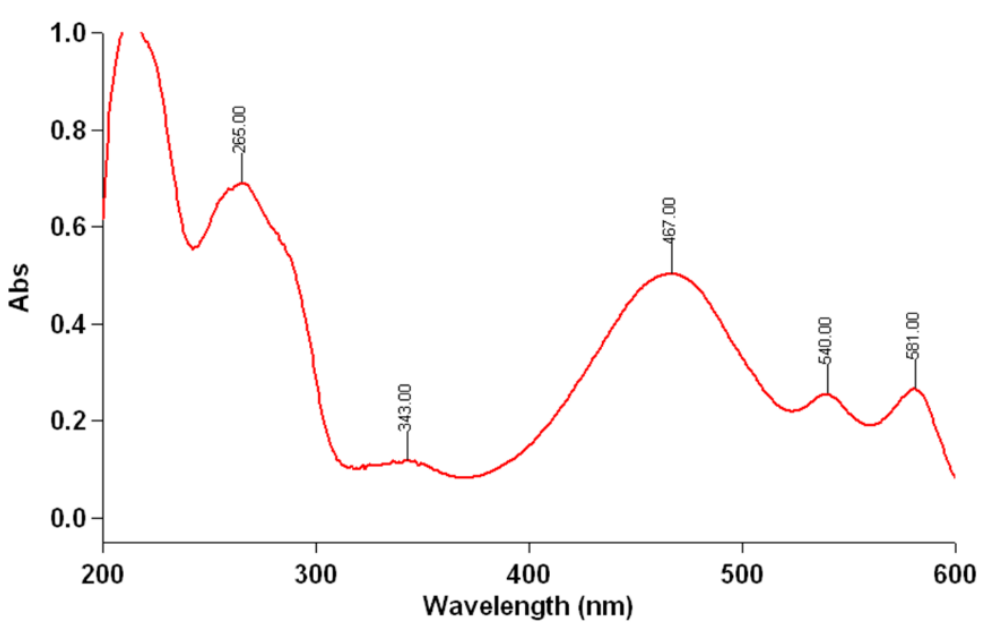

ent-shiraiachrome A (1)

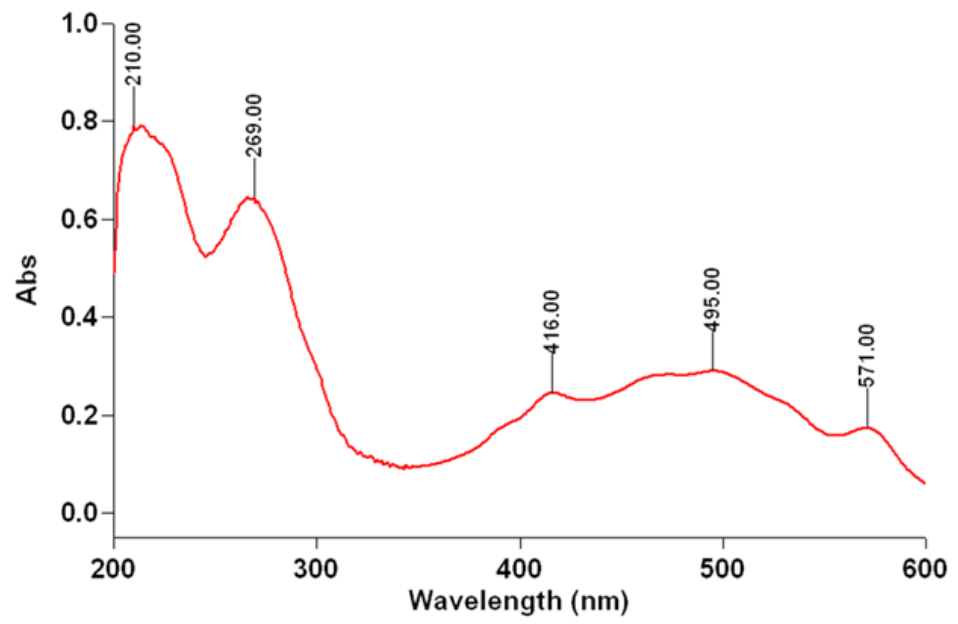

(9)



(8)

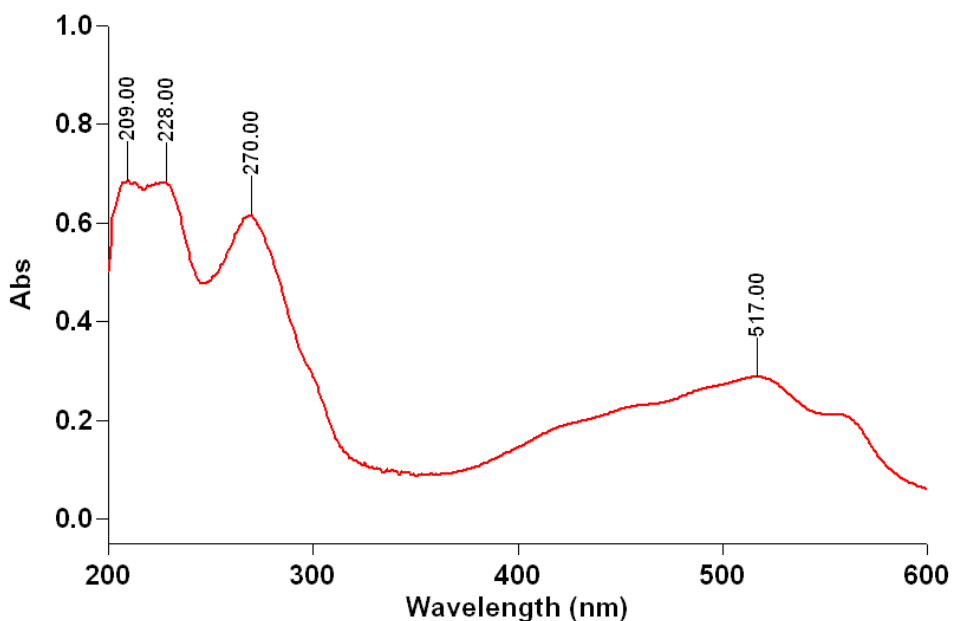

(10)

Figure S19. UV-vis spectra of compounds $\mathbf{1}$ and 8-10 in methanol at concentration of $0.0125 \mathrm{mg} / \mathrm{mL}$. 

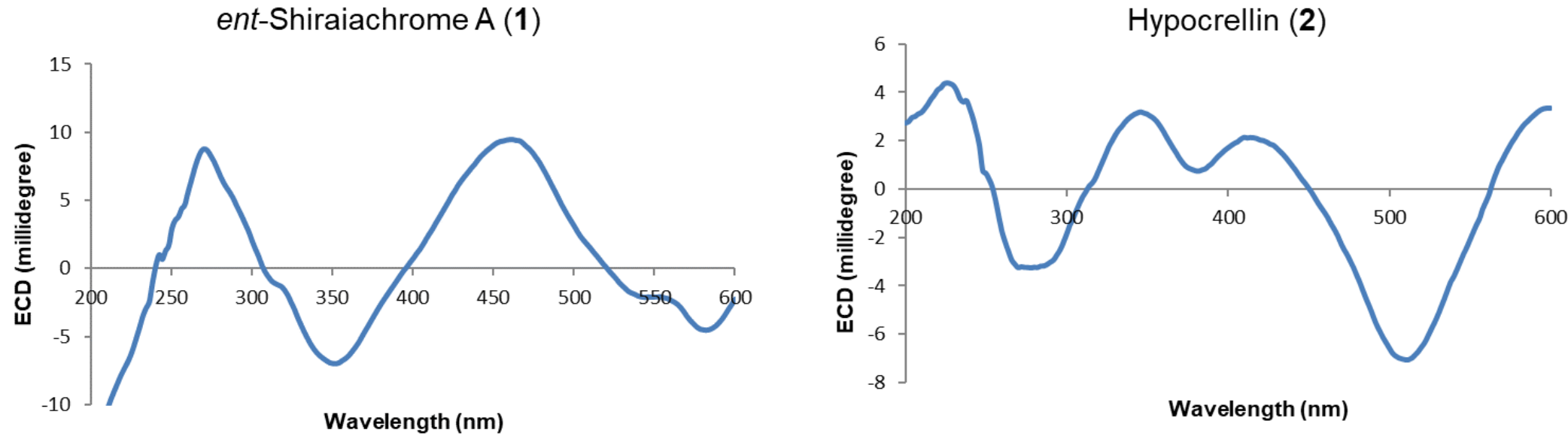

Hypocrellin B (3)
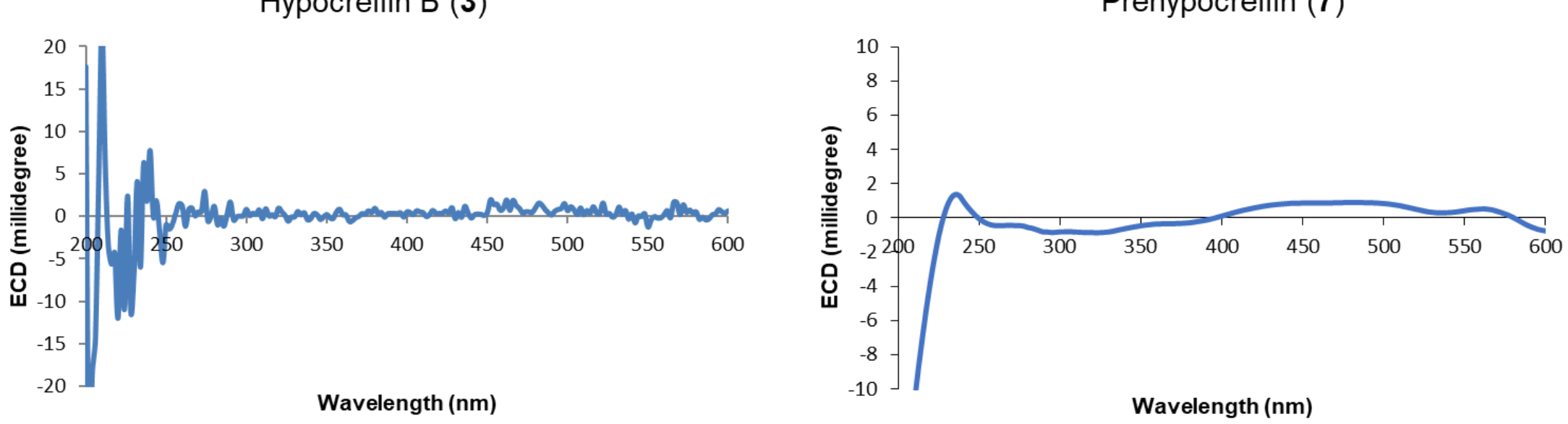

Figure S20. ECD spectra for compounds 1-3 and 7 in $\mathrm{CH}_{3} \mathrm{OH}$ at a concentration of $0.2 \mathrm{mg} / \mathrm{mL}$. 

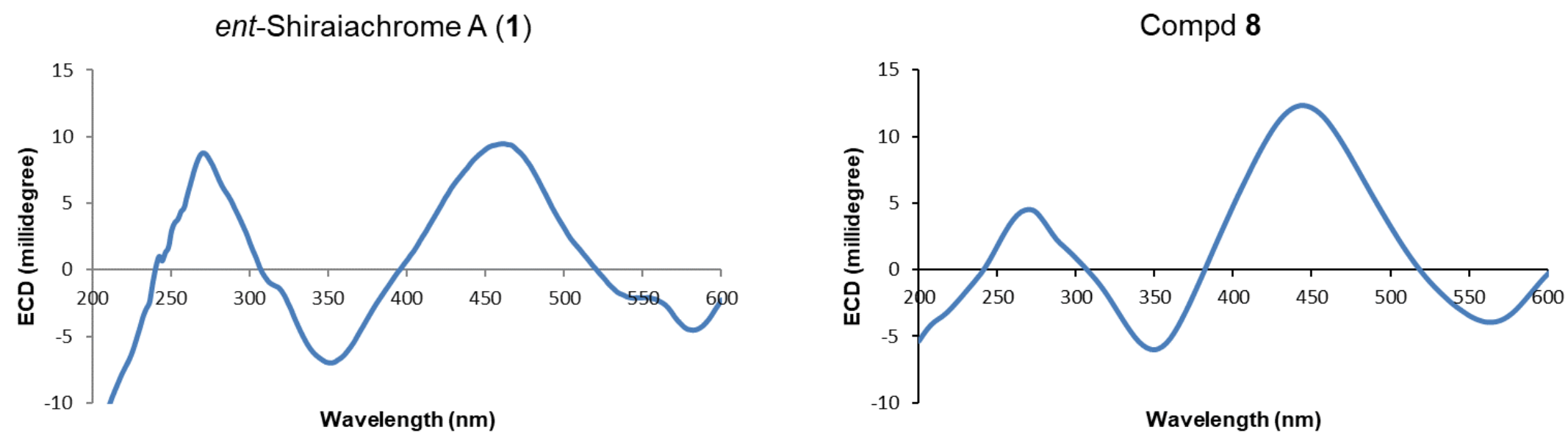

Compd 9
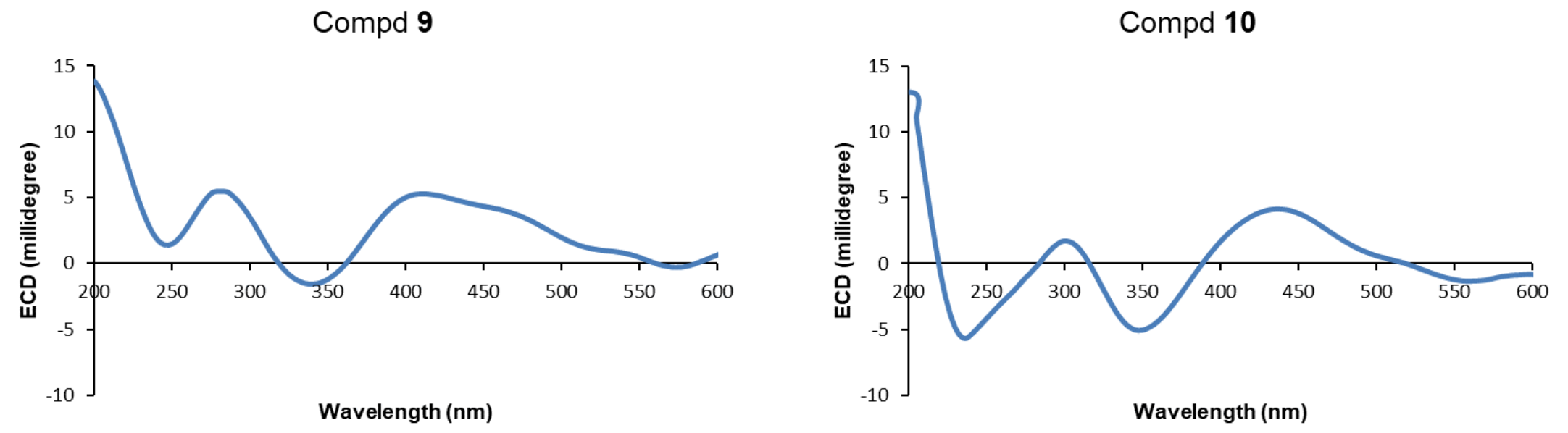

Figure S21. ECD spectra for compounds 8-10 as compared to ent-shiraiachrome A (1) in $\mathrm{CH}_{3} \mathrm{OH}$ at a concentration of $0.2 \mathrm{mg} / \mathrm{mL}$. 
Hypomycin A (4)

$\mathrm{C}_{30} \mathrm{H}_{28} \mathrm{O}_{10}, 549.17334$

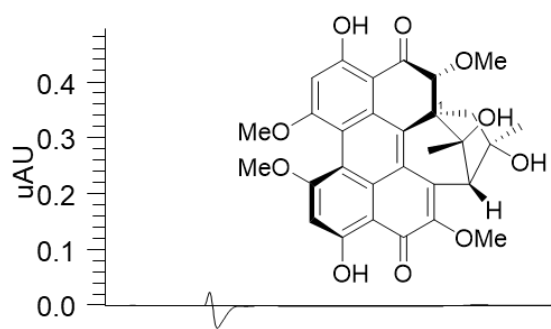

Before treatment with $\mathrm{Na}_{2} \mathrm{~S}_{2} \mathrm{O}_{4}$

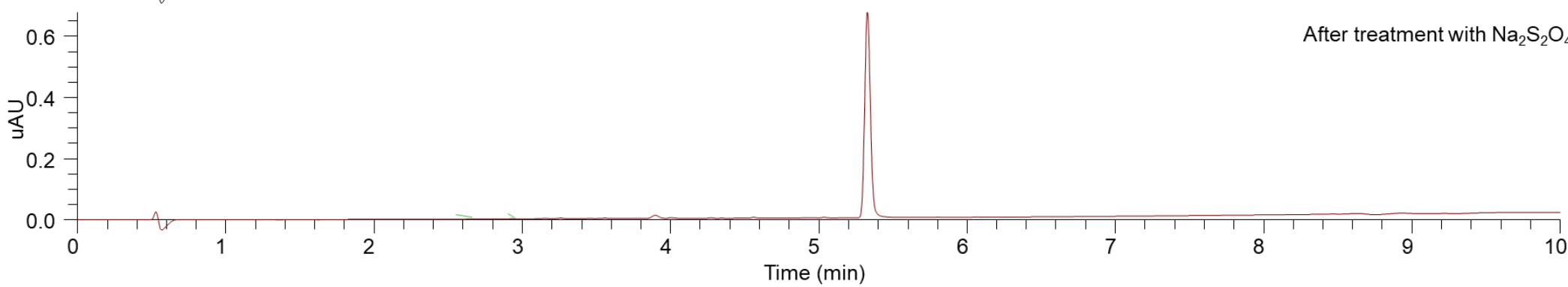

Figure S22. UPLC-HRESIMS chromatogram of hypomycin A (4) before and after treatment with $\mathrm{Na}_{2} \mathrm{~S}_{2} \mathrm{O}_{4}$ as a reducing agent overnight. 


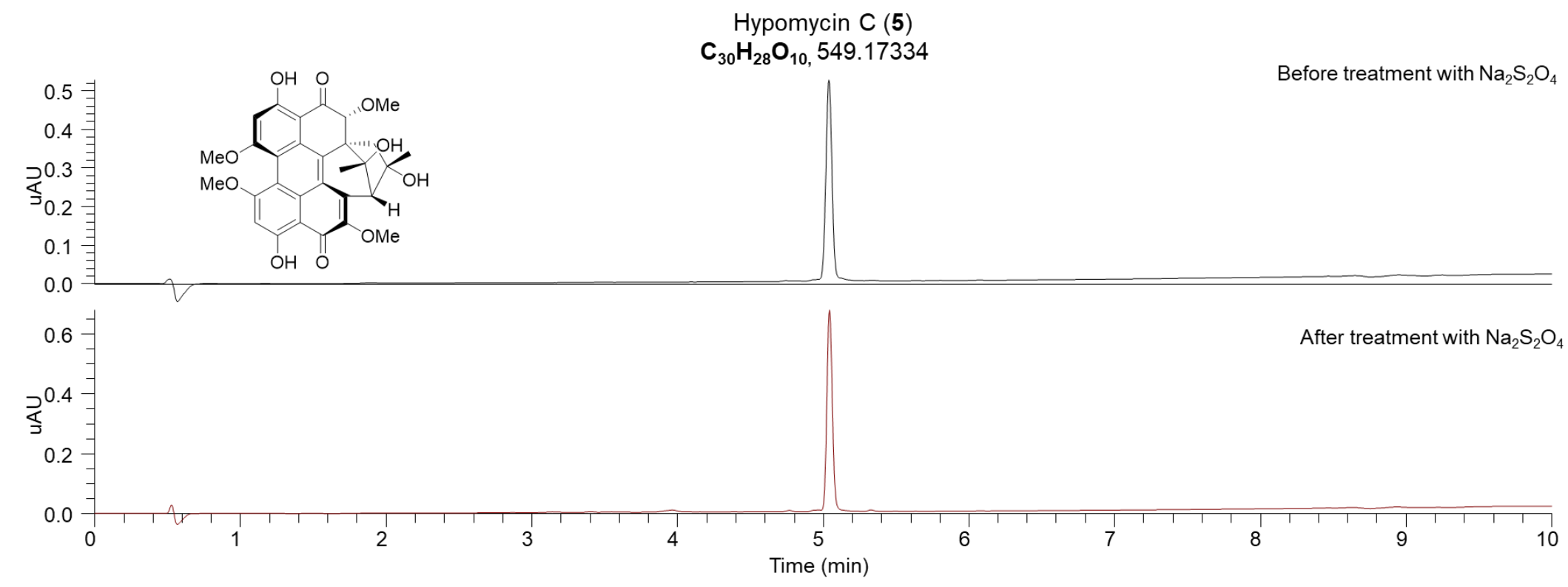

Figure S23. UPLC-HRESIMS chromatogram of hypomycin C (5) before and after treatment with $\mathrm{Na}_{2} \mathrm{~S}_{2} \mathrm{O}_{4}$ as a reducing agent overnight. 


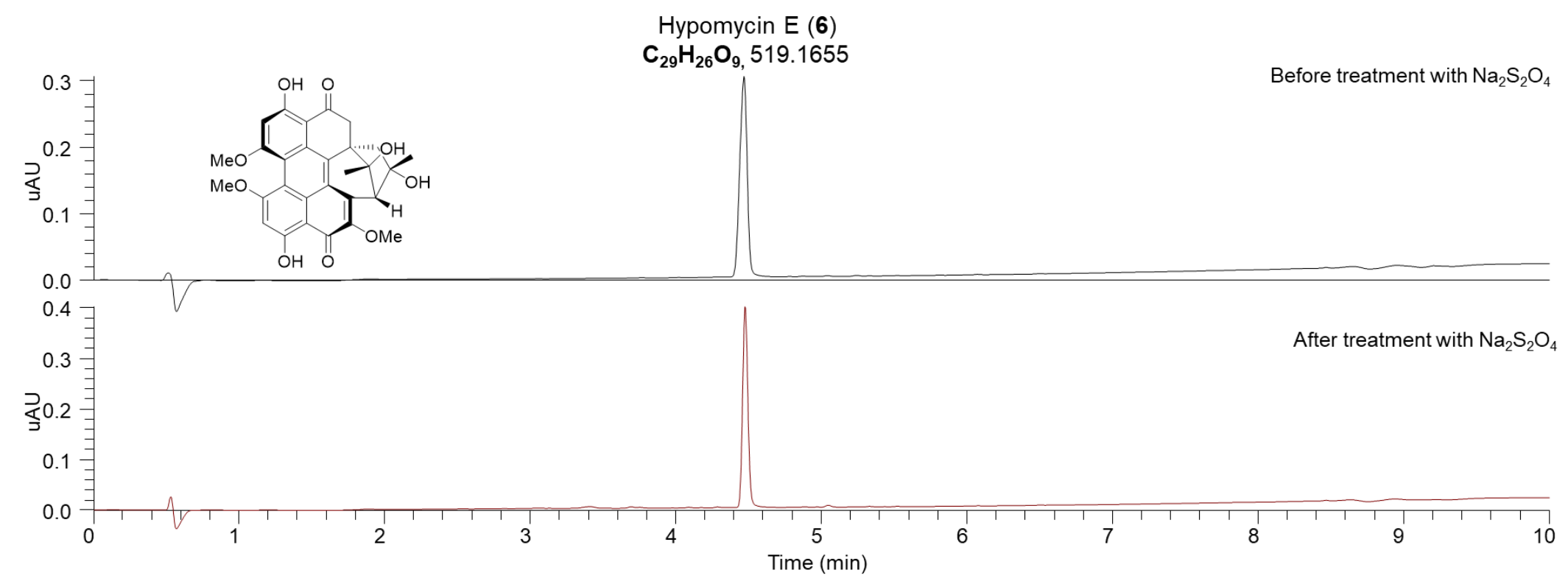

Figure S24. UPLC-HRESIMS chromatogram of hypomycin E (6) before and after treatment with $\mathrm{Na}_{2} \mathrm{~S}_{2} \mathrm{O}_{4}$ as a reducing agent overnight. 



$0.5 \mathrm{mg} /$ compound

Figure S25. Small scale reduction reactions of hypomycin A (4), C (5), and E (6) with $\mathrm{Na}_{2} \mathrm{~S}_{2} \mathrm{O}_{4}$ under aerobic conditions. Note the absence of a color change, relative to what was observed for 1-3 (Figure S9). 

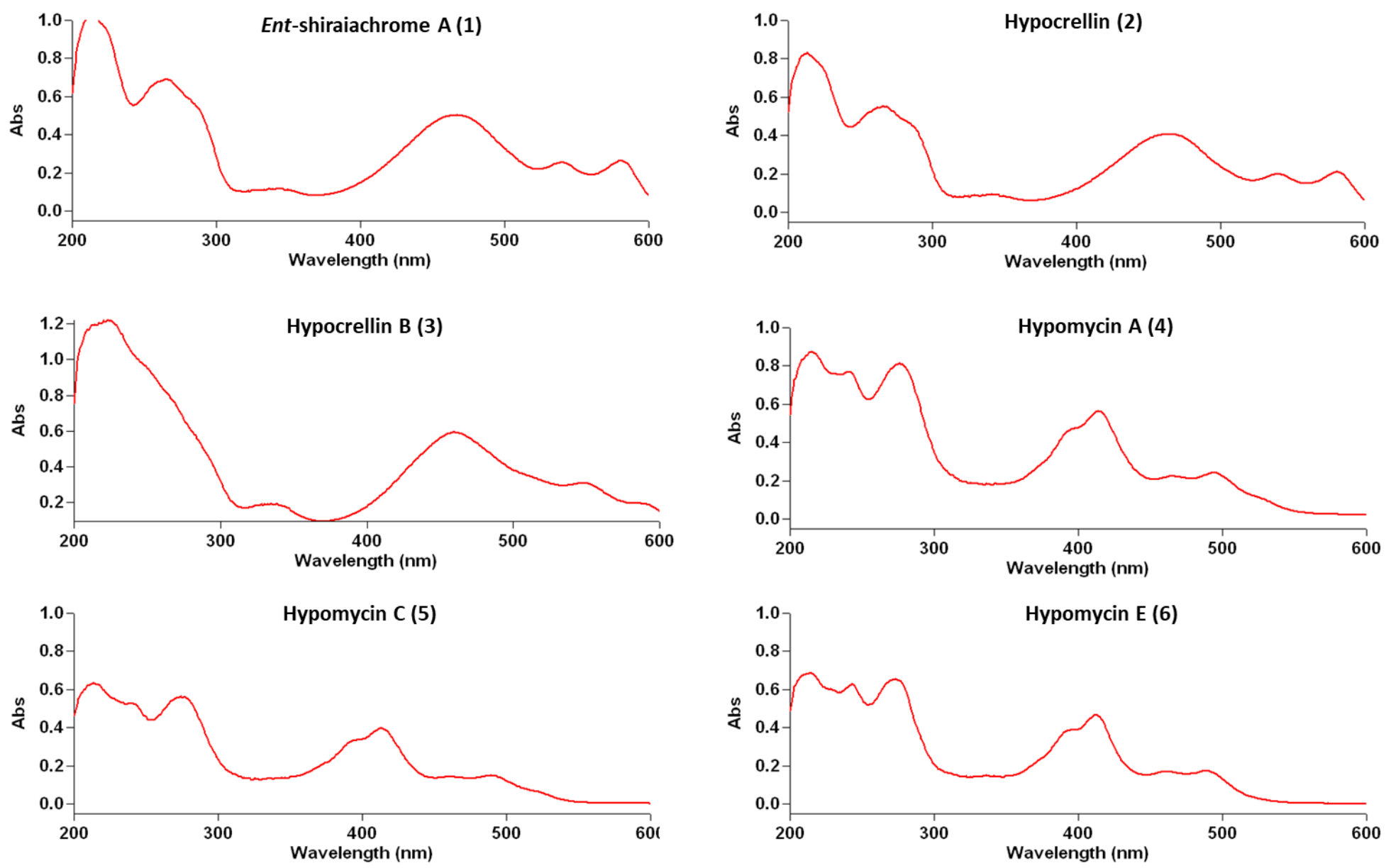

Figure S26. UV-vis spectra of compounds 1-6 in methanol at concentration of $0.0125 \mathrm{mg} / \mathrm{mL}$. 


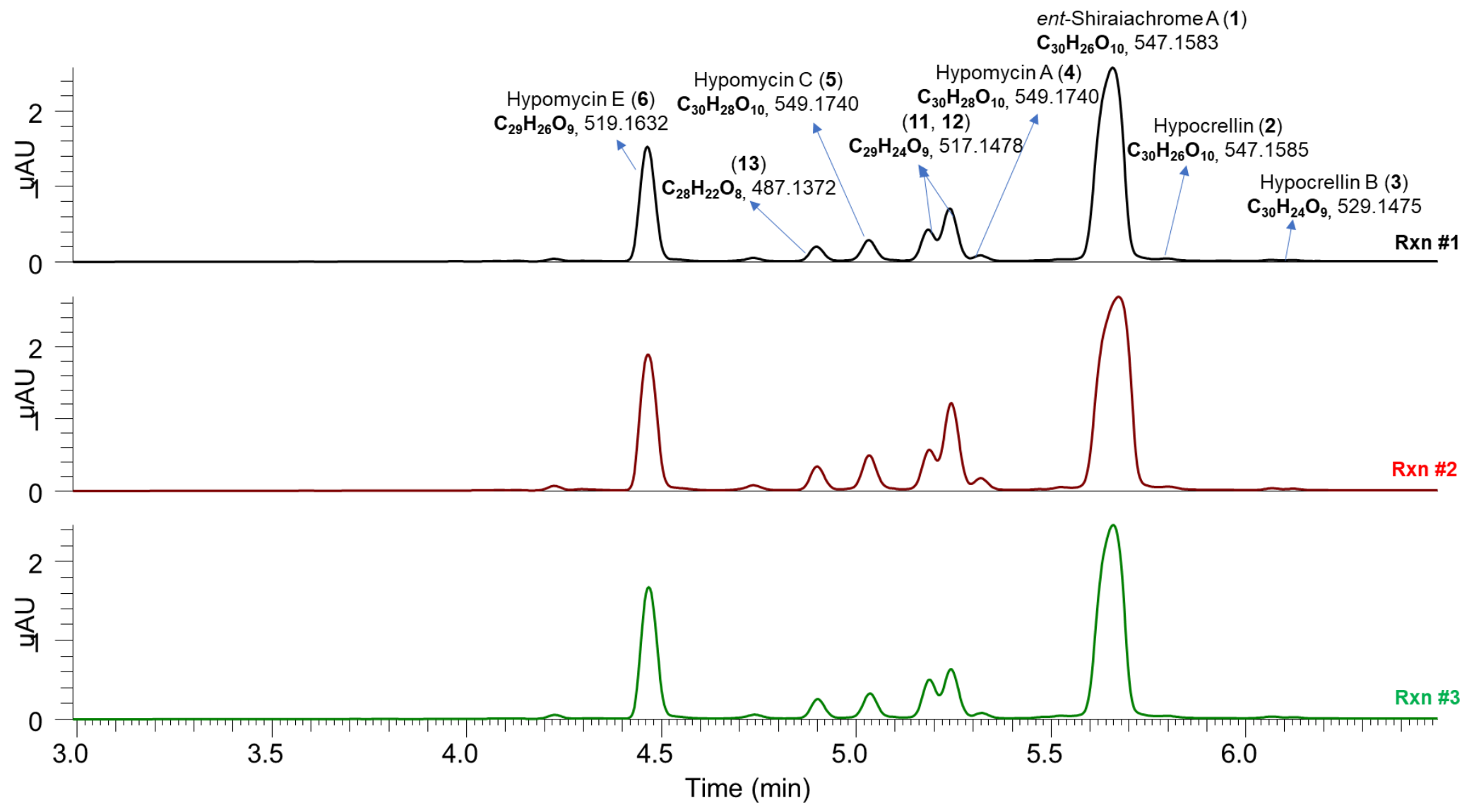

Figure S27. UPLC-HRESIMS chromatograms for the triplicate anaerobic reduction reactions of ent-shiraiachrome A (1) with $\mathrm{Na}_{2} \mathrm{~S}_{2} \mathrm{O}_{4}\left(10 \mathrm{mg}\right.$ of $\mathbf{1}$ was used in each reaction). HRESIMS data are reported as $[\mathrm{M}+\mathrm{H}]^{+}$. 


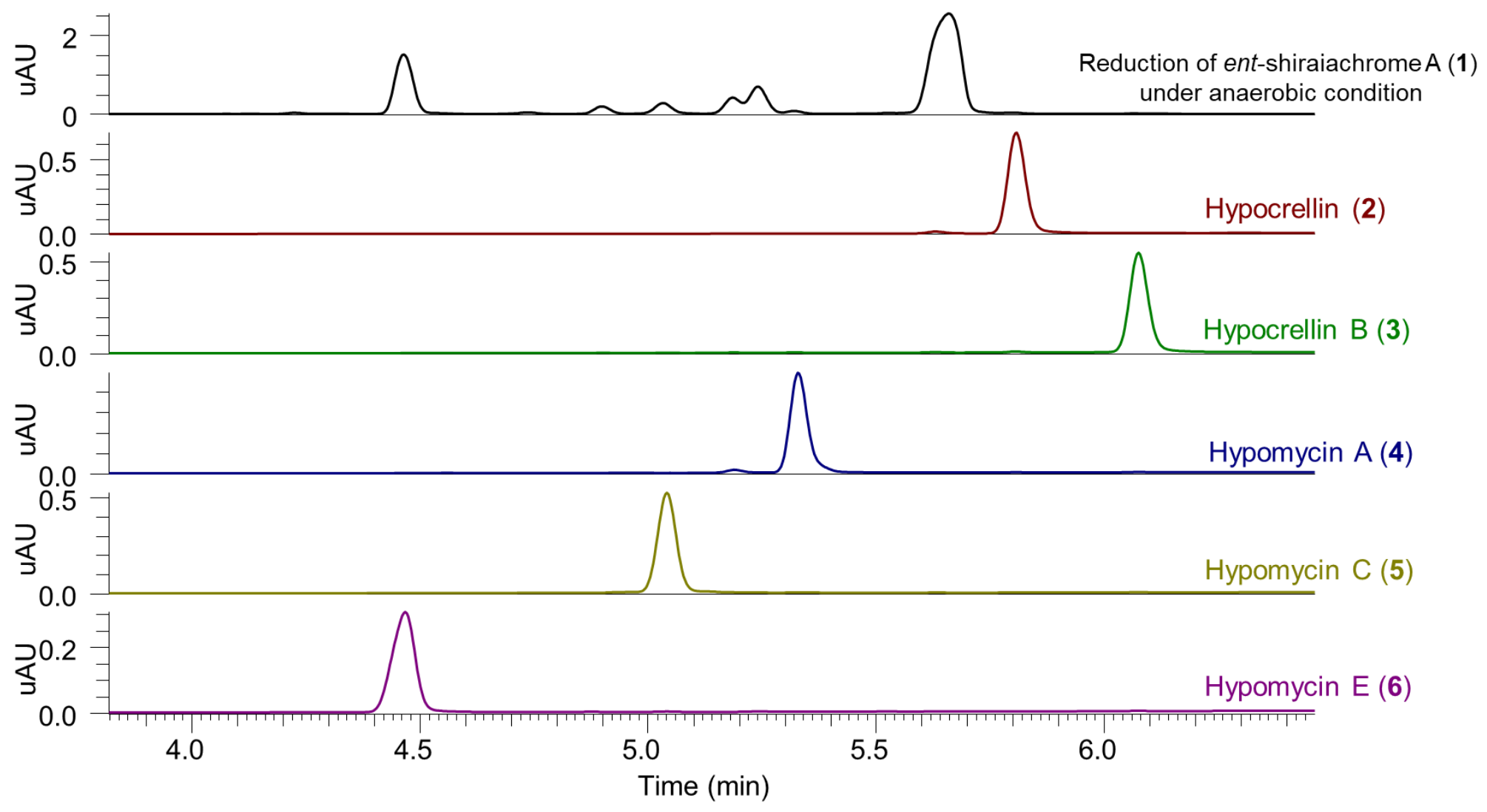

Figure S28. UPLC-HRESIMS chromatograms for the anaerobic reduction reactions of ent-shiraiachrome A (1) as compared to UPLC-HRESIMS chromatograms of pure standards of 2-6. 
Hypomycin A (4)

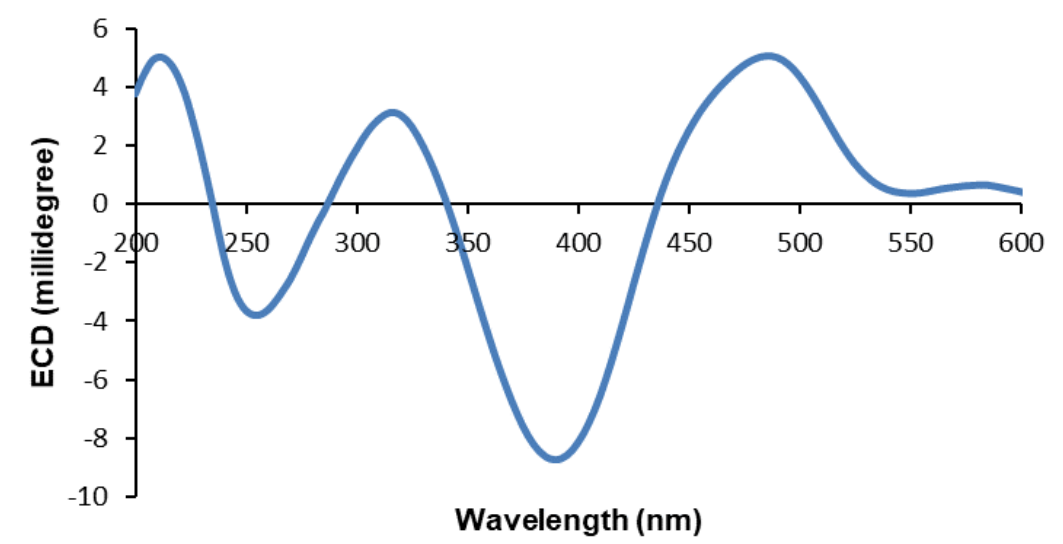

Hypomycin E (6)

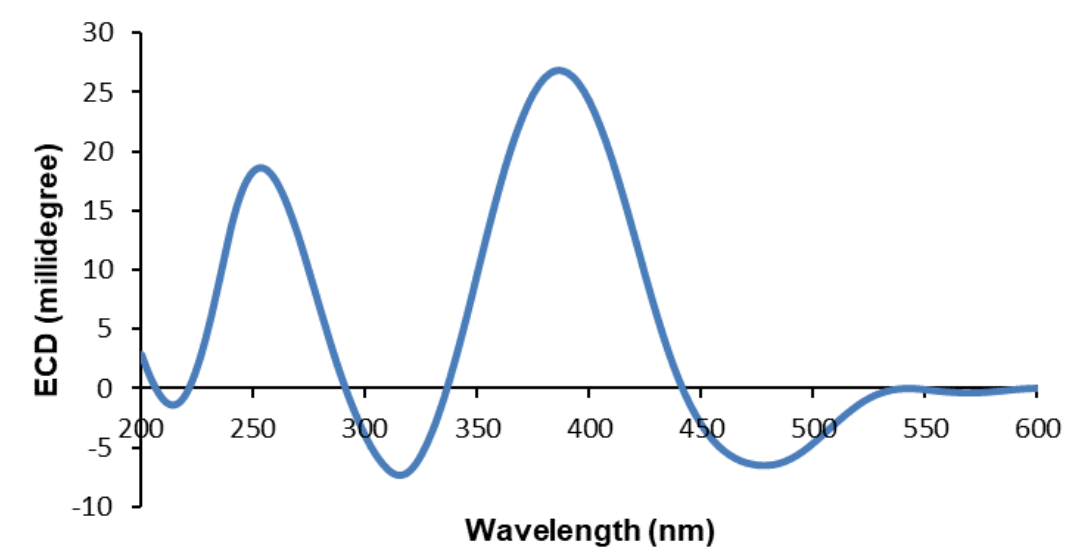

Hypomycin C (5)

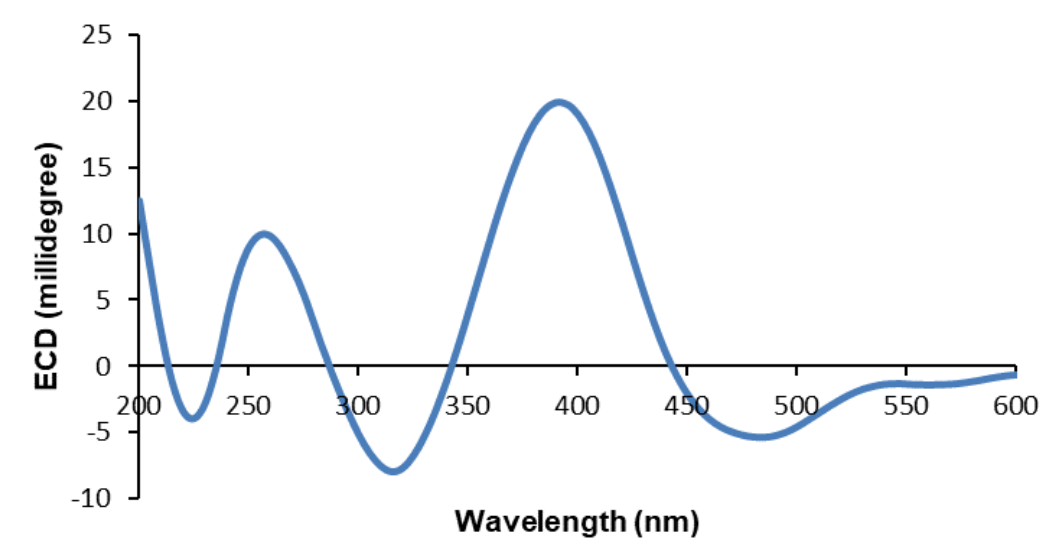

Compd (11)

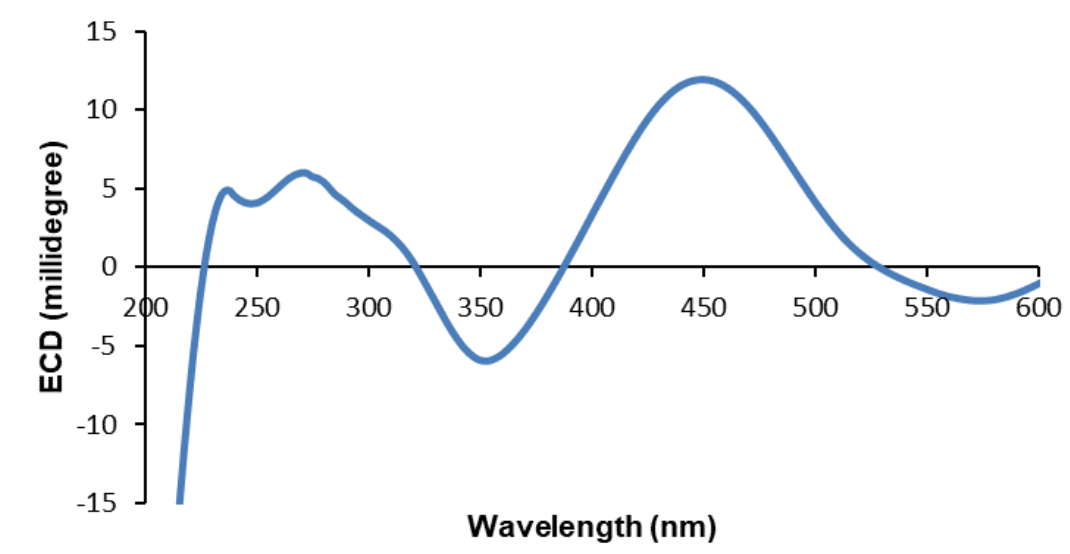

Figure S29. ECD spectra in $\mathrm{CH}_{3} \mathrm{OH}$ for compounds 4-6 and 11 which were isolated from the anaerobic reduction reaction of $\mathbf{1}$ and 2. 

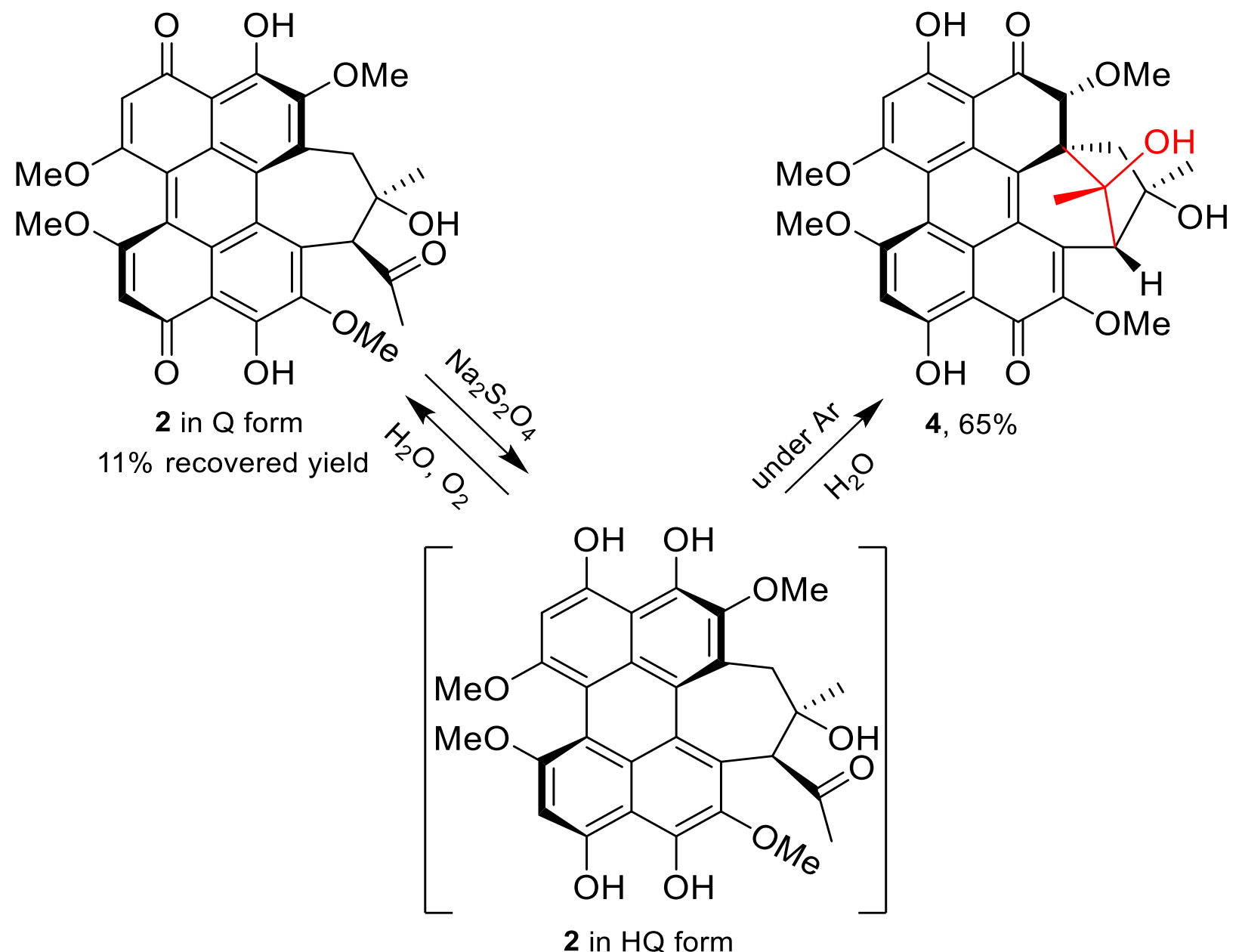

Figure S30. Proposed generation of hypomycin A (4) via reduction of hypocrellin (2) with $\mathrm{Na}_{2} \mathrm{~S}_{2} \mathrm{O}_{4}$ under anaerobic conditions. 
A ${ }^{1} \mathrm{H}$ NMR of pure ent-shiraiachrome A (1) sample in DMSO- $d_{6}$

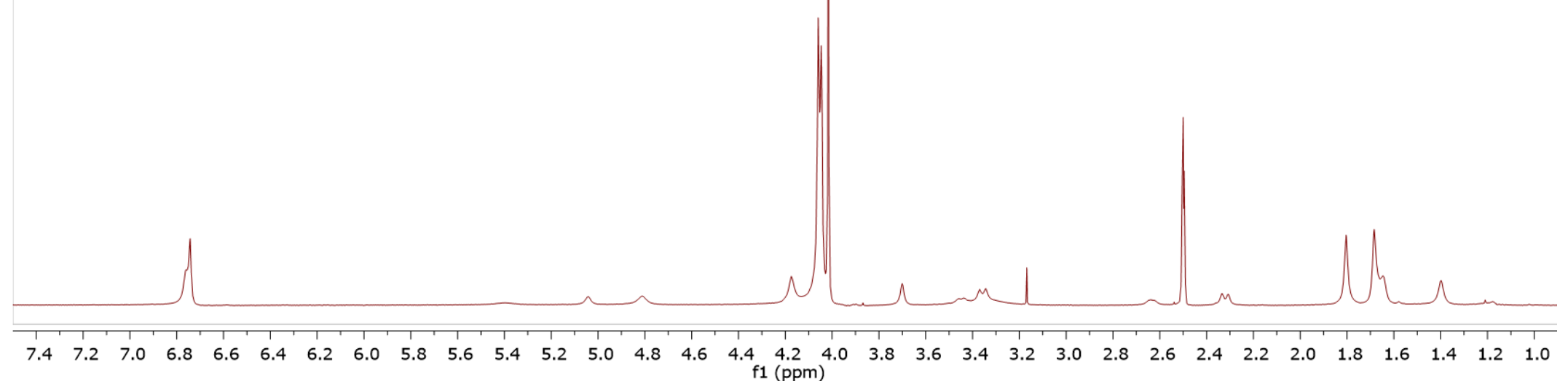

$\mathbf{B}$

${ }^{1} \mathrm{H}$ NMR of pure ent-shiraiachrome A (1) sample in $\mathrm{CDCl}_{3}$ after exposure to DMSO

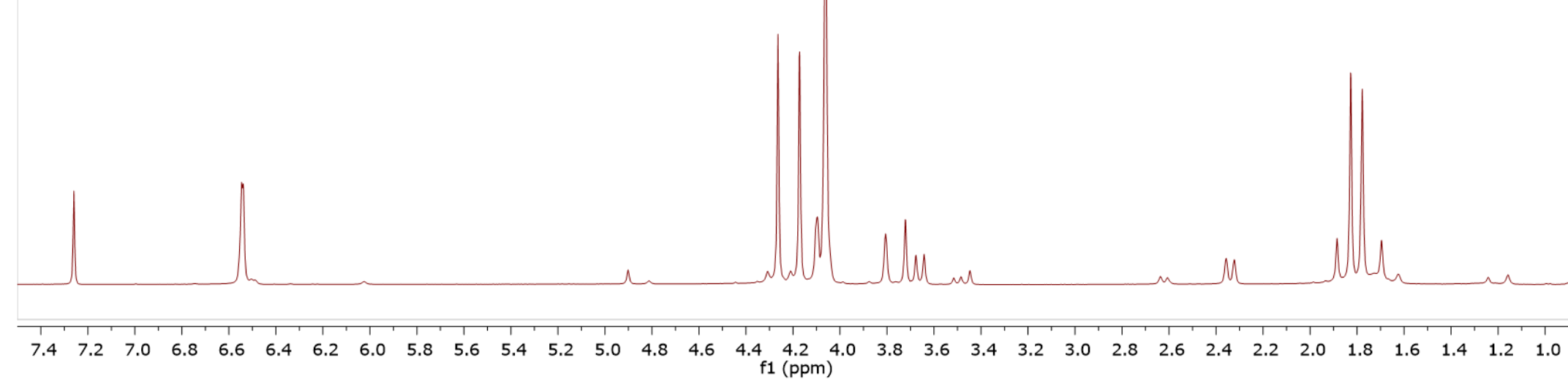

Figure S31. ${ }^{1} \mathrm{H}-\mathrm{NMR}$ of ent-shiraiachrome A (1) in DMSO- $d_{6}(\mathbf{A})$ and ${ }^{1} \mathrm{H}-\mathrm{NMR}$ of $\mathbf{1}$ in $\mathrm{CDCl}_{3}$ after exposure to DMSO- $d_{6}(\mathbf{B})$. 


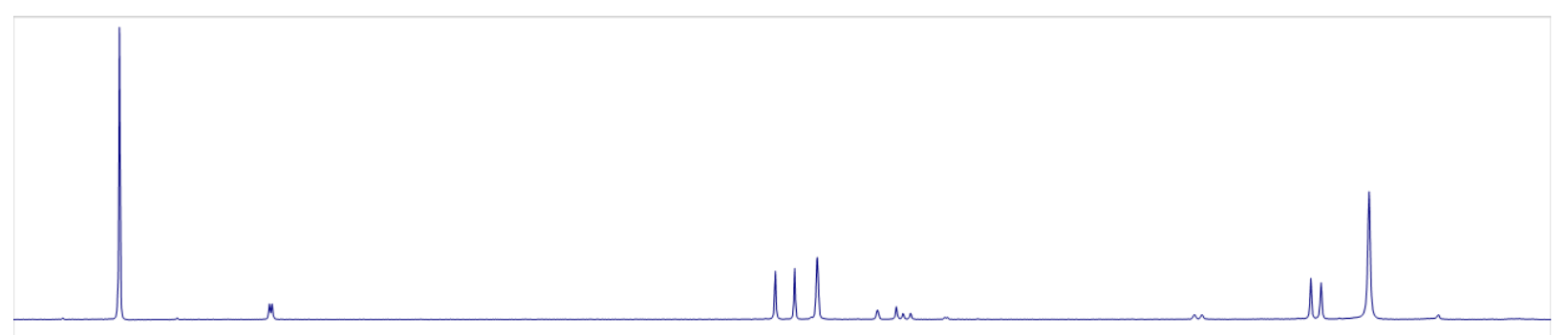

${ }^{1} \mathrm{H}$ NMR of entshiraiachrome A (1) in $\mathrm{CDCl}_{3}$ before exposure to DMSO

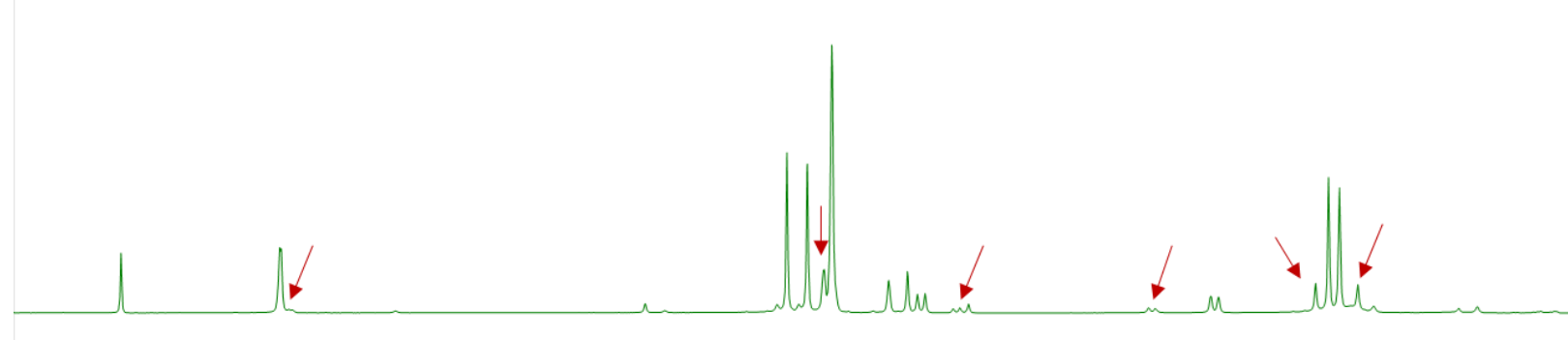

\section{${ }^{1} \mathrm{H}$ NMR of ent-} shiraiachrome A sample in $\mathrm{CDCl}_{3}$ after exposure to DMSO

\section{${ }^{1} \mathrm{H}$ NMR of} hypocrellin (2) A

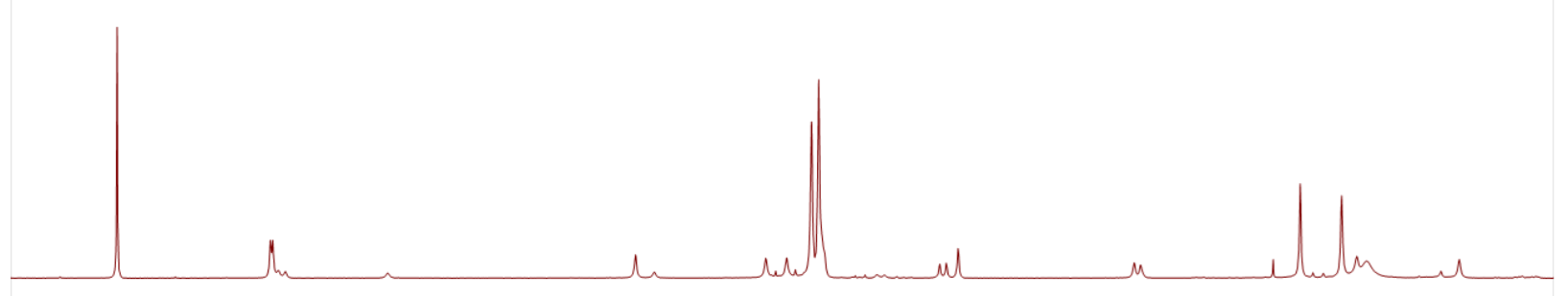
in $\mathrm{CDCl}_{3}$

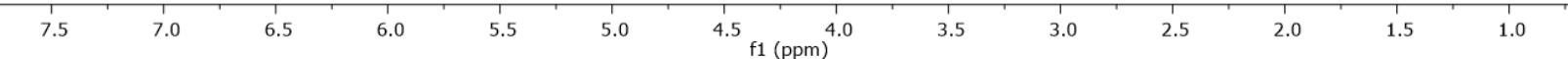

Figure S32. ${ }^{1} \mathrm{H}-\mathrm{NMR}$ of pure ent-shiraiachrome A (1) in $\mathrm{CDCl}_{3}$ before and after exposure to DMSO vs ${ }^{1} \mathrm{H}-\mathrm{NMR}$ of hypocrellin (2) in $\mathrm{CDCl}_{3}$. Red arrows show the new set on peaks formed in the ent-shiraiachrome A (1) sample after exposure to DMSO, which matches the ${ }^{1} \mathrm{H}-\mathrm{NMR}$ of hypocrellin (2). 


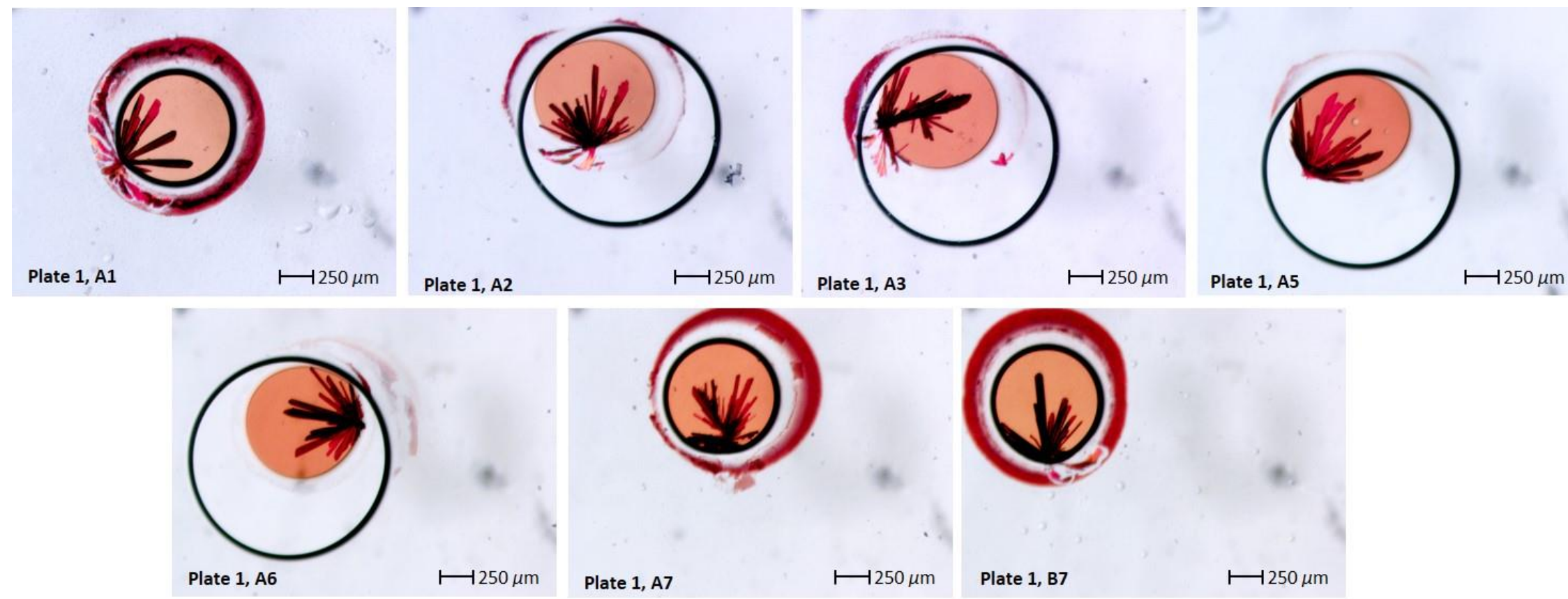

Figure S33. Typical single crystals of hypocrellin B (3) suitable for X-ray diffraction analysis, prepared via ENaCt after 14 days. 


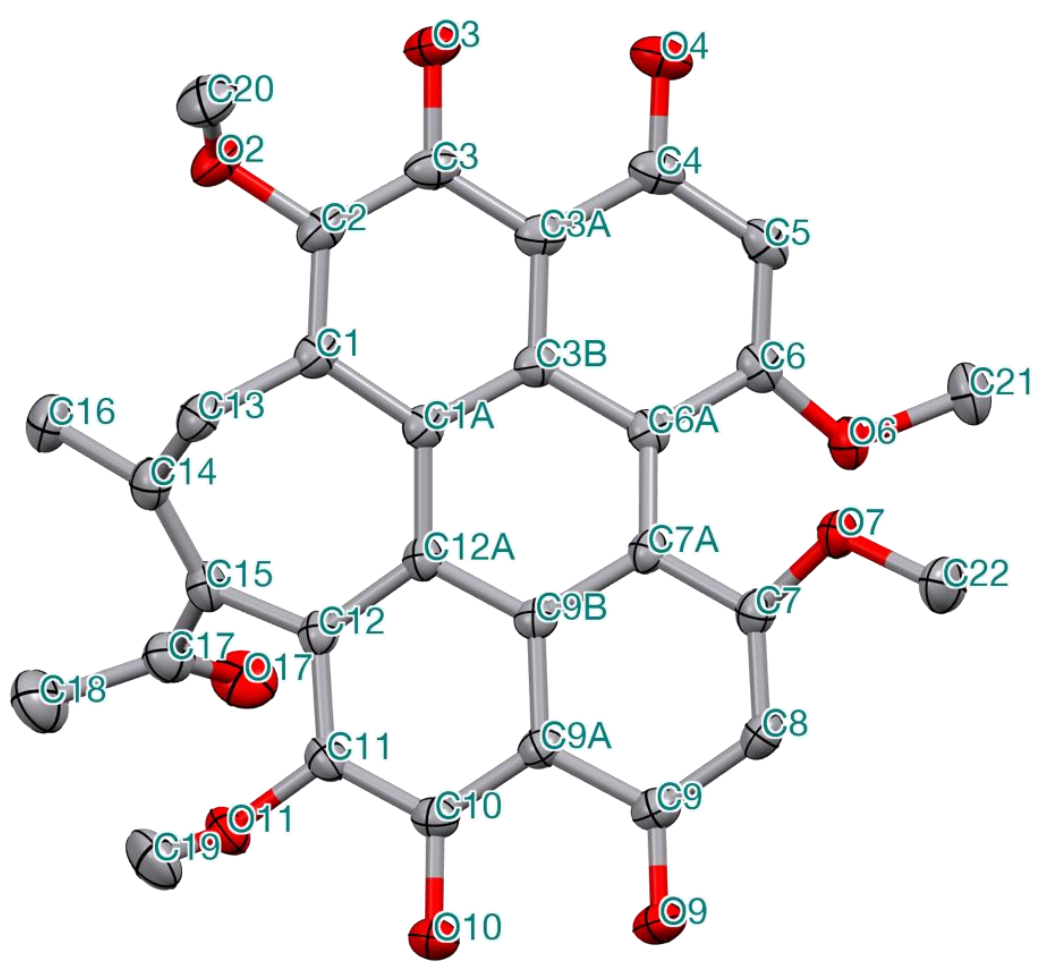

Figure S34. Displacement ellipsoid plot (50\% probability level) of hypocrellin B (3), showing the atomlabelling scheme. Hydrogen atoms and solvent molecule have been omitted for clarity. 
Table S5. Crystal data and structure refinement for hypocrellin B (3).

\begin{tabular}{|c|c|}
\hline Identification code & klh_01084_45_1_p1_b7_0m_face_s \\
\hline Empirical formula & $\mathrm{C}_{32} \mathrm{H}_{30} \mathrm{O}_{10} \mathrm{~S}$ \\
\hline Formula weight & 606.62 \\
\hline Temperature/K & 150.0 \\
\hline Crystal system & orthorhombic \\
\hline Space group & Pna 21 \\
\hline $\mathrm{a} / \AA$ & $10.1873(4)$ \\
\hline $\mathrm{b} / \AA$ & $16.2677(6)$ \\
\hline $\mathrm{c} / \AA$ & $16.9005(6)$ \\
\hline$\alpha /^{\circ}$ & 90 \\
\hline$\beta /{ }^{\circ}$ & 90 \\
\hline$\gamma /{ }^{\circ}$ & 90 \\
\hline Volume $/ \AA^{3}$ & $2800.82(18)$ \\
\hline $\mathrm{Z}$ & 4 \\
\hline$\rho_{\text {calc }} \mathrm{g} / \mathrm{cm}^{3}$ & 1.439 \\
\hline$\mu / \mathrm{mm}^{-1}$ & 1.558 \\
\hline $\mathrm{F}(000)$ & 1272.0 \\
\hline Crystal size $/ \mathrm{mm}^{3}$ & $0.358 \times 0.08 \times 0.056$ \\
\hline Radiation & $\mathrm{CuK} \alpha(\lambda=1.54178)$ \\
\hline $2 \Theta$ range for data collection $/^{\circ}$ & 7.542 to 136.636 \\
\hline Index ranges & $-11 \leq \mathrm{h} \leq 12,-19 \leq \mathrm{k} \leq 18,-17 \leq 1 \leq 20$ \\
\hline Reflections collected & 18658 \\
\hline Independent reflections & $4834\left[\mathrm{R}_{\mathrm{int}}=0.0178, \mathrm{R}_{\text {sigma }}=0.0224\right]$ \\
\hline Data/restraints/parameters & $4834 / 1 / 404$ \\
\hline Goodness-of-fit on $\mathrm{F}^{2}$ & 1.052 \\
\hline Final $\mathrm{R}$ indexes $[\mathrm{I}>=2 \sigma(\mathrm{I})]$ & $\mathrm{R}_{1}=0.0249, \mathrm{wR}_{2}=0.0701$ \\
\hline Final $\mathrm{R}$ indexes [all data] & $\mathrm{R}_{1}=0.0251, \mathrm{wR}_{2}=0.0703$ \\
\hline Largest diff. peak/hole / e $\AA^{-3}$ & $0.17 /-0.26$ \\
\hline Flack parameter & $0.019(5)$ \\
\hline
\end{tabular}


${ }^{1} \mathrm{H}-\mathrm{NMR}$ for the anaerobic reduction reaction of $\mathbf{1}$ in acetonitrile- $\mathrm{d}_{3}$.

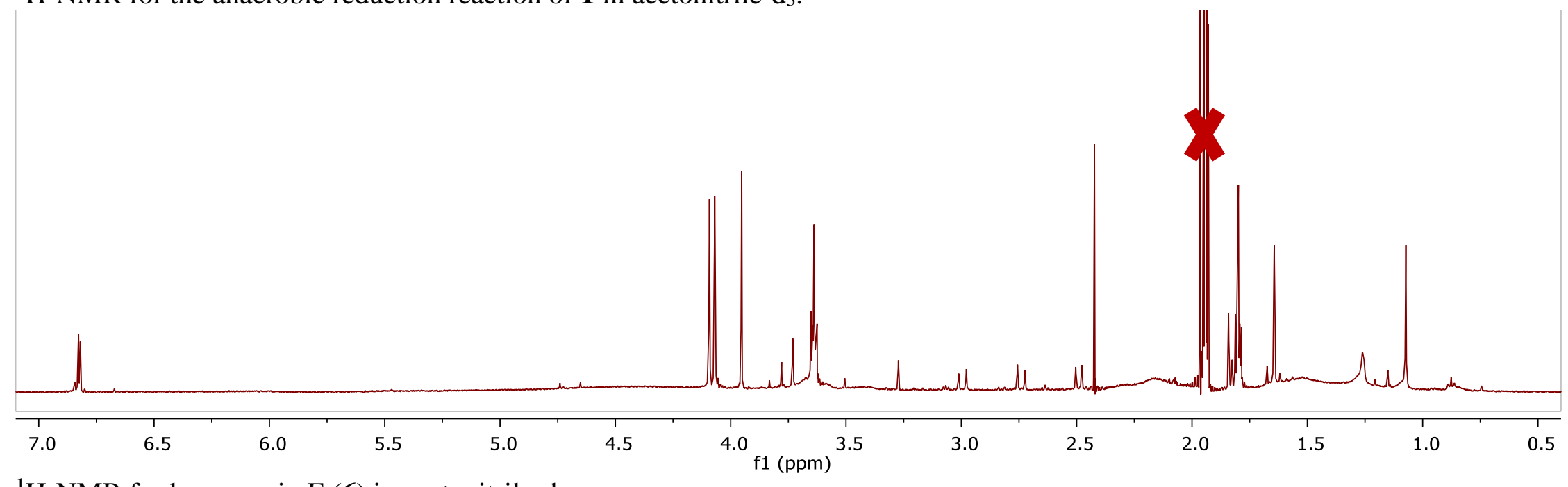

${ }^{1} \mathrm{H}-\mathrm{NMR}$ for hypomycin $\mathrm{E}(\mathbf{6})$ in acetonitrile- $\mathrm{d}_{3}$.

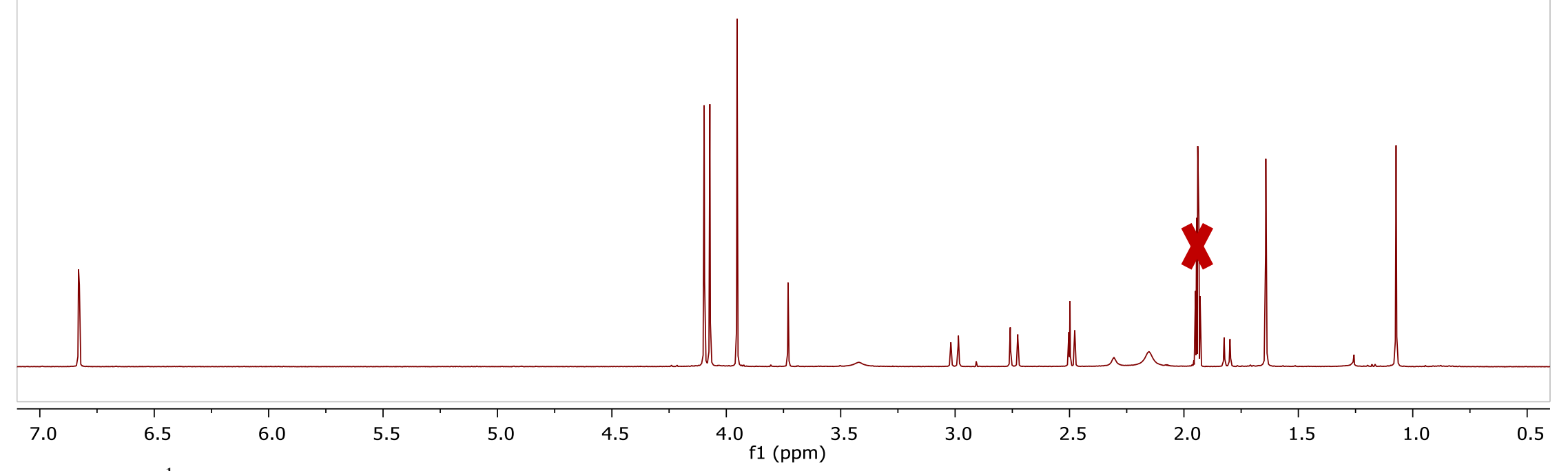

Figure S35. ${ }^{1} \mathrm{H}-\mathrm{NMR}$ for the anaerobic reduction reaction mixture of ent-shiraiachrome A (1) as compared to hypomycin E (6) in acetonitrile- $d_{3}$. 
${ }^{1} \mathrm{H}-\mathrm{NMR}$ for the anaerobic reduction reaction of $\mathbf{2}$ in acetonitrile- $d_{3}$.



${ }^{1} \mathrm{H}-\mathrm{NMR}$ for hypomycin A (4) in acetonitrile- $d_{3}$.

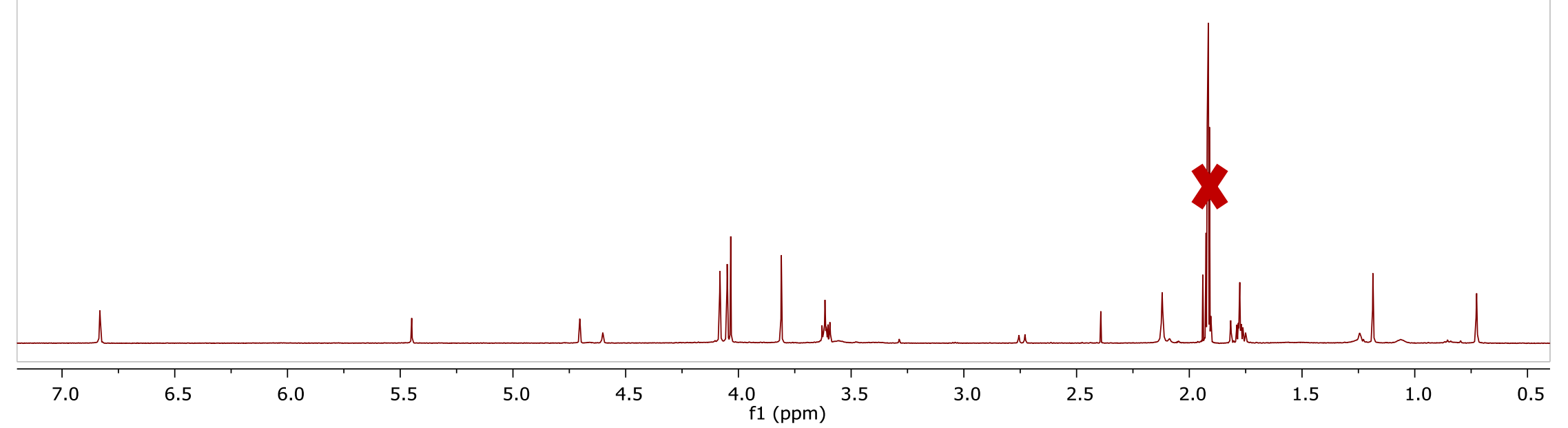

Figure S36. ${ }^{1} \mathrm{H}-\mathrm{NMR}$ for the anaerobic reduction reaction mixture of hypocrellin (2) as compared to hypomycin A (4) in acetonitrile- $d_{3}$. 


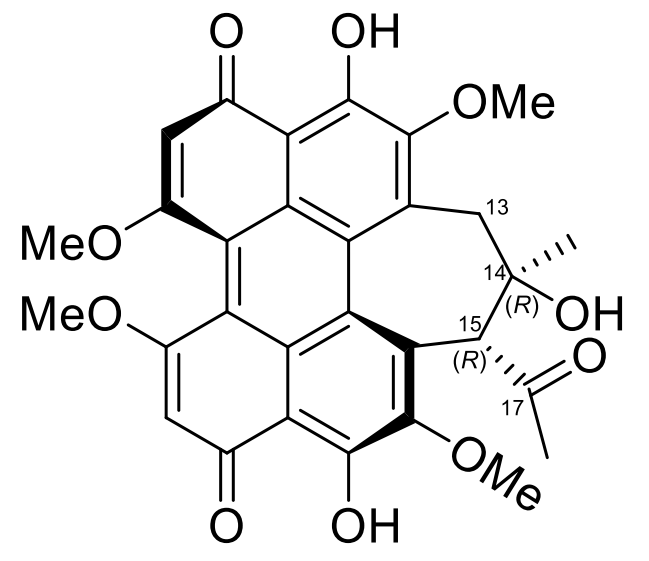

ent-Shiraiachrome A (1)



Hypomycin C (5)

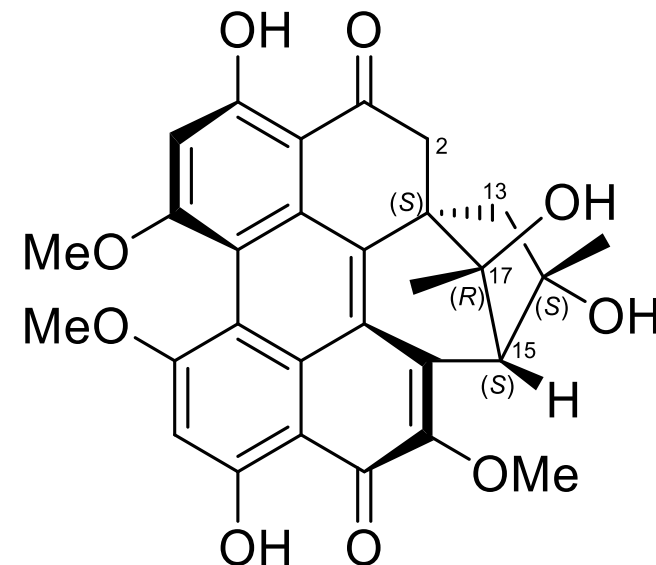

Hypomycin E (6)

Figure S37. Previously reported structures of hypomycin C (5) and E (6) as compared to ent-shiraiachrome A (1). ${ }^{6}$ 


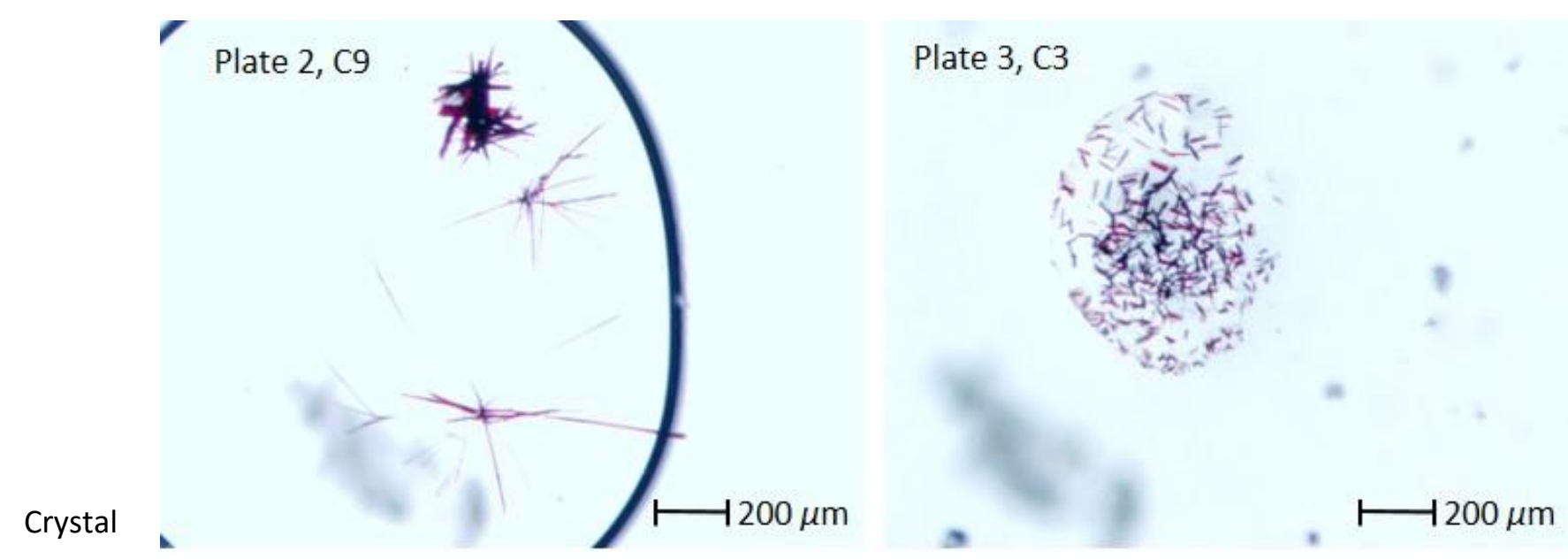

Figure S38. Typical microcrystals of ent-shiraiachrome A (1), prepared via ENaCt.

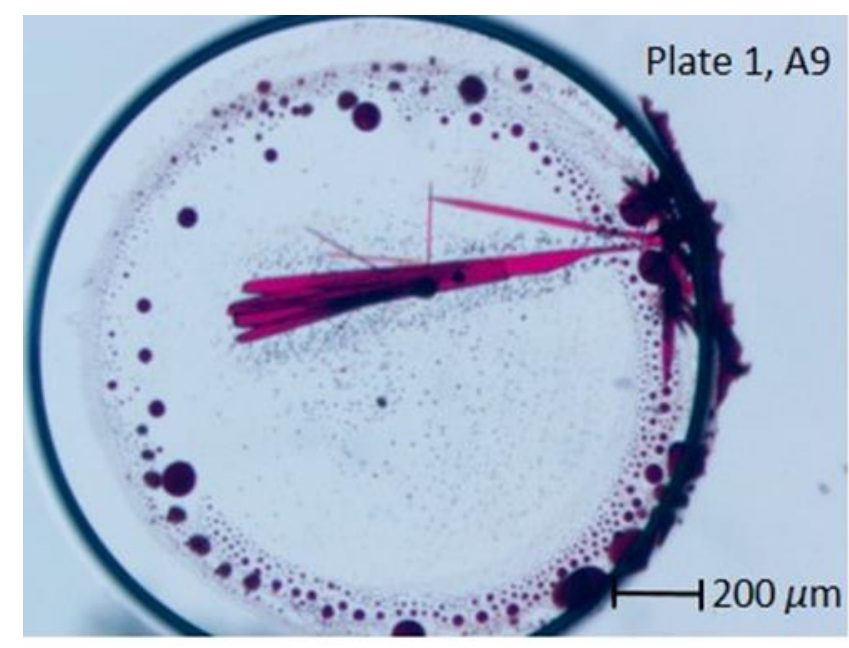

Figure S39. Single crystals of ent-shiraiachrome A (1) suitable for X-ray diffraction analysis, prepared via ENaCt after 14 days. 


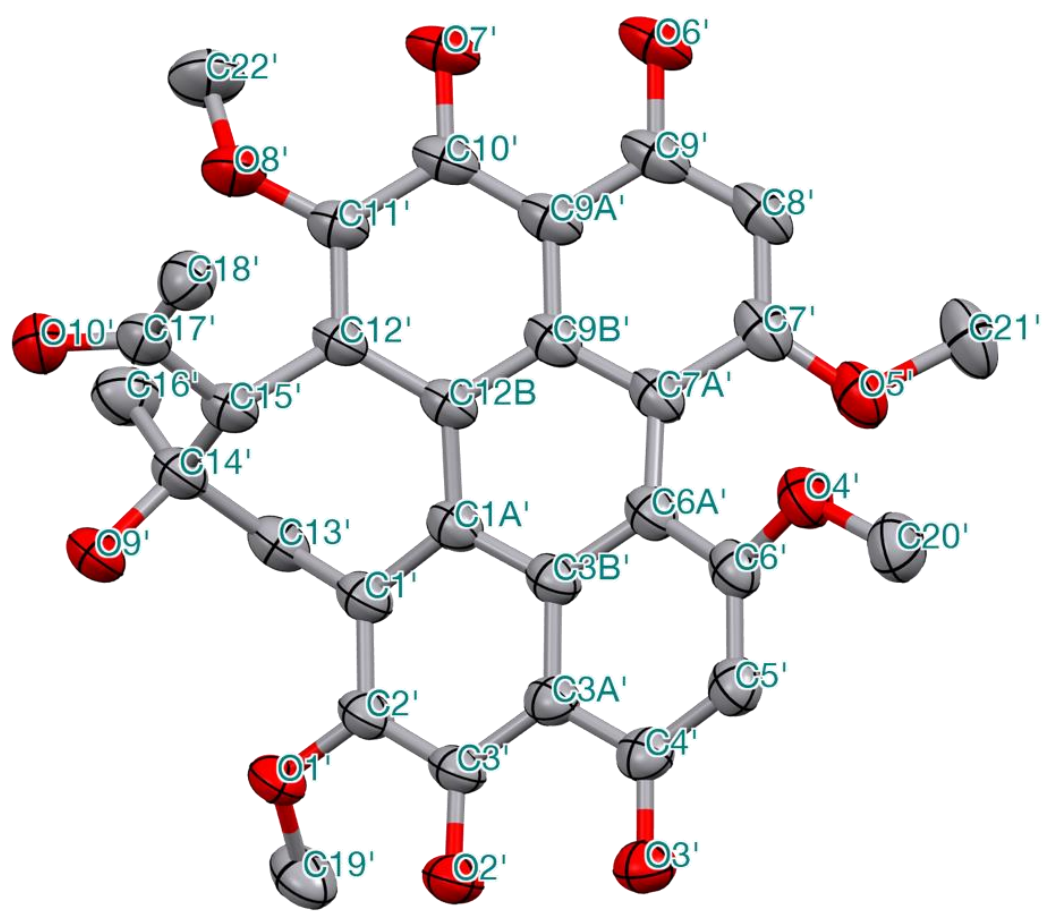

Figure S40. Displacement ellipsoid plot (50\% probability level) of ent-shiraiachrome A (1), showing the atom-labelling scheme. Hydrogen atoms have been omitted for clarity. Note that the two phenolic protons are located on $\mathrm{O}^{\prime}$ and $\mathrm{O}^{\prime}$ in the CIF file based on the difference density map while our HMBC data showed stronger association of these protons to $\mathrm{O}^{\prime}$ and $\mathrm{O}^{\prime}$. A close examination of the bond distances of ent-shiraiachrome A confirmed that these protons as well as the entire $\pi$-system are highly delocalized with a distribution of 60:40. ${ }^{1}$ The nature of the solvent, temperature, or other physical parameters can perturb the equilibrium in this system. 
Table S6. Crystal data and structure refinement for ent-shiraiachrome A (1).

\begin{tabular}{|c|c|}
\hline Identification code & klh_01082_45_2_p1_a9 \\
\hline Empirical formula & $\mathrm{C}_{30} \mathrm{H}_{26} \mathrm{O}_{10}$ \\
\hline Formula weight & 546.51 \\
\hline Temperature/K & 150.0 \\
\hline Crystal system & monoclinic \\
\hline Space group & $\mathrm{P} 2{ }_{1}$ \\
\hline $\mathrm{a} / \AA$ & $13.0485(5)$ \\
\hline $\mathrm{b} / \AA$ & $25.8680(10)$ \\
\hline $\mathrm{c} / \AA$ & $14.7103(6)$ \\
\hline$\alpha /^{\circ}$ & 90 \\
\hline$\beta /{ }^{\circ}$ & $116.105(2)$ \\
\hline$\gamma /{ }^{\circ}$ & 90 \\
\hline Volume $/ \AA^{3}$ & $4458.8(3)$ \\
\hline $\mathrm{Z}$ & 6 \\
\hline$\rho_{\text {calc }} \mathrm{g} / \mathrm{cm}^{3}$ & 1.221 \\
\hline$\mu / \mathrm{mm}^{-1}$ & 0.774 \\
\hline $\mathrm{F}(000)$ & 1716.0 \\
\hline Crystal size $/ \mathrm{mm}^{3}$ & $0.08 \times 0.03 \times 0.02$ \\
\hline Radiation & $\mathrm{CuK} \alpha(\lambda=1.54178)$ \\
\hline $2 \Theta$ range for data collection $/{ }^{\circ}$ & 6.692 to 136.722 \\
\hline Index ranges & $-14 \leq \mathrm{h} \leq 15,-31 \leq \mathrm{k} \leq 31,-17 \leq 1 \leq 17$ \\
\hline Reflections collected & 60509 \\
\hline Independent reflections & $16326\left[\mathrm{R}_{\mathrm{int}}=0.0378, \mathrm{R}_{\text {sigma }}=0.0292\right]$ \\
\hline Data/restraints/parameters & $16326 / 1069 / 1113$ \\
\hline Goodness-of-fit on $\mathrm{F}^{2}$ & 1.030 \\
\hline Final $R$ indexes $[\mathrm{I}>=2 \sigma(\mathrm{I})]$ & $\mathrm{R}_{1}=0.0360, \mathrm{w} \mathrm{R}_{2}=0.0931$ \\
\hline Final $\mathrm{R}$ indexes [all data] & $\mathrm{R}_{1}=0.0420, \mathrm{w} \mathrm{R}_{2}=0.0977$ \\
\hline Largest diff. peak/hole / e $\AA^{-3}$ & $0.12 /-0.15$ \\
\hline Flack parameter & $-0.01(7)$ \\
\hline
\end{tabular}




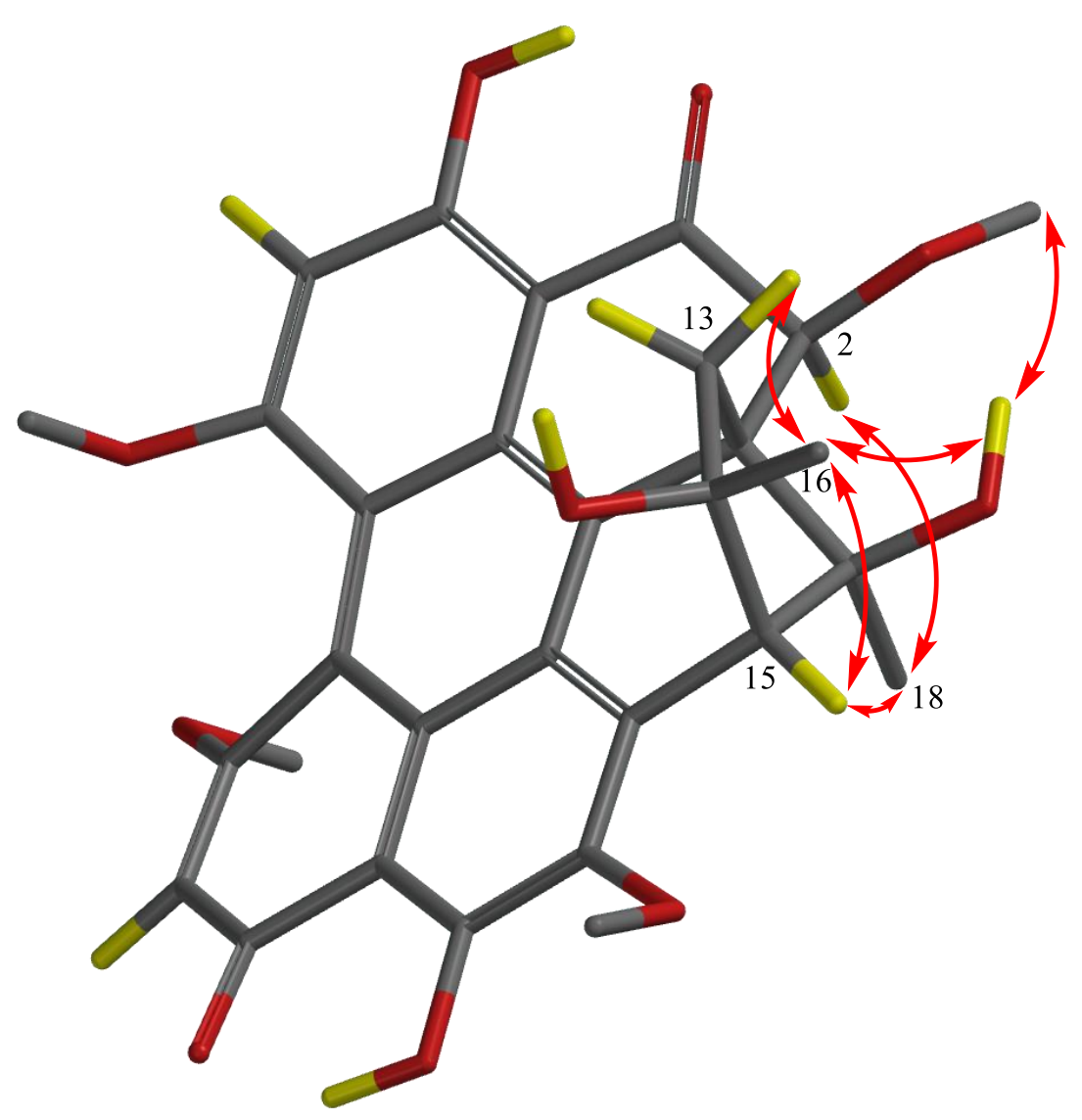

Hypomycin C (5)

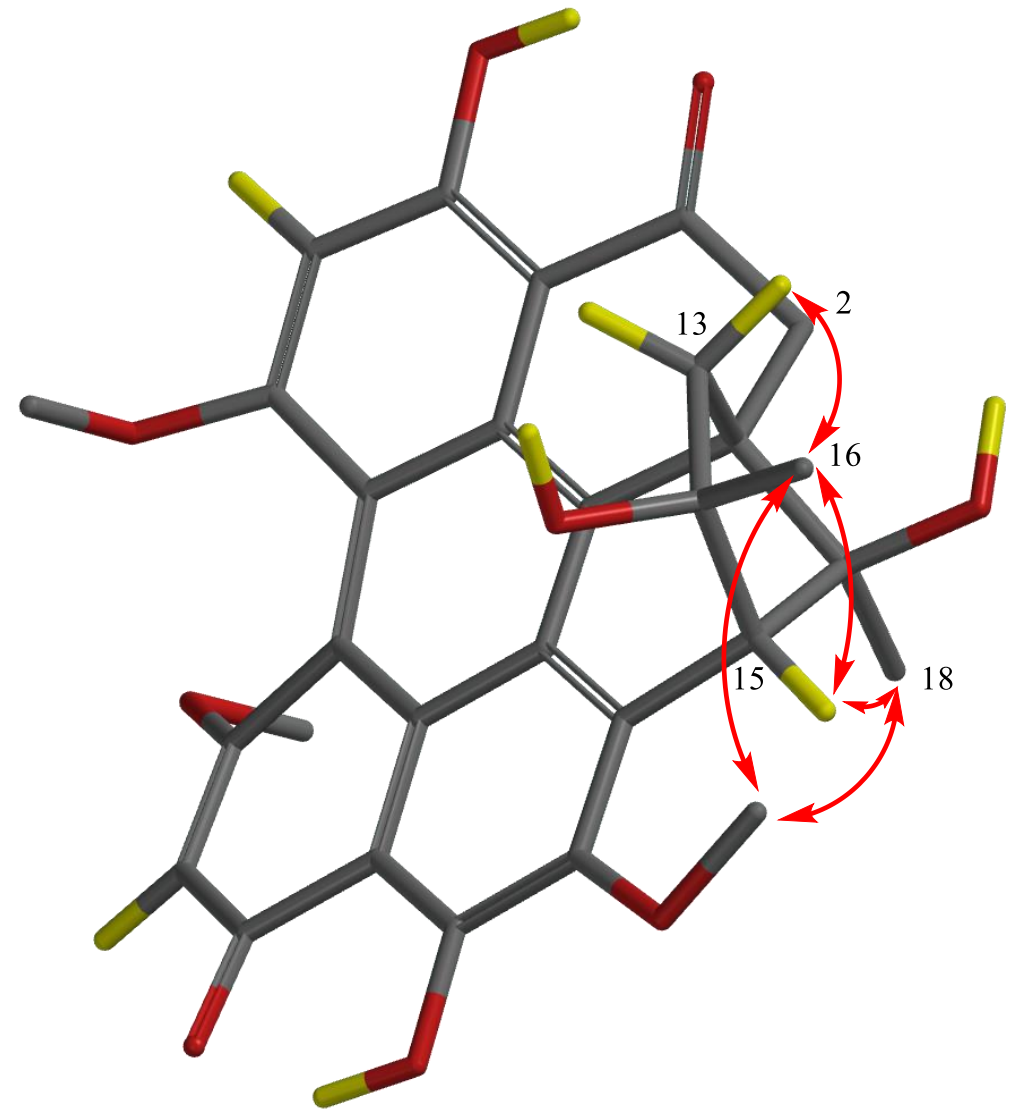

Hypomycin E (6)

Figure S41. Key NOESY correlations of hypomycin C (5) and hypomycin E (6). The 3D structures were constructed using Spartan'10 (version 1.1.0) to help visualize the NOESY correlations. 


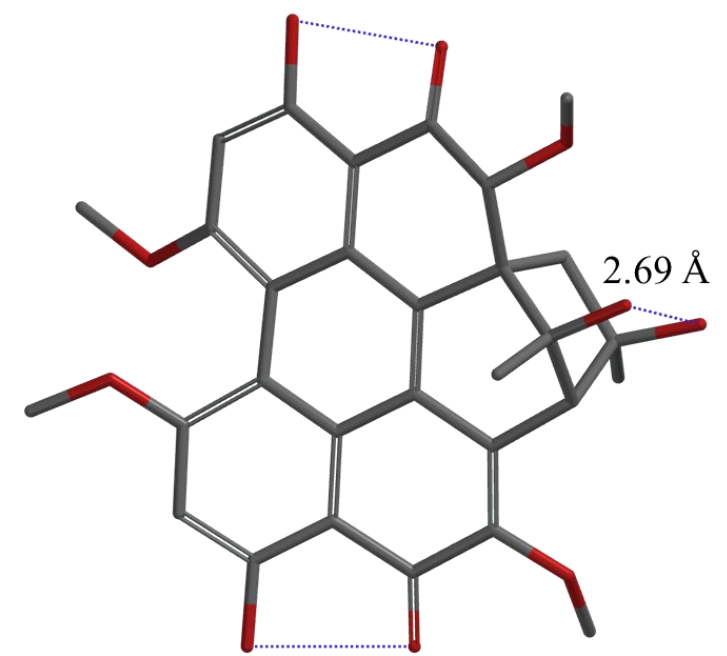

Hypomycin A (4)

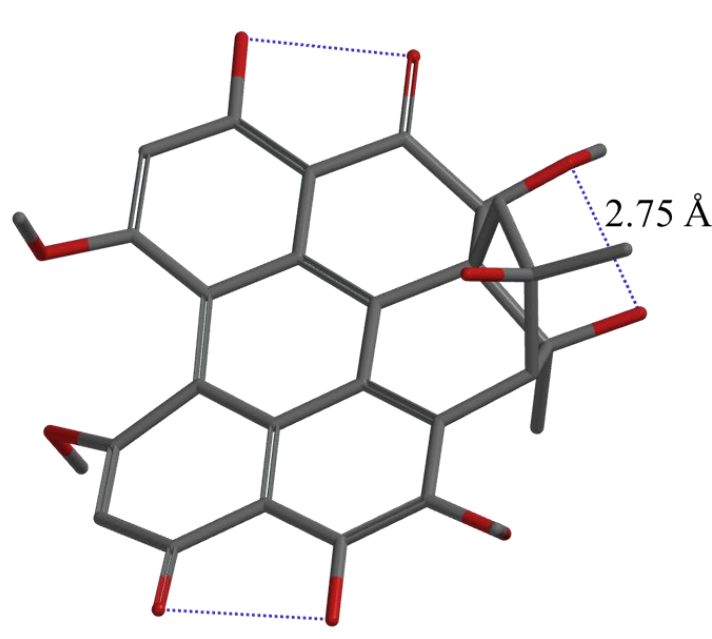

Hypomycin C (5)



Hypomycin E (6)

Figure S42. The observed intramolecular hydrogen-bonding of the exchangeable protons in hypomycin A (4), C (5), and E (6). The 3D molecular structures and donor-acceptor distances of the intramolecular hydrogen bonding interactions were obtained through Spartan'10 (version 1.1.0) similarity analysis. 


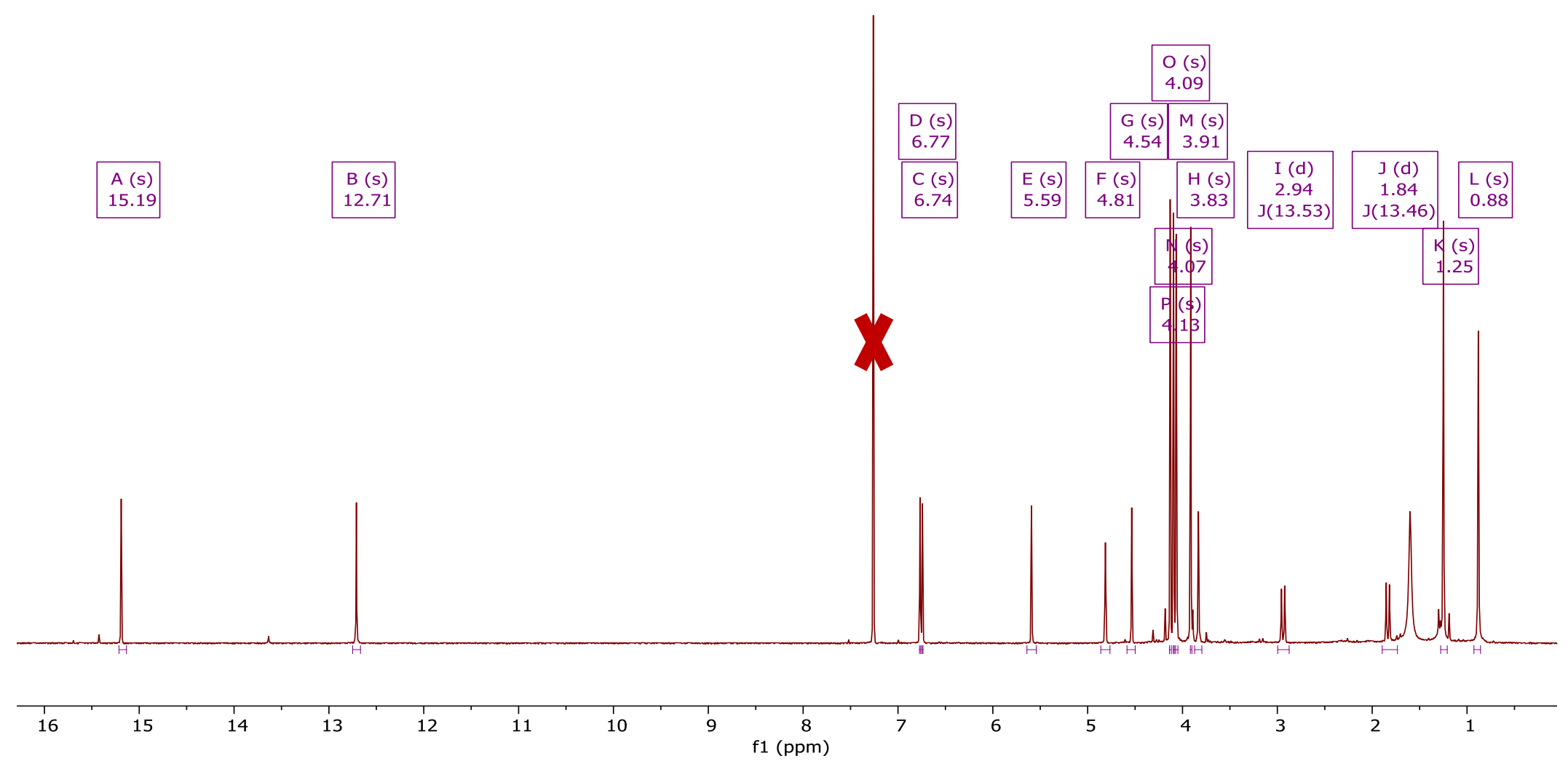

Figure S43. ${ }^{1} \mathrm{H}-\mathrm{NMR}$ of hypomycin A (4) $\left[400 \mathrm{MHz}, \mathrm{CDCl}_{3}\right]$. 


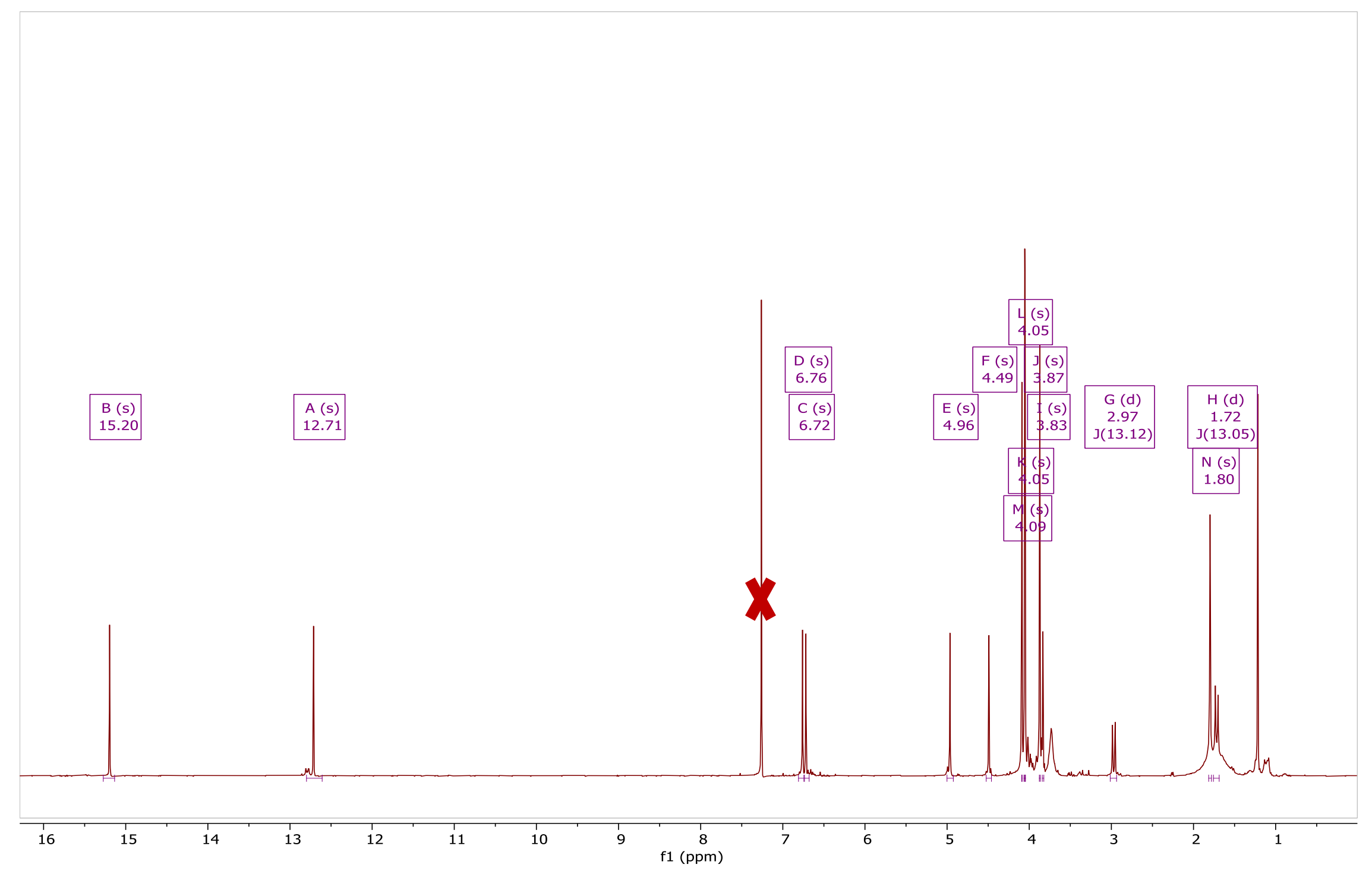

Figure S44. ${ }^{1} \mathrm{H}-\mathrm{NMR}$ of hypomycin C (5) [400 MHz, $\left.\mathrm{CDCl}_{3}\right]$. 


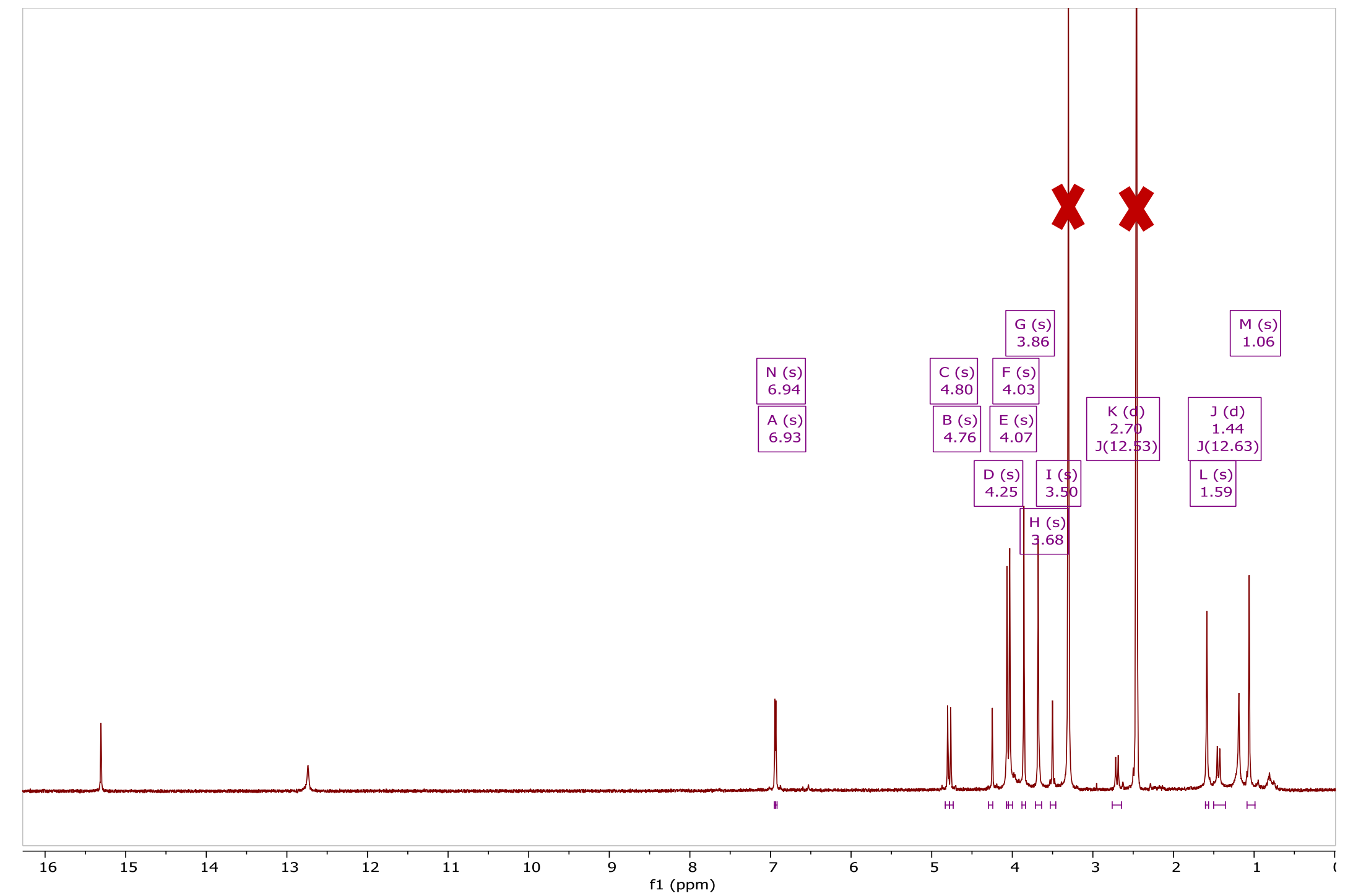

Figure S45. ${ }^{1} \mathrm{H}-\mathrm{NMR}$ of hypomycin C (5) $\left[400 \mathrm{MHz}, \mathrm{DMSO}-d_{6}\right]$. 


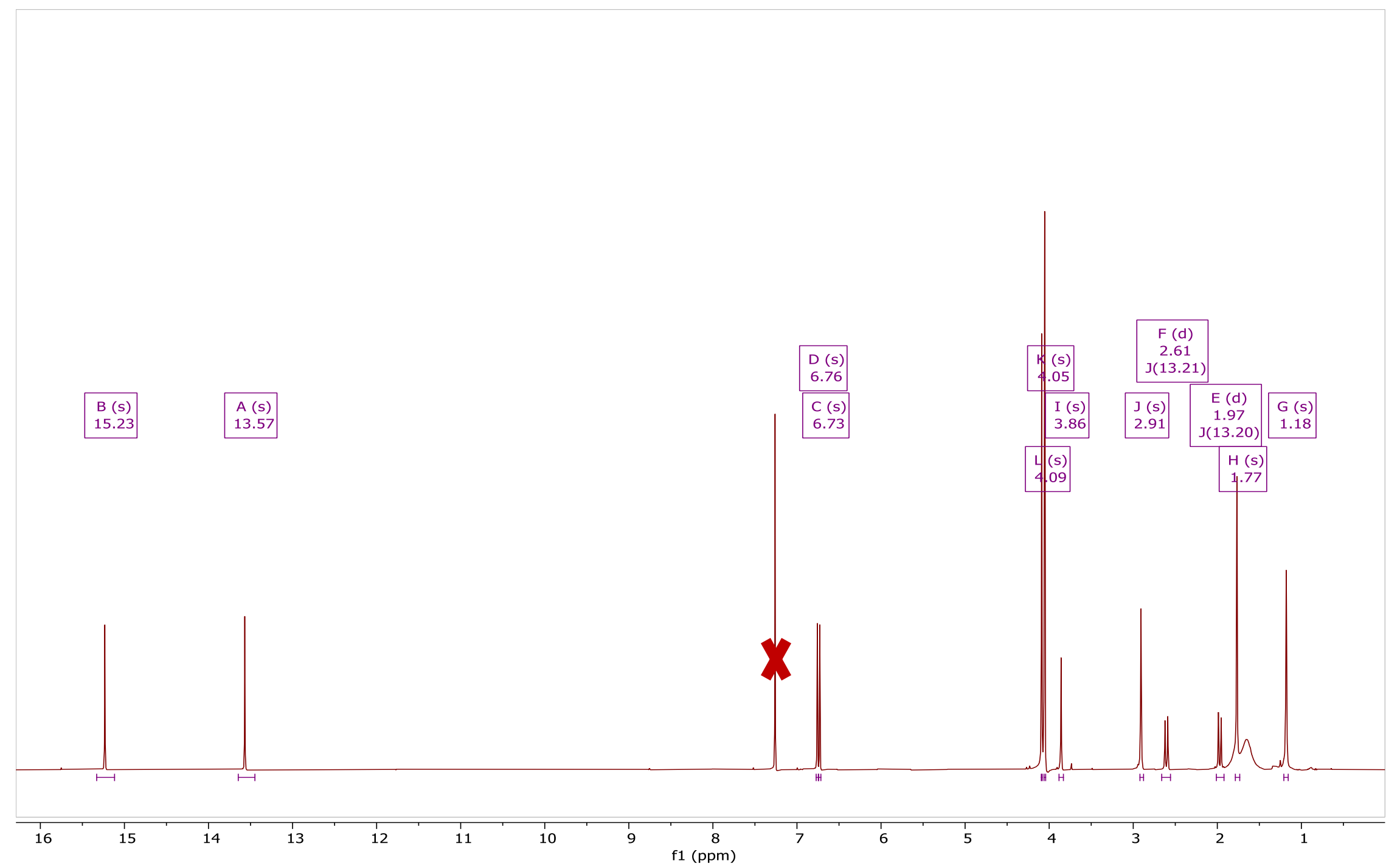

Figure S46. ${ }^{1} \mathrm{H}-\mathrm{NMR}$ of hypomycin E (6) [400 MHz, $\left.\mathrm{CDCl}_{3}\right]$. 
A
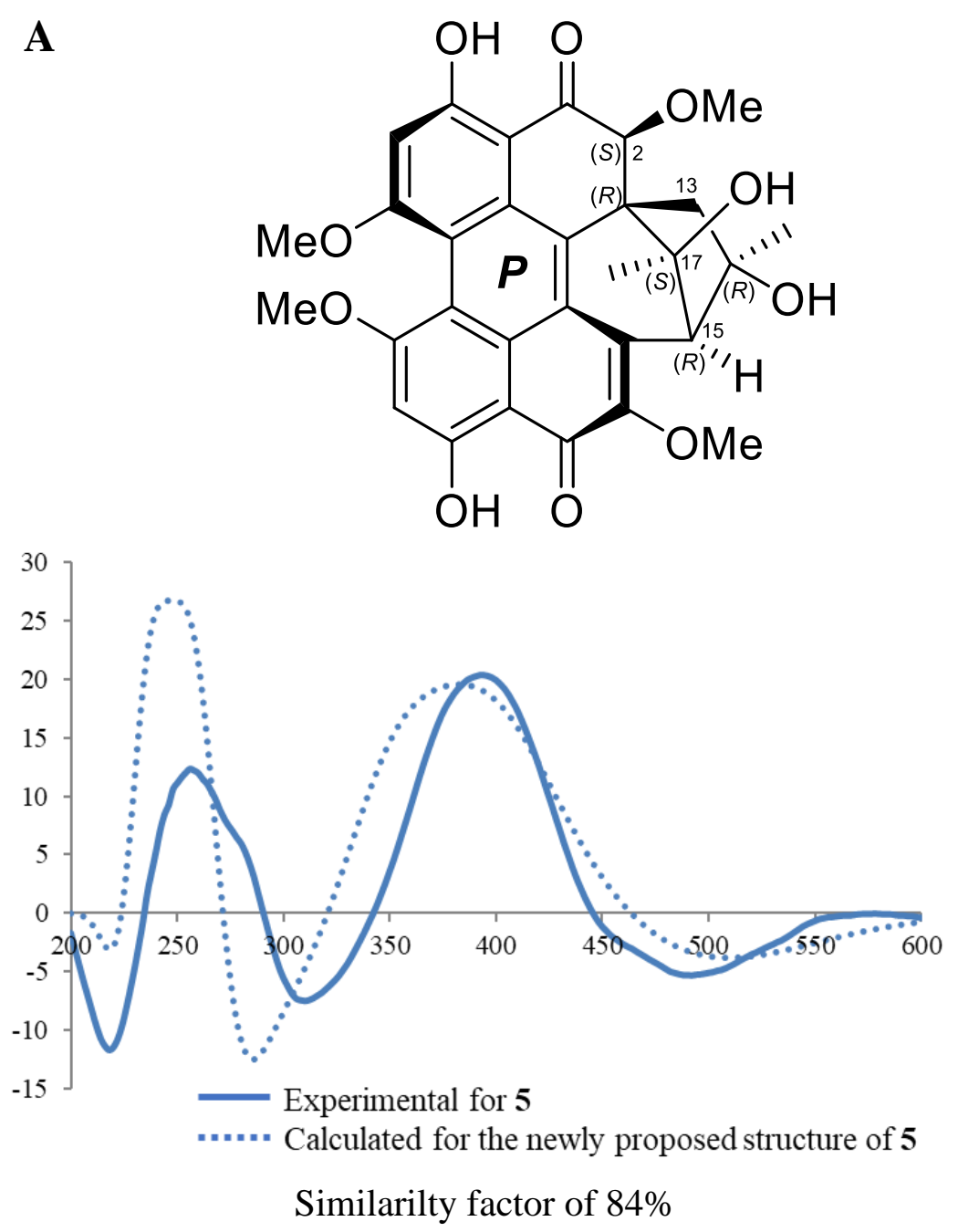

B

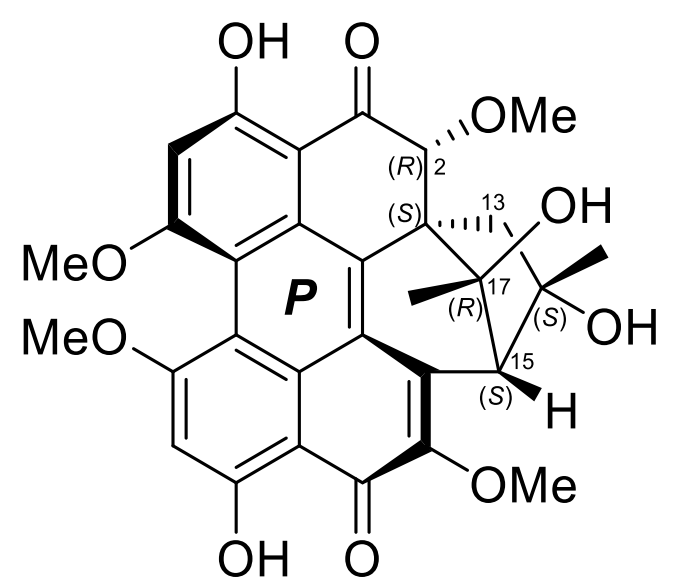



Figure S47. Experimental ECD spectrum of $\mathbf{5}$ compared with calculated ECD spectra of the newly-proposed configuration of $\mathbf{5}$ $[P(S), 1 R, 2 S, 14 R, 15 R, 17 S]$ on the left frame and the previously proposed configuration of $\mathbf{5}[P(S), 1 S, 2 R, 14 S, 15 S, 17 R]$ on the right frame. SpecDis software was utilized to calculate the similarity factors. 
Table S7. NMR Spectroscopic Data for 11-12 in $\mathrm{CDCl}_{3}(\delta$ in ppm).

\begin{tabular}{|c|c|c|c|c|}
\hline \multirow[b]{2}{*}{ position } & \multicolumn{2}{|r|}{$11^{\mathrm{a}}$} & \multicolumn{2}{|l|}{$12^{\mathrm{b}}$} \\
\hline & $\delta_{\mathrm{C}}$, type & $\delta_{\mathrm{H}}(J, \mathrm{~Hz})$ & $\delta_{\mathrm{C}}$, type & $\delta_{\mathrm{H}}(J, \mathrm{~Hz})$ \\
\hline 1 & 146.1, C & & $134.5, \mathrm{C}$ & \\
\hline $1 \mathrm{a}$ & $125.4, \mathrm{C}$ & & 124.3 or $126.8, \mathrm{C}$ & \\
\hline 2 & $125.5, \mathrm{CH}$ & $7.25, \mathrm{~s}$ & 151.0, C & \\
\hline 3 & $170.5, \mathrm{C}$ & & $165.8, \mathrm{C}$ & \\
\hline $3 a$ & $106.5, \mathrm{C}$ & & 107.6, C & \\
\hline $3 b$ & 123.6, C & & 124.3, C or $126.8, \mathrm{C}$ & \\
\hline 4 & $185.2, \mathrm{C}$ & & 185.3, C & \\
\hline 5 & 103.7, CH & $6.45, \mathrm{~s}$ & 103.7, $\mathrm{CH}$ or $103.8, \mathrm{CH}$ & $6.45, \mathrm{~s}$ \\
\hline 6 & 167.6, C & & 168.1, C & \\
\hline $6 a$ & $120.7, \mathrm{C}$ & & $121.9, \mathrm{C}$ & \\
\hline 7 & 168.3, C & & 167.9, C & \\
\hline $7 \mathrm{a}$ & $120.7, \mathrm{C}$ & & $120.8, \mathrm{C}$ & \\
\hline 8 & 103.2, $\mathrm{CH}$ & $6.48, \mathrm{~s}$ & 103.7, $\mathrm{CH}$ or $103.8, \mathrm{CH}$ & $6.43, \mathrm{~s}$ \\
\hline 9 & $184.2, \mathrm{C}$ & & 185.6, C & \\
\hline $9 \mathrm{a}$ & $107.8, \mathrm{C}$ & & 106.9, C & \\
\hline $9 b$ & 128.0, C & & 127.6, C & \\
\hline 10 & $166.8, \mathrm{C}$ & & 169.3, C & \\
\hline 11 & 148.9, C & & $122.3, \mathrm{CH}$ & $7.31, \mathrm{~s}$ \\
\hline 12 & $131.0, \mathrm{C}$ & & 134.0, C & \\
\hline $12 \mathrm{a}$ & $127.6, \mathrm{C}$ & & 126.0, C & \\
\hline 13 & $50.9, \mathrm{CH}_{2}$ & $2.78, \mathrm{~d}(14.0)$ & $43.3, \mathrm{CH}_{2}$ & 2.42, d (13.6) \\
\hline & & $3.00, \mathrm{~d}(14.0)$ & & $3.62, \mathrm{~d}(13.6)$ \\
\hline 14 & 79.4, C & & $80.5, \mathrm{C}$ & \\
\hline 15 & $63.9, \mathrm{CH}$ & $3.77, \mathrm{~s}$ & $66.1, \mathrm{CH}$ & $4.25, \mathrm{~s}$ \\
\hline 16 & $24.8, \mathrm{CH}_{3}$ & $1.77, \mathrm{~s}$ & $24.3, \mathrm{CH}_{3}$ & $1.77, \mathrm{~s}$ \\
\hline 17 & 206.8, C & & $207.4, \mathrm{C}$ & \\
\hline 18 & $28.6, \mathrm{CH}_{3}$ & $1.81, \mathrm{~s}$ & $32.2, \mathrm{CH}_{3}$ & $1.97, \mathrm{~s}$ \\
\hline $\mathrm{CH}_{3} \mathrm{O}-2$ & & & $61.9, \mathrm{CH}_{3}$ & $4.19, \mathrm{~s}$ \\
\hline $\mathrm{CH}_{3} \mathrm{O}-6$ & $56.5, \mathrm{CH}_{3}$ & $4.05, \mathrm{~s}$ & $56.6, \mathrm{CH}_{3}$ or $56.7, \mathrm{CH}_{3}$ & $4.06, \mathrm{~s}$ \\
\hline $\mathrm{CH}_{3} \mathrm{O}-7$ & $56.7, \mathrm{CH}_{3}$ & $4.07, \mathrm{~s}$ & $56.7, \mathrm{CH}_{3}$ or $56.6, \mathrm{CH}_{3}$ & $4.05, \mathrm{~s}$ \\
\hline $\mathrm{CH}_{3} \mathrm{O}-11$ & $61.3, \mathrm{CH}_{3}$ & $4.28, \mathrm{~s}$ & & \\
\hline $\mathrm{HO}-3$ & & $15.47, \mathrm{~s}$ & & \\
\hline HO-10 & & $16.10, \mathrm{~s}$ & & \\
\hline
\end{tabular}

${ }^{\text {a Recorded at }} 400 \mathrm{MHz}$ for ${ }^{1} \mathrm{H}$ and $100 \mathrm{MHz}$ for ${ }^{13} \mathrm{C}\left\{{ }^{1} \mathrm{H}\right\}$.

${ }^{\text {b}}$ Recorded at $700 \mathrm{MHz}$ for ${ }^{1} \mathrm{H}$ and $175 \mathrm{MHz}$ for ${ }^{13} \mathrm{C}\left\{{ }^{1} \mathrm{H}\right\}$. 

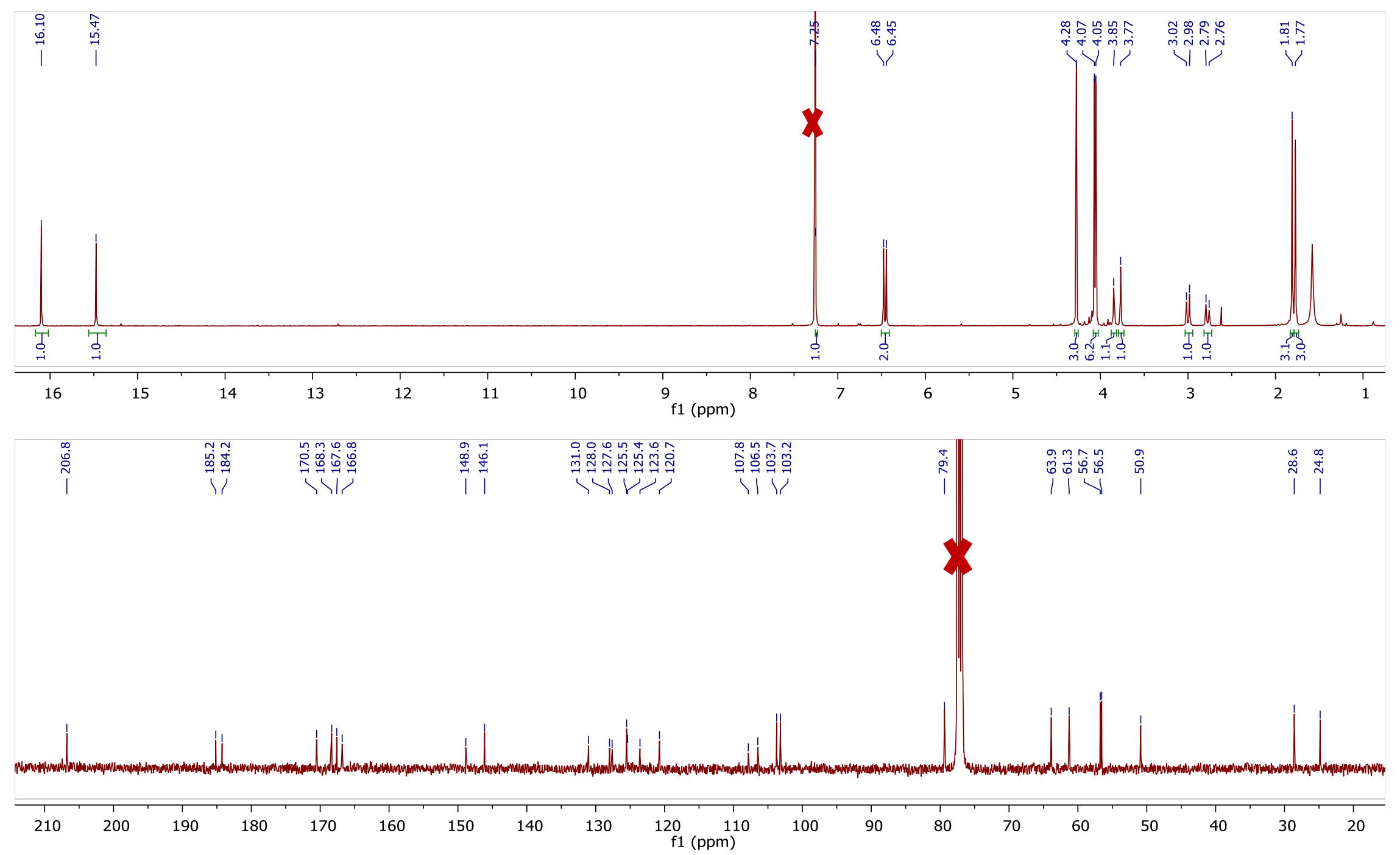

Figure S48. ${ }^{1} \mathrm{H}$ - and ${ }^{13} \mathrm{C}\left\{{ }^{1} \mathrm{H}\right\}$-NMR spectra of $\mathbf{1 1}\left[400 \mathrm{MHz}\right.$ for ${ }^{1} \mathrm{H}$ and $100 \mathrm{MHz}$ for $\left.{ }^{13} \mathrm{C}\left\{{ }^{1} \mathrm{H}\right\}, \mathrm{CDCl}_{3}\right]$. 

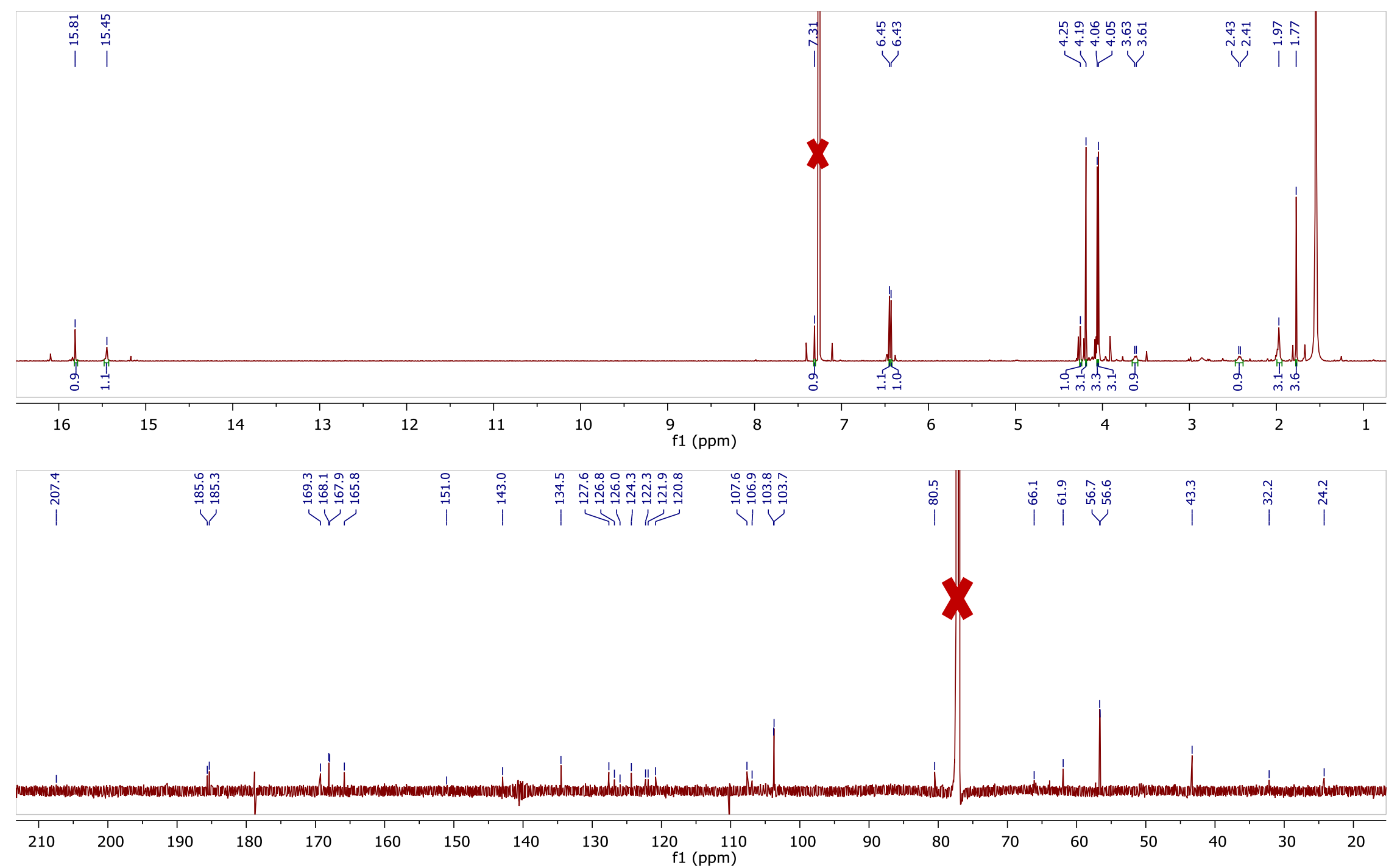

Figure S49. ${ }^{1} \mathrm{H}$ - and ${ }^{13} \mathrm{C}\left\{{ }^{1} \mathrm{H}\right\}$-NMR spectra of $12\left[700 \mathrm{MHz}\right.$ for ${ }^{1} \mathrm{H}$ and $175 \mathrm{MHz}$ for $\left.{ }^{13} \mathrm{C}\left\{{ }^{1} \mathrm{H}\right\}, \mathrm{CDCl}_{3}\right]$. 


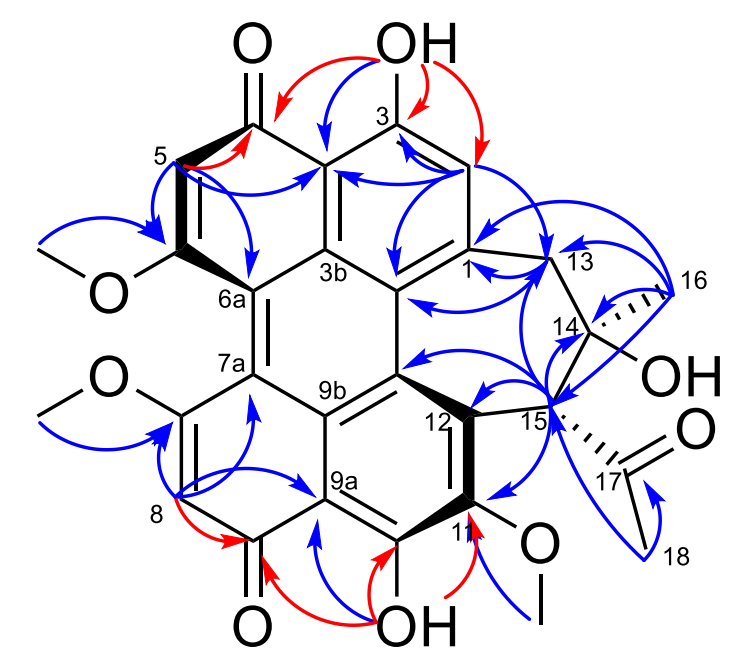

11

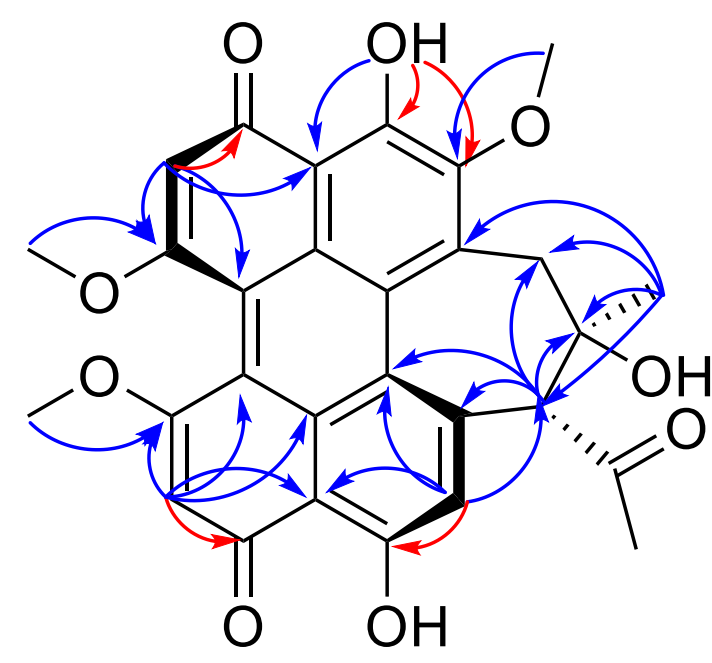

12

Figure S50. Key HMBC correlations for compounds 11 and 12. Arrows highlighted in red confirm the tautomeric structures of $\mathbf{1 1}$ and $\mathbf{1 2}$. 


\section{References}

1. T. Steiner. The Hydrogen Bond in the Solid State. Angew. Chem. Int. Ed. Engl. 2002, 41, 49-76. 